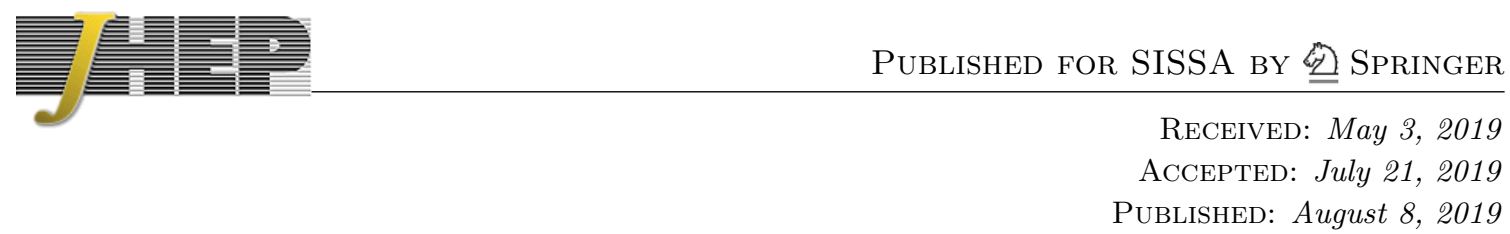

\title{
Symmetries near the horizon
}

\author{
Henry W. Lin, ${ }^{a}$ Juan Maldacena ${ }^{b}$ and Ying Zhao ${ }^{b}$ \\ ${ }^{a}$ Physics Department, Princeton University, \\ Jadwin Hall, Washington Road, Princeton, NJ 08540, U.S.A. \\ ${ }^{b}$ School of Natural Sciences, Institute for Advanced Study, \\ 1 Einstein Drive, Princeton, NJ 08540, U.S.A. \\ E-mail: hwlin@princeton.edu, malda@ias.edu, zhaoying@ias.edu
}

ABSTRACT: We consider a nearly- $\mathrm{AdS}_{2}$ gravity theory on the two-sided wormhole geometry. We construct three gauge-invariant operators in $\mathrm{NAdS}_{2}$ which move bulk matter relative to the dynamical boundaries. In a two-sided system, these operators satisfy an $\operatorname{SL}(2)$ algebra (up to non perturbative corrections). In a semiclassical limit, these generators act like SL(2) transformations of the boundary time, or conformal symmetries of the two sided boundary theory. These can be used to define an operator-state mapping. A particular large $N$ and low temperature limit of the SYK model has precisely the same structure, and this construction of the exact generators also applies. We also discuss approximate, but simpler, constructions of the generators in the SYK model. These are closely related to the "size" operator and are connected to the maximal chaos behavior captured by out of time order correlators.

KEYwords: AdS-CFT Correspondence, Black Holes, Conformal Field Theory

ArXiv EPRINT: 1904.12820 


\section{Contents}

1 Introduction and motivation 1

2 Review 3

2.1 Review of the symmetries of $A d S_{2}$

2.2 Review of the nearly- $A d S_{2}$ gravity theory 6

2.3 Review of SYK 8

3 Exact generators $\quad 9$

3.1 Construction of gauge invariant SL(2) generators 9

3.1.1 Writing the charges purely in terms of boundary quantities 11

$\begin{array}{ll}3.2 & \text { Defining "conserved" charges } \\ \end{array}$

4 Approximate expressions for the generators 13

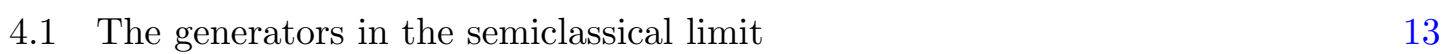

4.2 The semiclassical limit and SL(2) symmetries of the physical boundary time 15

$\begin{array}{lll}\text { 4.2.1 Inserting matter at early lorentzian times } & 19\end{array}$

4.2.2 Inserting matter beyond the scrambling time and corrections to the semiclassical limit $\quad 20$

4.3 Other semiclassical expressions for the generators 21

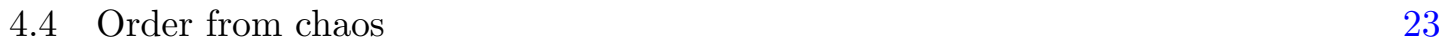

$\begin{array}{ll}4.5 & \text { Generators for the one-sided case } 25\end{array}$

5 Exploring the bulk $\quad 28$

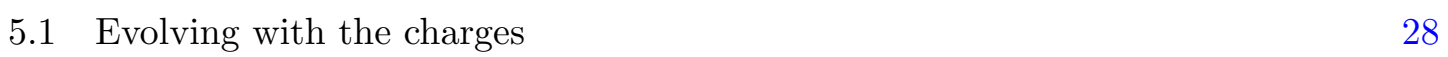

5.2 Exploring behind the horizon or moving the horizon 30

5.3 The inner horizon or Cauchy horizon 31

5.4 Moving operators into the bulk 32

6 Connection to SYK and other systems $\quad 34$

6.1 Generators in SYK 34

6.2 Relation to "size" 36

6.3 Analogy to the Rindler (and AdS-Rindler) decomposition of a higher dimen$\begin{array}{ll}\text { sional field theory } & 39\end{array}$

$\begin{array}{lll}7 & \text { Discussion } & 40\end{array}$

$\begin{array}{lll}7.1 & \text { Measuring distance } & 40\end{array}$

$\begin{array}{lll}7.2 & \text { Conclusions and open questions } & 42\end{array}$

$\begin{array}{ll}\text { A SO(3) analogy } & 44\end{array}$

A.1 Exact $\mathrm{SO}(3)$ algebra from two copies $\quad 45$

A.2 Action on states/operators 46 
D Spinor description of the boundary variables 49

D.1 Computation of the charges beyond the scrambling time 49

E OTOC correlators and the expectation values of charges $\quad 50$

$\begin{array}{lll}\text { F Stiffer traversable wormholes } & 51\end{array}$

G Measuring distance using a single free field 53

\section{Introduction and motivation}

Any black hole with finite temperature has a near horizon geometry that can be approximated by flat space. The boost symmetry of this flat space region corresponds to the full modular Hamiltonian of the outside region of the black hole, and it is an exact symmetry of the full wormhole geometry. The two translation symmetries of this flat space region are more mysterious. It is important to understand them because they can take matter into the black hole interior. In this paper, we construct explicitly these symmetries for nearly $\mathrm{AdS}_{2}$ gravity and also for the related SYK model.

Nearly $\mathrm{AdS}_{2}\left(\mathrm{NAdS}_{2}\right)$ gravity [1-4] captures the gravitational dynamics of near extremal black holes after a Kaluza-Klein reduction. The important gravitational mode is non-propagating and can be viewed as living at the boundaries of the nearly $\mathrm{AdS}_{2}$ region. The action of these boundary modes is universal and can be written in terms of a Schwarzian action for a variable that can be viewed as a map from the boundary proper time to a time coordinate in a rigid $\mathrm{AdS}_{2}$ spacetime. A similar mode appears in the description of Nearly $\mathrm{CFT}_{1}\left(\mathrm{NCFT}_{1}\right)$ quantum systems, such as the SYK model [5-9], which exhibit nearly conformally-invariant correlation functions at relatively low energies.

In these systems, there is an approximation where matter appears to move in a rigid $\mathrm{AdS}_{2}$ background geometry displaying an $\widetilde{\mathrm{SL}}(2, \mathbb{R})$ isometry group ${ }^{1}$ henceforth denoted by $\mathrm{SL}(2)$. This approximation becomes better and better as the boundaries are further and further away. However, this does not obviously translate into a physical symmetry, since only the relative position between the boundaries and the bulk matter is physical. Nevertheless, we will find three SL(2) generators that act on the full physical Hilbert space of the system. These generators obey an exact SL(2) algebra, but they do not commute with the Hamiltonian. However, they have a relatively simple behavior under Hamiltonian evolution, which can be used to define "conserved" charges through a more subtle construction.

\footnotetext{
${ }^{1}$ In Euclidean signature, the isometry group is $\operatorname{PSL}(2, \mathbb{R})$. In this paper, we are mostly concerned about the algebra and not the group.
} 
It is convenient to describe the $\mathrm{NAdS}_{2} / \mathrm{NCFT}_{1}$ system in terms of an extended Hilbert space with a gauge constraint. The extended Hilbert space factorizes into three pieces: two systems describing the two boundaries of the eternal black hole and a system describing the bulk matter fields. Each of them can be viewed in terms of particles moving on an exact $\mathrm{AdS}_{2}$ spacetime [10,11]. The physical Hilbert space is obtained by imposing an $\mathrm{SL}(2)_{g}$ gauge constraint that sets to zero the overall $\mathrm{SL}(2)_{g}$ charges of the three systems. This constraint imposes that only the relative position between the two boundaries, or between the boundary and the bulk matter, are physical. In this paper we discuss physical $\mathrm{SL}(2)$ generators which are invariant under the $\mathrm{SL}(2)_{g}$ gauge symmetry. It is important not to confuse these two SL(2) groups, the gauge one and the physical one. This paper is about they physical one. Our construction will define these physical generators relative to boundary positions in such a way that they are invariant under the gauge symmetries. Due to the fact that they involve the boundary positions, they are not conserved under time evolution, since the boundary positions change in time. However, the dynamics of the boundary positions is integrable [10-15], and one could use this fact to define "conserved" charges by simply "undoing" the boundary evolution.

Our discussion is exact in a scaling limit where we go to low temperatures, but we scale up the size of the black hole so that we keep fixed the coupling of the Schwarzian mode, or the quantum gravitational effects in $\mathrm{AdS}_{2}$. This is a limit where the near extremal entropy $\Delta S=S-S_{e}$ is kept fixed. ${ }^{2}$ In SYK variables this is the limit $N \rightarrow \infty, \beta J \rightarrow \infty$ with $N /(\beta J)$ fixed. We have not included finite $\beta J$ effects or finite $N$ effects. The generators we define involve variables, such as the distance between the two boundaries, which are well defined in the gravity theory, in the scaling limit we define, but are not expected to make sense when non-perturbative effects are taken into account. In particular, they are not expected to make sense for finite $N$ in the SYK model. This should not be surprising since unitary SL(2) representations are infinite dimensional. However, we also relate the generators we defined to other operators which are well defined for finite $N$, but agree with the generators in the semiclassical limit. This allows us to identify operators in both a gravity theory and the SYK model which approximately obey an SL(2) algebra and should be identified with the symmetries of $\mathrm{AdS}_{2}$. These approximate symmetries behave as $\mathrm{SL}(2)_{u}$ transformations of the physical boundary time of a pair of $\mathrm{NCFT}_{1} \mathrm{~s}$, and we give an approximate state-operator map that organizes the $\mathrm{NCFT}_{1}$ Hilbert space into primaries and descendants, in analogy with higher dimensions.

These generators are connected to the operators that generate traversable wormholes [16]. These move matter from one side of the horizon to the other. In fact, the approximate expression for the global time translation operator is essentially the same as the coupled Hamiltonian in [17]. These approximate generators also make contact with another approach for describing bulk motion via the "size" operator in [18-20]. So the discussion in this paper explains why such operators act like approximate $\mathrm{SL}(2)$ isometries in the bulk. We also point out that the structure of the approximate generators is similar

\footnotetext{
${ }^{2}$ For a four dimensional charged near extremal black hole this is $\Delta S \propto r_{e}^{3} T / l_{p}^{2}$, where $r_{e}$ is the extremal radius. We take $\Delta S$ fixed with $r_{s} \rightarrow \infty, T \rightarrow 0$.
} 
to the structure of the exact generators in the case of higher dimensional conformal field theories in Rindler space.

Outline. In section two, we review nearly $\mathrm{AdS}_{2}$ gravity at low energies in the embedding space formalism. The Hilbert space of the system consists of two boundary modes plus arbitrary matter, with an overall $\mathrm{SL}(2)$ gauge constraint. We briefly review how this structure also emerges in the SYK model.

In section three, we construct the generators which satisfy an exact $\mathrm{SL}(2)$ algebra. Although the quadratic Casimir commutes with the usual Hamiltonians $H_{l}$ or $H_{r}$, the individual generators do not. We nevertheless explain how to obtain conserved charges.

In section four, we consider the charges in the semi-classical limit. In this limit, the generators can be viewed as conformal symmetries of the boundary time. We show that the Hilbert space organizes into primaries and descendants and give a state-operator correspondence analogous to the higher dimensional versions.

In section five, we show how to use these charges to explore bulk physics. We comment on drama near the inner horizon. We also discuss applications of our construction to previous work. Our charges are closely related to the coupled Hamiltonian in [17].

In section six, we explain how these approximate charges can be realized in the SYK model. We also relate our charges to "size" in SYK [19-21]. We note that the generators written in terms of the microscopic variables has an analogous form in higher dimensional CFTs.

In section seven, we discuss some issues and draw conclusions.

In the appendices, we explain how to construct gauge-invariant $\mathrm{SO}(3)$ generators in a rather pedestrian system involving two non-relativistic particles on a sphere, plus some arbitrary matter, with an overall angular momentum gauge constraint. We also explain how to compute commutators in both the canonically quantized Schwarzian theory and its linearized cousin. We also discuss an alternative to the embedding space formalism which uses SL(2) spinors instead of the vectors. Finally, we comment on a modified eternal traversable wormhole where the oscillation frequency of the Schwarzian mode is very large.

Notation. In most of this paper we work in units, where in the SYK language $\alpha_{s} N / \mathcal{J}=$ 1 , or in $4 \mathrm{~d}$ near extremal charge black hole language, $\frac{r_{e}^{3}}{G_{N}}=1$, where $r_{e}$ is the extremal radius. Such factors can be restored by dimensional analysis.

\section{Review}

\subsection{Review of the symmetries of $A d S_{2}$}

In the embedding space formalism, $\mathrm{AdS}_{2}$ is the universal cover of the surface defined by

$$
Y . Y=\eta_{a b} Y^{a} Y^{b}=-\left(Y^{-1}\right)^{2}-\left(Y^{0}\right)^{2}+\left(Y^{1}\right)^{2}=-1 .
$$

From this definition, it is clear that $\mathrm{AdS}_{2}$ has an $\mathrm{SO}(2,1) \simeq \mathrm{SL}(2, R)$ symmetry generated by

$$
Q^{a}=\frac{1}{2} \epsilon^{a b c} J_{b c}, \quad J_{a b}=-i Y_{a} \frac{\partial}{\partial Y^{b}}+i Y_{b} \frac{\partial}{\partial Y^{a}}
$$



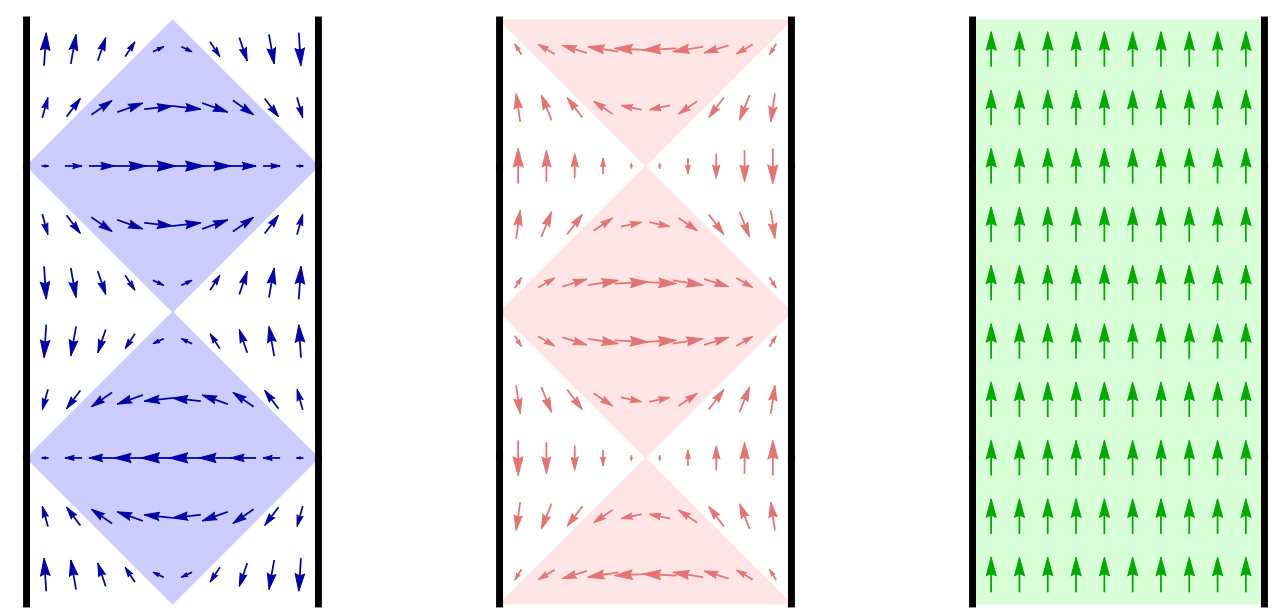

Figure 1. The three Killing vectors: boost $B$ (blue), momentum $P$ (pink), and global energy $E$ (green) given in (2.5) in the coordinate system (2.6a). The shaded regions delineate different orbits of the symmetries.

These generators satisfy the algebra

$$
\left[Q^{a}, Q^{b}\right]=i \epsilon^{a b c} \eta_{c d} Q^{d}
$$

To see how these generators act on $\mathrm{AdS}_{2}$ more explicitly, we can solve the constraint 2.1 using global coordinates:

$$
Y=\left(Y^{-1}, Y^{0}, Y^{1}\right)=\left(\frac{\cos T}{\sin \sigma}, \frac{\sin T}{\sin \sigma}, \frac{-1}{\tan \sigma}\right), \quad \sigma \in[0, \pi] .
$$

Then, the Killing vectors

$$
\begin{aligned}
& B=Q^{-1}=J_{0,1}=i\left(-\cos T \cos \sigma \partial_{T}+\sin T \sin \sigma \partial_{\sigma}\right) \\
& P=Q^{0}=-J_{-1,1}=-i\left(\sin T \cos \sigma \partial_{T}+\cos T \sin \sigma \partial_{\sigma}\right) \\
& E=Q^{1}=J_{-1,0}=i \partial_{T}
\end{aligned}
$$

Note that $P=-i[B, E]$. Near the bifurcation point $T=0, \sigma=\pi / 2$ these symmetries act as time translation/energy $E$, spatial translation/momentum $P$, and boost $B$. See figure 1 . Of course, the algebra is $\mathrm{SL}(2)$, not Poincare, so that $[E, P]=i B$.

We can choose coordinate systems ${ }^{3}$ that simplify the action of these generators

$$
\begin{aligned}
& \text { Rindler : } \quad d s^{2}=-d t^{2} \sinh ^{2} \rho+d \rho^{2}, \quad \rho \in[-\infty, \infty], \quad B=i \partial_{t} \\
& \text { FLRW : } \quad d s^{2}=-d \tau^{2}+\sin ^{2} \tau d x^{2}, \quad \tau \in[0, \pi], \quad P=-i \partial_{x} \\
& \text { Global : } \quad d s^{2}=\frac{-d T^{2}+d \sigma^{2}}{\sin ^{2} \sigma}, \quad \sigma \in[0, \pi], \quad E=i \partial_{T}
\end{aligned}
$$

\footnotetext{
${ }^{3}$ The second coordinates describe a Friedmann-Lemaitre-Robertson-Walker (FLRW) cosmology.
} 
Notice that, given a vector $W^{a}$, we can assign a charge $Q_{W}=W_{a} Q^{a}$. This charge has the property that it leaves the bulk point $Y^{a} \propto W^{a}$ fixed. ${ }^{4}$ This is basically just the familiar fact that a rotation about some axis fixes the axis.

Points at the boundary are naturally described in terms of projective coordinates, $\tilde{X}^{a}$ with the constraint $\tilde{X} . \tilde{X}=0$ and the identification $\tilde{X}^{a} \sim \lambda \tilde{X}^{a}$. If we have a charge associated to the vector $W^{a}, Q_{W}=W \cdot Q$, then this charge will leave invariant the boundary points that are light-like separated from $W^{a}, W \cdot \tilde{X}=0$.

A particle moving in $\mathrm{AdS}_{2}$ can be described by a trajectory $Y^{a}(u)$ constrained to live on the surface $Y . Y=-1$. Since $Y$ is a vector, $\left[Q^{a}, Y^{b}\right]=i \epsilon^{a b c} Y_{c}$. For a standard massive particle, of mass $m$, the charges are given by

$$
Q^{a}=m \epsilon_{b c}^{a} Y^{b} \dot{Y}^{c}, \quad Y . Y=-1, \quad \dot{Y} . \dot{Y}=-1
$$

If the particle is also charged under an electric field that is uniform in $A d S_{2}$, then the charges are

$$
Q^{a}=m \epsilon_{b c}^{a} Y^{b} \dot{Y}^{c}-\mathfrak{q} Y^{a}, \quad Y . Y=-1, \quad \dot{Y} \cdot \dot{Y}=-1
$$

The charges $Q^{a}$ are conserved, and the particle trajectories are given by $Q . Y=\mathfrak{q}$.

Alternatively we can say that if we have a particle moving in $A d S_{2}$, its Hilbert space has operators satsifying

$$
\begin{aligned}
& {\left[Y^{a}, Y^{b}\right]=0,} \\
& {\left[Q^{a}, Q^{b}\right]=i \epsilon^{a b c} \eta_{c d} Q^{d},} \\
& {\left[Q^{a}, Y^{b}\right]=i \epsilon^{a b c} \eta_{c d} Y^{d} .}
\end{aligned}
$$

This is the Poincare algebra $R^{2,1} \rtimes \mathrm{SL}(2, R)$. The Casimirs of this algebra are

$$
r^{2}=Y . Y, \quad \mathfrak{q}=Y \cdot Q
$$

The values of these Casimirs are inputs of the physical theory. For example, for a spin-less particle freely propagating in $\mathrm{AdS}_{2}$ we have $r^{2}=-1, \mathfrak{q}=0$. From this point of view $\mathfrak{q}$ is the spin of the particle.

If we have quantum fields moving on $A d S_{2}$ the charges can be written in terms of the stress tensor and the associated Killing vector

$$
Q_{\zeta}=\int_{\Sigma} n^{\mu} T_{\mu \nu} \zeta^{\nu}
$$

where $\zeta^{\mu}$ are each of the Killing vectors in (2.5). These charges are constant and independent of the spatial slice $\Sigma$ used to evaluate them, if the fields obey appropriate reflecting conditions at the $\mathrm{AdS}_{2}$ boundary.

\footnotetext{
${ }^{4}$ If the vector $W^{a}$ is spacelike, then we will not have any fixed point in $A d S_{2}$. An example is the generator $E$ in (2.5), see figure 1.
} 


\subsection{Review of the nearly- $A d S_{2}$ gravity theory}

We will be considering the JT theory coupled to matter as [22-25]

$$
S=\phi_{0}\left[\int R-2 \int K\right]+\int \phi(R+2)-2 \phi_{b} \int K+S_{m}\left[g_{\mu \nu}, \chi\right]
$$

where we have also indicated the boundary terms. The first term is topological and only contributes to the extremal entropy. We have also assumed that the matter couples to the metric but not to $\phi$. We will also assume that the boundary is very far away so that matter effectively feels as if it was in exactly $A d S_{2}$ space. This is sometimes called the "Schwarzian" limit because in this case the boundary dynamics is governed by [2-4]

$$
S_{\mathrm{Sch}}[t]=-\int d u\left\{e^{t(u)}, u\right\}, \quad\{f, u\}=\frac{f^{\prime \prime \prime}}{f^{\prime}}-\frac{3}{2} \frac{f^{\prime \prime 2}}{f^{\prime 2}}, \quad d \tau_{p}=2 \phi_{b} d u
$$

where $u$ is a rescaled version of proper time $\tau_{p}$, and $t$ can be viewed as the Rindler time $t$ in (2.6a) near the boundary. We can view the curve $t(u)$ as parametrizing the position of the boundary. The action (2.13) captures a gravitational degree of freedom that we can view as living on the boundary. ${ }^{5}$

We will consider spacetimes describing a two sided eternal black hole, so that we have two boundaries and two variables $t_{r}, t_{l}$, each with the action (2.13). The dynamics of the full system (2.12) reduces to the dynamics of three decoupled systems connected only by an overall $\mathrm{SL}(2)_{g}$ constraint

$$
S=S_{\mathrm{Sch}}\left[t_{r}\right]+S_{\mathrm{Sch}}\left[t_{l}\right]+S_{m}\left[g_{\mu \nu}, \chi\right]
$$

These three decoupled systems are the following. First we have the matter which lives in exactly $A d S_{2}$ space and has $\operatorname{SL}(2)_{g}$ charges $Q_{m}^{a}$. Then we have the right and left boundaries. In this limit, they are not directly coupled to each other or to the matter. However, in $\mathrm{NAdS}_{2}$ gravity, an overall $\mathrm{SL}(2)_{g}$ transformation is a redundancy of our description. Hence the physical Hilbert space is [2-4]

$$
\mathcal{H}_{\text {Physical }}=\left(\mathcal{H}_{l} \times \mathcal{H}_{\text {matter }} \times \mathcal{H}_{r}\right) / \operatorname{SL}(2)_{g}, \quad Q_{l}^{a}+Q_{m}^{a}+Q_{r}^{a}=0
$$

where the charges $Q^{a}$ are the $\mathrm{SL}(2)_{g}$ charges of each of the systems. Physically, this says that only the relative positions of the matter and the boundaries matter. As pointed out in [11], we can view it as "Mach's" principle, where the boundaries are the "distant stars". These are part of the usual constraints of general relativity.

One can find explicit expressions for the $\mathrm{SL}(2)_{g}$ charges of the right and left boundaries by using the Noether procedure on (2.13) [3]

$$
\begin{aligned}
Q_{r}^{-1} & =\frac{t_{r}{ }^{\prime \prime \prime}}{t_{r}{ }^{2}}-\frac{t_{r}{ }^{\prime 2}}{t_{r}{ }^{3}}-t_{r}{ }^{\prime} \\
Q_{r}^{+} & =e^{t_{r}}\left[\frac{t_{r}{ }^{\prime \prime \prime}}{t_{r}{ }^{2}}-\frac{t_{r}{ }^{\prime 2}}{t_{r}{ }^{3}}-\frac{t_{r}{ }^{\prime \prime}}{t_{r}{ }^{\prime}}\right] \\
Q_{r}^{-} & =e^{-t_{r}}\left[-\frac{t_{r}^{\prime \prime \prime}}{t_{r}{ }^{2}}+\frac{t_{r}{ }^{\prime 2}}{t_{r}{ }^{3}}-\frac{t_{r}{ }^{\prime \prime}}{t_{r}{ }^{\prime}}\right] .
\end{aligned}
$$

\footnotetext{
${ }^{5}$ This should not be confused with a possible holographically dual boundary quantum mechanical theory, which would describe the full system.
} 
where $Q^{ \pm}=Q^{0} \pm Q^{1}$. The left side charges may also be obtained by analytic continuation $Q_{l} \rightarrow-Q_{r}$ with $t_{l}=-t_{r}+i \pi, u_{l} \rightarrow-u_{r}+i$ (constant). We are defining $u_{l}$ and $t_{l}$ so that they go forwards in time in the thermofield double interpretation.

$$
\begin{aligned}
& Q_{l}^{-1}=-\frac{t_{l}^{\prime \prime \prime}}{t_{l}{ }^{2}}+\frac{t_{l}{ }^{\prime 2}}{t_{l}{ }^{\prime 3}}+t_{l}^{\prime} \\
& Q_{l}^{+}=e^{-t_{l}}\left[\frac{t_{l}{ }^{\prime \prime \prime}}{t_{l}{ }^{\prime 2}}-\frac{t_{l}^{\prime 2}}{t_{l}{ }^{\prime 3}}+\frac{t_{l}{ }^{\prime \prime}}{t_{l}^{\prime}}\right] \\
& Q_{l}^{-}=e^{t_{l}}\left[-\frac{t_{l}^{\prime \prime \prime}}{t_{l}{ }^{\prime 2}}+\frac{t_{l}{ }^{\prime 2}}{t_{l}{ }^{\prime 3}}+\frac{t_{l}^{\prime \prime}}{t_{l}^{\prime}}\right]
\end{aligned}
$$

One can check that $\frac{1}{2} Q_{r} \cdot Q_{r}=\left\{e^{-t_{r}}, u\right\}$.

For our subsequent discussion it is convenient to write a nicer expression for the boundary position so that its $\mathrm{SL}(2)$ transformations properties are more manifest. The dynamics of the boundary is closely related to the dynamics of a charged massive particle, or a particle with spin, in the limit that the mass and the charge (or spin) becomes both very large, while keeping the total $\operatorname{SL}(2)$ charges $Q^{a}$ finite $[10,11]$. We have $(2.9)$ with $\mathfrak{q}=2 \phi_{b}$. In this case, the coordinates $Y^{a}$ become very large because we approach the boundary. So it is convenient to define rescaled coordinates $X^{a}$ via

$$
X_{r}^{a}=\frac{Y_{r}^{a}}{Y_{r} \cdot Q_{r}}=\frac{Y_{r}^{a}}{\mathfrak{q}}, \quad X_{r} \cdot Q_{r}=1, \quad X_{r}^{2} \rightarrow 0
$$

So, from the point of view of $(2.9)$ we have $r^{2}=0, \mathfrak{q}_{x}=1$ for the variable $X_{r}^{a}$. For $X_{l}$ we get $\mathfrak{q}_{x}=-1$. We can also rescale proper time by the same factor so that now we obey $\dot{X}_{r} \cdot \dot{X}_{r}=-1$. In terms of our previous variables these can be written as

$$
\begin{aligned}
& X_{r}=\left(X^{-1}, X^{+}, X^{-}\right)=\left(\frac{1}{t_{r}}, \frac{e^{t_{r}}}{t_{r}{ }^{\prime}},-\frac{e^{-t_{r}}}{t_{r}{ }^{\prime}}\right), \quad X^{ \pm} \equiv X^{0} \pm X^{1} \\
& X_{l}=\left(\frac{1}{t_{l}{ }^{\prime}},-\frac{e^{-t_{l}}}{t_{l^{\prime}}}, \frac{e^{t_{l}}}{t_{l^{\prime}}}\right) .
\end{aligned}
$$

We can check that $-X_{l} \cdot Q_{l}=X_{r} \cdot Q_{r}=1$. In appendix B, we verify that in the canonically quantized Schwarzian theory, the above operators satisfy the Poincare algebra (2.9) with the appropriate Casimirs $r^{2}=0, \mathfrak{q}_{x}= \pm 1$. (In appendix D we give an alternative description in terms of spinors.) In addition, the Hamiltonian corresponding to the Schwarzian action $(2.13)$ is

$$
2 H_{r}=-Q_{r}^{2}=\ddot{X}_{r}^{2} .
$$

Using (2.9) this gives us the quantum mechanical relation

$$
\dot{X}_{r}^{a}=i\left[H_{r}, X_{r}^{a}\right]=-\frac{i}{2}\left[Q_{r}^{2}, X_{r}^{a}\right]=\frac{1}{2} \epsilon_{b c}^{a}\left(X_{r}^{b} Q_{r}^{c}+Q_{r}^{b} X_{r}^{c}\right) .
$$

Taking a second derivative we get the operator equations

$$
\begin{array}{rlrl}
Q_{r}^{a} & =\ddot{X}_{r}^{a}-H_{r} X_{r}^{a}-X_{r}^{a} H_{r}, & X_{r} \cdot Q_{r}=1, & \dot{X}_{r}^{2}=-1, \\
Q_{l}^{a}=-\ddot{X}_{l}^{a}+H_{l} X_{l}^{a}+X_{l}^{a} H_{l}, & X_{l} \cdot Q_{l}=-1, & \dot{X}_{l}^{2}=-1,
\end{array}
$$


where the difference in signs is due to the difference in sign of $\mathfrak{q}$ for the left boundary. We may also use the algebra to compute commutators between $X, \dot{X}, \ddot{X}$. For example,

$$
\left[\dot{X}_{r}^{a}, X_{r}^{b}\right]=-i X_{r}^{a} X_{r}^{b}
$$

Using these coordinates it is also possible to write the correlation functions of operators dual to matter fields in the bulk. If we have a massive field in the bulk giving rise to an operator of dimension $\Delta$, then its left right correlator is given by

$$
\left\langle O\left(u_{l}\right) O\left(u_{r}\right)\right\rangle \propto \frac{1}{\left(-2 X_{l}\left(u_{l}\right) \cdot X_{r}\left(u_{r}\right)\right)^{\Delta}}=\left(\frac{t_{l}^{\prime}\left(u_{l}\right) t_{r}^{\prime}\left(u_{r}\right)}{4 \cosh ^{2}\left(\frac{t_{l}\left(u_{l}\right)+t_{r}\left(u_{r}\right)}{2}\right)}\right)^{\Delta}
$$

where we used (2.19). We get a similar formula for correlators on the same side.

\section{$2.3 \quad$ Review of SYK}

The SYK model contains $N$ Majorana fermions with random interactions affecting $q$ fermions at a time, $q=4,6, \cdots[5-8]$. In the large $N$ limit, one can write down an effective action in terms of a bilocal field $G\left(u_{1}, u_{2}\right)$, which becomes equal to the average two point function once we impose the equations of motion. At low energies this action becomes nearly reparameterization-invariant, except for a low action reparametrization mode (or soft mode), which has a Schwarzian action with an overall coefficient scaling as $N / J$, with $J$ an energy scale setting the strength of the interactions of the original model.

In more detail, we start with a scaling solution

$$
G_{0}\left(t_{1}, t_{2}\right) \propto\left|t_{1}-t_{2}\right|^{-2 \Delta}
$$

The soft mode corresponds to functions $G$ obtained by a reparametrization of (2.26), $G=\left[f^{\prime}\left(u_{1}\right) f^{\prime}\left(u_{2}\right)\right]^{\Delta} G\left(f\left(u_{1}\right), f\left(u_{2}\right)\right)$. We can also generate new configurations by having fluctuations $\delta_{\perp} G\left(t_{1}, t_{2}\right)$ which lie in the directions orthogonal to the soft mode. For now the coordinates $t_{1}$ and $t_{2}$ are some coordinates that appear in the solution of the low energy equations of the SYK model and are defined by the form of the unperturbed solution (2.26). We introduce the soft mode by writing the full physical $G$ as [8]

$$
G\left(u_{1}, u_{2}\right)=\left[f^{\prime}\left(u_{1}\right) f^{\prime}\left(u_{2}\right)\right]^{\Delta} G_{0}\left(f\left(u_{1}\right), f\left(u_{2}\right)\right)+\left[f^{\prime}\left(u_{1}\right) f^{\prime}\left(u_{2}\right)\right]^{\Delta} \delta_{\perp} G\left(f\left(u_{1}\right), f\left(u_{2}\right)\right)
$$

This can be viewed as parametrization the full space of functions $G$. Namely, we think of the integration variables as $f(u)$ and $\delta G_{\perp}\left(t_{1}, t_{2}\right)$. Inserting this expression into the SYK action, and taking a low energy limit, we find that the full (Euclidean) action becomes

$$
S=S\left[G_{0}\left(t_{1}, t_{2}\right)+\delta_{\perp} G\left(t_{1}, t_{2}\right)\right]-\frac{N \alpha_{S}}{\mathcal{J}} \int d u\{f, u\}
$$

where we used the approximate reparametrization invariance of the low energy action. This means that the first term in (2.28) is independent of $f$. All the dependence on $f$ is in the second term of (2.28) and it comes from a small violation of the reparametrization 
symmetry [8]. To evaluate the path integral, one should sum over different $f$ and $\delta_{\perp} G$. An important point is that in this parametrization, we have an $\mathrm{SL}(2)_{g}$ symmetry

$$
f \rightarrow \frac{a f+b}{c f+d}, \quad \delta_{\perp} G\left(t_{1}, t_{2}\right) \rightarrow \frac{1}{\left[\left(a-c t_{1}\right)\left(a-c t_{2}\right)\right]^{2 \Delta}} \delta_{\perp} G\left(\frac{d t_{1}-b}{-c t_{1}+a}, \frac{d t_{2}-b}{-c t_{2}+a}\right)
$$

The arguments of $\delta_{\perp} G$ are transforming in the inverse way than $f$ so that the second term in (2.27) remains invariant. The first term in (2.27) also remains invariant under this transformation. Therefore (2.29) is a redundancy in our parametrization of the space of $G\left(u_{1}, u_{2}\right)(2.27)$ and we should demand that everything is invariant. Note that when we write the action as the sum of two terms such as in (2.28) (or three terms if we wrote the Lorentzian action for the thermofield double), then the $\mathrm{SL}(2)_{g}$ symmetry will act in a non-trivial way on the variables of each term. In particular, the $\mathrm{SL}(2)_{g}$ action transforms $\delta_{\perp} G\left(t_{1}, t_{2}\right)$, as in (2.29). So, even though the two terms of the action (2.28) are decoupled, they become connected by the total $\mathrm{SL}(2)_{g}$ constraint.

The conclusion is that in the SYK model we have a structure which is similar to the one we had in nearly $\mathrm{AdS}_{2}$ gravity. We have three separate systems connected by an overall gauge constraint. The Schwarzian parts are identical to what we had in gravity. But the analog of the matter action $S_{m}\left[g_{\mu \nu}, \chi\right]$ is the first term in (2.28). It is independent of the Schwarzian variables, but its variables transform nontrivially under $\mathrm{SL}(2)_{g}$.

\section{Exact generators}

\subsection{Construction of gauge invariant $\mathrm{SL}(2)$ generators}

In $\mathrm{NAdS}_{2}$ gravity, bulk matter "feels" as if it was moving in empty $A d S_{2}$. This suggests that we could define $\mathrm{SL}(2)$ generators that move the matter. Naively these would be $Q_{m}^{a}$. However, these are not physical because they are not invariant under the SL(2) gauge symmetry. Said slightly differently, once we go from quantum field theory on a fixed background to quantum gravity, we must gravitationally dress all observables. Since the metric of $\mathrm{NAdS}_{2}$ is essentially rigid, the dressing should involve the boundary degrees of freedom.

For example, given two boundary positions $X_{l}^{a}$ and $X_{r}^{a}$ we can define the vector $W^{a}=$ $\epsilon^{a b c} X_{l b} X_{r c}$ and the generator

$$
G^{0}=\tilde{P}=\frac{\epsilon_{a b c} Q_{m}^{a} X_{l}^{b} X_{r}^{b}}{X_{l} \cdot X_{r}}
$$

where we introduced two different notations for the generator. This generator leaves the boundary points $X_{l}$ and $X_{r}$ invariant. It is a translation in the bulk along the geodesic that joins these two boundary points, see figure 2 . In addition, we have normalized it so that it generates translations by a "unit" proper distance in the bulk. In the case that $X_{l}$ and $X_{r}$ correspond to the points with $T=0$ and $\sigma=0, \pi$ in (2.6a) get we the generator $P$ in (2.5). For general positions for $X_{l}$ and $X_{r}$ we get a linear combination of the generators in (2.5). A nice feature of (3.1) is that it is invariant under the $\mathrm{SL}(2)$ gauge transformations. Another nice feature of (3.1) is the fact that it acts within the so called "Wheeler-de-Witt" patch, 

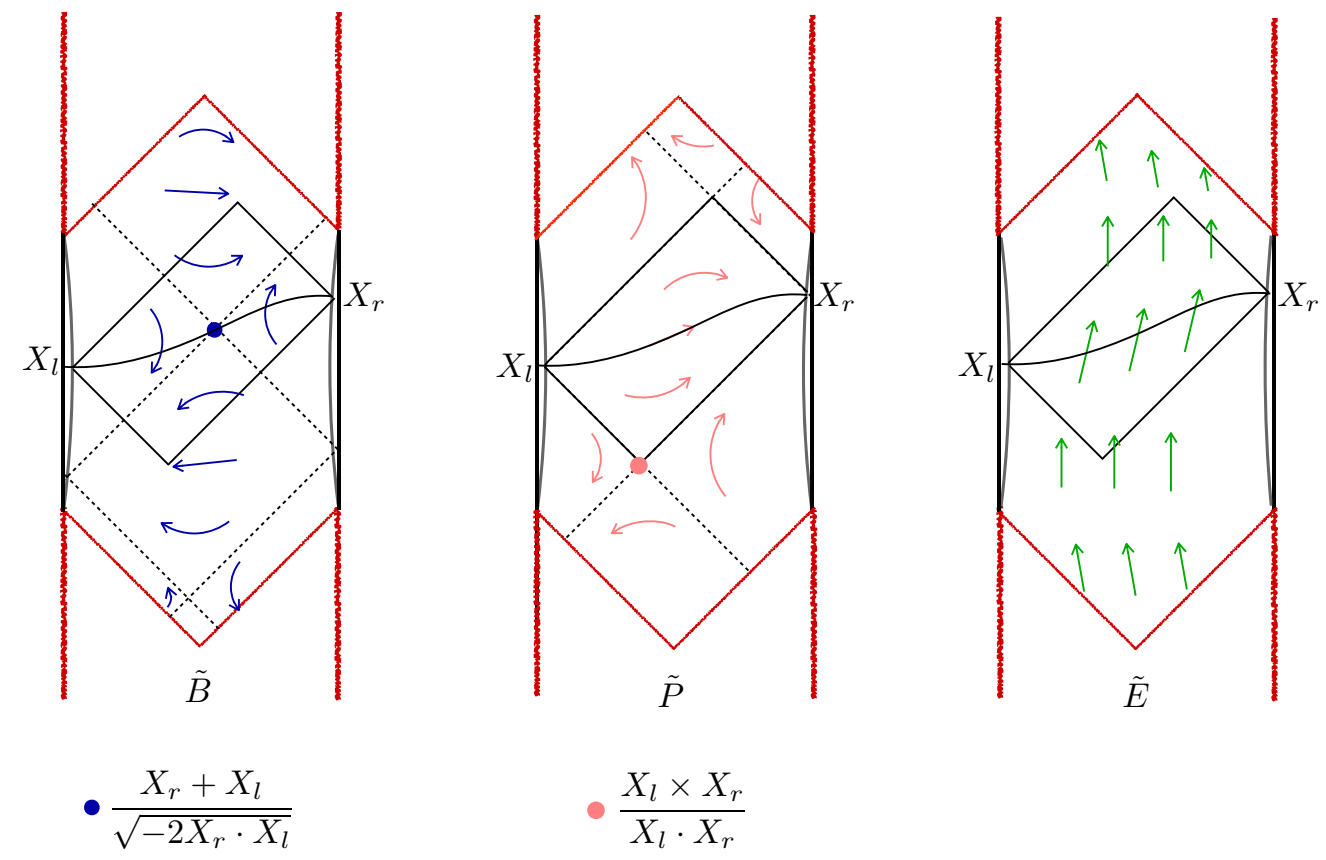

Figure 2. Geometrical action of the gauge invariant charges $\tilde{P}, \tilde{B}, \tilde{E}$. The points which are fixed by the symmetry generators are also indicated.

which is the set of points that are spacelike separated from both boundary points, $X_{l}$ and $X_{r}$, see figure 2 .

We can now wonder whether we can define two other generators in a similar way. Natural candidates are

$$
G^{1}+G^{-1}=\tilde{E}+\tilde{B}=-2 \frac{Q_{m}^{a} X_{l a}}{\sqrt{-2 X_{l} \cdot X_{r}}}, \quad G^{1}-G^{-1}=\tilde{E}-\tilde{B}=2 \frac{Q_{m}^{a} X_{r a}}{\sqrt{-2 X_{l} \cdot X_{r}}}
$$

These are generators which leave one of the points fixed, ( $X_{l}$ for the first, and $X_{r}$ for the second). They do not act within the Wheeler de Witt patch, and can map points inside to points outside, see figure 2 . These generators have been defined so that they obey the same algebra as the generators in (2.5), but are defined relative to the two boundary positions. Notice that they involve a matter operator, $Q_{m}^{a}$ and operators of the boundary systems $X_{l, r}^{a}$. Since they are gauge invariant, they map physical states to other physical states.

We can think of the generators $\tilde{B}$ as defined by the following procedure. Imagine that have two points $X_{l}$ and $X_{r}$ that are very far away, but not yet at the boundary. Then we join them by a geodesic and determine their midpoint. Then $\tilde{B}$ is the boost around this midpoint. Then the third generator, $\tilde{E}$, results from commuting the previous ones and gives a generator that locally looks like a time translations around the midpoint, see figure 2. These are time translations locally orthogonal to the geodesic joining $X_{l}$ and $X_{r}$.

Acting on a state with given boundary coordinates $X_{l}$ and $X_{r}$, this state moves the matter around leaving the boundary points fixed. The resulting time evolution of $X_{l}$ and $X_{r}$ can be changed by the action of these generators, but not their instantaneous positions. 
We have found the action of a physical SL(2) symmetry on the physical Hilbert space. In particular this means that the physical Hilbert space is infinite dimensional due to the matter degrees of freedom, and their descendants.

In this discussion, we have neglected the possibility of topology changes, such as the ones in [26], since we assumed that the topology is essentially a strip. Therefore we are assuming that $\phi_{0}$ in (2.12) is very large so that topology changes are highly suppressed. It would be interesting to understand how other topologies change the picture; presumably it should be related to cutting off the algebra to a finite dimensional Hilbert space.

An alternative way to describe this same construction is to say that we have defined three vectors $e_{a}^{A}$, where $A$ is an index running over the three vectors, and then we defined three gauge invariant generators

$$
G^{A}=e_{a}^{A} Q_{m}^{a} .
$$

The three vectors were the ones in (3.1) (3.2), i.e.,

$$
\begin{aligned}
e_{a}^{0} & =\frac{\epsilon_{a b c} X_{l}^{b} X_{r}^{c}}{X_{l} \cdot X_{r}}, \quad e_{a}^{-1}=-\frac{1}{\sqrt{-2 X_{l} \cdot X_{r}}}\left(X_{r a}+X_{l a}\right), \\
e_{a}^{1} & =\frac{1}{\sqrt{-2 X_{l} \cdot X_{r}}}\left(X_{r a}-X_{l a}\right) .
\end{aligned}
$$

The $G^{A}$ also obey the SL(2) algebra, $\left[G^{A}, G^{B}\right]=i \epsilon^{A B C} G_{C}$, due to the properties of $e_{a}^{A}$ and the commutation relations of $Q_{m}^{a}$. We can also write the matter Casimir

$$
C \equiv G^{A} G^{B} \eta_{A B}=Q_{m}^{a} Q_{m}^{b} \eta_{a b}=\tilde{E}^{2}-\tilde{B}^{2}-\tilde{P}^{2}=E_{m}^{2}-B_{m}^{2}-P_{m}^{2}
$$

which is $\mathrm{SL}(2)$ gauge invariant and commutes with the Hamiltonian.

As a side comment, we may preserve the algebra by rescaling $E, B, P$ by a factor depending on $X_{l} . X_{r}$ if we also rescale $\eta$ by a compensating factor.

\subsubsection{Writing the charges purely in terms of boundary quantities}

We can use the fact that $Q_{m}^{a}=-\left(Q_{r}^{a}+Q_{l}^{a}\right),(2.15)$, together with (2.21), to write

$$
\begin{aligned}
G^{0}=\tilde{P} & =-\frac{\epsilon_{a b c} X_{l}^{b} X_{r}^{c}\left(Q_{r}^{a}+Q_{l}^{a}\right)}{X_{l} \cdot X_{r}}=\frac{\dot{X}_{l} \cdot X_{r}-X_{l} \dot{X}_{r}}{X_{l} \cdot X_{r}} \\
\tilde{P} & =\left(\partial_{u_{l}}-\partial_{u_{r}}\right) \log \left[-2 X_{l} \cdot X_{r}\right]=\left(\partial_{u_{l}}-\partial_{u_{r}}\right) \ell \\
\tilde{P} & =\frac{\alpha_{S} N}{\mathcal{J}}\left(\partial_{u_{l}}-\partial_{u_{r}}\right) \ell=\frac{r_{e}^{3}}{G_{N}}\left(\partial_{u_{l}}-\partial_{u_{r}}\right) \ell
\end{aligned}
$$

where we noted that $\log \left[-2 X_{l} . X_{r}\right]$ is the regularized distance between the two boundaries, in units of the radius of $\mathrm{AdS}_{2}$. By "regularized" we mean that we have subtracted an infinite additive constant to the actual proper distance. ${ }^{6}$ In (3.7) we have restored the constants that we had set to one for the SYK case or the $4 \mathrm{~d}$ near extremal charged black hole.

Notice that, due to (2.24), the numerator commutes with the denominator, even though each term in the numerator does not commute with the denominator. In this formula, (3.6),

\footnotetext{
${ }^{6}$ This infinite constant is independent of time and independent of the $X_{l}$ or $X_{r}$ variables.
} 
we see that the total momentum is expressed purely in terms of boundary quantities, or gravitational quantities. ${ }^{7}$ In addition, this generator can be interpreted as the matter momentum in a frame set by the boundary positions.

Notice that (3.6) is a rather pleasing expression because it can be interpreted as saying that the momentum of matter is minus the momentum of the left plus right boundaries. Namely, if we choose a coordinate $x$ along the geodesic connecting the two boundaries, then the distance is $\ell=x_{r}-x_{l}$ and the momentum is

$$
\tilde{P}=\left(\partial_{u_{l}}-\partial_{u_{r}}\right)\left[x_{r}\left(u_{r}\right)-x_{l}\left(u_{l}\right)\right]=-\left(\dot{x}_{r}+\dot{x}_{l}\right) .
$$

This is saying that the matter momentum is minus the sum of the momenta of the boundary particles. We get the naive expression for the momentum of the boundary particles because the term involving $\mathfrak{q}$ in $(2.8)$ drops out when we contract $\vec{Y}_{l} \times \vec{Y}_{r}$ with $\vec{Q}$. So we get the same result as for an ordinary massive particle.

Note that in writing (3.6) we assumed a particular form for the Hamiltonian that generates the $u$ dependence. In particular, we have assumed that we have a decoupled evolution, by $H_{l}$ and $H_{r}$ in (2.20). In contrast, the expressions (3.1) (3.2) did not use the form of the Hamiltonian and are valid more generally (for example we could have a small coupling between the left and right sides). We can obtain expressions that are more generally valid by writing $Q_{l}^{a}$ and $Q_{r}^{a}$ in terms of the boundary positions and their conjugate momenta, see appendix B and (B.7).

We can also consider the expressions for the other generators. Again, we start from (3.2) and we express the matter charges in terms of the left and right charges, and use (2.22) (2.23) to obtain (ignoring operator ordering issues)

$$
\begin{aligned}
G^{1} & =\tilde{E}=\left(-2 X_{l} \cdot X_{r}\right)^{1 / 2}\left(H_{l}+H_{r}+\frac{1}{X_{l} \cdot X_{r}}-\frac{\ddot{X}_{l} \cdot X_{r}+X_{l} \cdot \ddot{X}_{r}}{2 X_{l} \cdot X_{r}}\right), \\
G^{-1} & =\tilde{B}=-\left(-2 X_{l} \cdot X_{r}\right)^{1 / 2}\left(H_{l}-H_{r}+\frac{X_{l} \cdot \ddot{X}_{r}-\ddot{X}_{l} \cdot X_{r}}{2 X_{l} \cdot X_{r}}\right) .
\end{aligned}
$$

We can also express the Casimir (3.5) in terms of purely boundary quantities. Of course, these expressions depend on both boundaries.

These observables can be expressed in terms of energies and distances between left and right sides. We will discuss how to measure distances in section 7.1.

\subsection{Defining "conserved" charges}

The generators we have defined above act on the physical Hilbert space but they do not commute with the Hamiltonians of the system, $H_{l}$ or $H_{r}$. Therefore we cannot call them conserved quantities. (Of course, the Casimir (3.5) is indeed conserved.) However, one feature of the gauge-non-invariant matter charges is that they are conserved $\left[H_{l, r}, Q_{m}^{a}\right]=0$ in the unphysical Hilbert space.

\footnotetext{
${ }^{7}$ Note that we are talking about the boundary gravitational degrees of freedom, and not the holographically dual boundary quantum mechanical theory.
} 
The charges we defined depend on the left and right times through the boundary positions $X_{l}\left(u_{l}\right)$ and $X_{r}\left(u_{r}\right)$. Then the charges in (3.1) (3.2) depend on the two times $G^{A}\left(u_{l}, u_{r}\right)$. However, the dynamics of the left and right boundaries is solvable as a quantum mechanical theory. This means that the change in the charges follows a reasonably predictive pattern. In particular, we would obtain time independent expressions for the generators by solving the boundary dynamics so that we can work with $X_{l}(0)$ and $X_{r}(0)$. Therefore we can simply say that the "conserved" charges are simply $G^{A}\left(0_{l}, 0_{r}\right)$ where we have set both times to zero. Now, this looks like we are cheating since we can always define a conserved quantity by undoing the time evolution. However, in this case, the statement has non-trivial content because we only have to undo the evolution of the boundary mode, the Schwarzian degree of freedom. In particular, we are not undoing the evolution of matter, which could be a complicated self interacting theory. In addition, in the classical limit, we can undo the classical evolution of the boundary theory in a simple way. In principle, we can also express $G^{a}(0,0)$ in terms of the correlators at zero as in (3.6) (3.9).

Formally we can write down

$$
G^{A}(0,0)=e^{-i\left(H_{l} u_{l}+H_{r} u_{r}\right)} G^{A}\left(u_{l}, u_{r}\right) e^{i\left(H_{l} u_{l}+H_{r} u_{r}\right)}=\Lambda_{B}^{A} G^{B}\left(u_{l}, u_{r}\right)
$$

with

$$
\Lambda_{B}^{A}=e_{a}^{A}(0,0)\left(e^{-1}\left(u_{l}, u_{r}\right)\right)_{B}^{a}=e_{a}^{A}(0,0) \eta^{a b} e_{b}^{C}\left(u_{l}, u_{r}\right) \eta_{C B} .
$$

This expression for $\Lambda$ involves the quantum operators $X_{l, r}$ evaluated at zero and also evaluated at $u_{l}, u_{r}$, so it is a rather complex expression in the quantum Schwarzian theory. Note that the operator $X_{r}(0)$ can be expressed explicitly in terms of operators at time $u_{l}, u_{r}$ by using the propagators for the Schwarzian theory [10, 11]. Unfortunately, the operators are not diagonal in the $X_{r}\left(u_{r}\right)$ basis, so it is hard to express them in terms of correlators at time $u_{r}$, and we will not attempt to do it here.

Let us mention that even the standard expressions for the matter charges (2.11) involve some explicit time dependent expressions, since (some of) the Killing vectors depend explicitly on time. In (2.11), this dependence is very simple. In our problem the time dependence is a bit more complicated, but in principle solvable.

One case where the dynamics can be solved simply is the classical limit, as we will see in (4.8).

\section{Approximate expressions for the generators}

\subsection{The generators in the semiclassical limit}

It is instructive to consider the above construction in the semiclassical limit. So we start with a two sided black hole solution with $\beta \ll 1$,

$$
t_{r}=\tilde{u}, \quad t_{l}=\tilde{u}, \quad \tilde{u} \equiv \mathfrak{s} u, \quad \mathfrak{s} \equiv \frac{2 \pi}{\beta}=\frac{2 \pi \alpha_{S} N}{\beta \mathcal{J}}=\frac{2 \pi r_{e}^{3}}{\beta G_{N}}
$$

where we have defined the coefficient of the Schwarzian action $\mathfrak{s}$, which has the interpretation of the $\mathrm{SL}(2)$ spin of the state in the Schwarzian theory $\left(j=\frac{1}{2}+i \mathfrak{s}\right)$. It is also related 
to the near extremal entropy, $S-S_{0}=2 \pi \mathfrak{s}$. We have also restored the constants we had set to one for the case of SYK or $4 \mathrm{~d}$ near extremal charged black holes.

It is common in these discussions to keep two parameters $N$ and $\mathcal{J}$ as independent, and the fact that we have removed them completely might confuse some readers. Indeed they are independent parameters in a model such as $S Y K$. However, all of our discussion is centering on the low energy regime and the coupling of the Schwarzian theory to the conformal sector. These parameters appear only in the overall coefficient of the Schwarzian action. We have rescaled the units of time $u$, so as to set this constant to one, see (2.13). This highlights the fact that there is only an overall lengthscale appearing the problem, and we have chosen units where this lengthscale is set to one. This is the timescale at which the Schwarzian theory becomes strongly coupled. In these units the limit $\beta \ll 1$ corresponds to the semiclassical limit of the Schwarzian theory. We can restore the full dependence on $N / \mathcal{J}$ by restoring such constants by using dimensional analysis. For example, thinking of $\mathfrak{s}$ as the entropy we restore them as in (4.1). If we had $\beta$ appearing in a dimensionless quantity, such as $e^{2 \pi u / \beta}$, then no further change is needed, since the rescaling of $u$ and $\beta$ cancel out. The semiclassical limit corresponds to $\mathfrak{s} \gg 1$.

Inserting (4.1) into the right-left charges (2.16) (2.17) we find that $Q_{r}^{a}+Q_{l}^{a}=0$ as expected. The only non-zero components of these charges are $Q_{l}^{-1}=-Q_{r}^{-1}=\mathfrak{s}$. We now add a relatively small amount of bulk matter $Q_{m}$. By small we mean that the changes in the boundary trajectories are small,

$$
t_{r}=\tilde{u}+\epsilon_{r}(\tilde{u}), \quad t_{l}=\tilde{u}+\epsilon_{l}(\tilde{u})
$$

with $\epsilon_{r, l} \ll 1$. In this case we can expand the charges $Q_{r}^{a}, Q_{l}^{a}$ in $\epsilon$. In fact, it is convenient to expand the sum of the charges because this sum is then equated to $Q_{m}^{a}=-\left(Q_{l}^{a}+Q_{r}^{a}\right)$.

This gives

$$
\begin{aligned}
Q_{m}^{-1} & \simeq \mathfrak{s}\left[\epsilon_{r}^{\prime}-\epsilon_{r}^{\prime \prime \prime}-\epsilon_{l}^{\prime}+\epsilon_{l}^{\prime \prime \prime}\right], \quad \text { where } \quad{ }^{\prime} \equiv \partial_{\tilde{u}} \\
Q_{m}^{0}+Q_{m}^{1}=Q_{m}^{+} & \simeq \mathfrak{s}\left[e^{\tilde{u}_{r}}\left(\epsilon_{r}^{\prime \prime}-\epsilon_{r}^{\prime \prime \prime}\right)+e^{-\tilde{u}_{l}}\left(-\epsilon_{l}^{\prime \prime}-\epsilon_{l}^{\prime \prime \prime}\right)\right]=\mathfrak{s}\left[\epsilon_{r}^{\prime \prime}(0)-\epsilon_{r}^{\prime \prime \prime}(0)-\epsilon_{l}^{\prime \prime}(0)-\epsilon_{l}^{\prime \prime \prime}(0)\right] \\
Q_{m}^{0}-Q_{m}^{1}=Q_{m}^{-} & \simeq \mathfrak{s}\left[e^{-\tilde{u}_{r}}\left(\epsilon_{r}^{\prime \prime}+\epsilon_{r}^{\prime \prime \prime}\right)+e^{\tilde{u}_{l}}\left(-\epsilon_{l}^{\prime \prime}+\epsilon_{l}^{\prime \prime \prime}\right)\right]=\mathfrak{s}\left[\epsilon_{r}^{\prime \prime}(0)+\epsilon_{r}^{\prime \prime \prime}(0)-\epsilon_{l}^{\prime \prime}(0)+\epsilon_{l}^{\prime \prime \prime}(0)\right]
\end{aligned}
$$

where the primes on $\epsilon_{l}\left(\tilde{u}_{l}\right)$ and $\epsilon_{r}\left(\tilde{u}_{r}\right)$ are derivatives with respect to $\tilde{u}_{l}, \tilde{u}_{r}$ respectively. These expressions are naively $u$ dependent, but the equations of motion for $\epsilon$ make sure that they are $u$ independent, and we have given the expressions for zero times. Namely, from the conservation of energy,

$$
H_{r}=\frac{\mathfrak{s}^{2}}{2}+\mathfrak{s}^{2}\left(\epsilon_{r}^{\prime}-\epsilon_{r}^{\prime \prime \prime}\right)+\cdots, \quad H_{l}=\frac{\mathfrak{s}^{2}}{2}+\mathfrak{s}^{2}\left(\epsilon_{l}^{\prime}-\epsilon_{l}^{\prime \prime \prime}\right)+\cdots
$$

we get

$$
\epsilon^{\prime \prime \prime \prime}-\epsilon^{\prime \prime}=0, \quad \epsilon=\epsilon_{r, l}\left(\tilde{u}_{l, r}\right)
$$

which ensures that the right hand sides of (4.3) are all independent of time (as are the left hand sides).

Inserting (4.1) into (2.19) and then computing the generators $G^{A}$ to zeroth order in $\epsilon$ we find

$$
G^{A}(0,0) \simeq Q_{m}^{a}, \quad A=a
$$


This equation is valid in the gauge we used to write the background solution (4.1). In general we can also compute the generators $G^{A}$ at more general times. Using the classical evolution to evolve the vectors $e_{a}^{A}$ we can express them as a linear combination of the ones in (4.6), see (3.11),

$$
G^{A}(0,0) \simeq \Lambda_{B}^{A} G^{B}\left(u_{l}, u_{r}\right)
$$

with

$$
\begin{aligned}
\Lambda & =\left(\begin{array}{ccc}
1 & 0 & 0 \\
0 & \cosh \gamma & \sinh \gamma \\
0 & \sinh \gamma & \cosh \gamma
\end{array}\right) \cdot\left(\begin{array}{ccc}
\cos \alpha & -\sin \alpha & 0 \\
\sin \alpha & \cos \alpha & 0 \\
0 & 0 & 1
\end{array}\right), \\
\text { where } \quad \sin \alpha & =\tanh \frac{\tilde{u}_{l}+\tilde{u}_{r}}{2}, \quad \gamma=\frac{\tilde{u}_{l}-\tilde{u}_{r}}{2} .
\end{aligned}
$$

This is reflecting the fact that when we pick arbitrary left and right times, in the classical limit, the generators $G^{A}$ have been rotated relative to the ones at zero times, see figure 2 . In this case, the time dependence of the generators is simple and can be extracted to define the time independent generators $G^{A}(0,0)$. This is the classical version of the formula (3.11).

It is also interesting to note that we can also obtain (4.3) by evaluating $G^{A}\left(u_{l}, u_{r}\right)$ using correlators, as in (3.6) and (3.9). In detail, what we have in mind is the following. Let us consider (3.6), for example. We express $X_{l} . X_{r}$ in terms of the boundary times as in (2.25). We then expand the times as in (4.2), to obtain

$$
\tilde{P}\left(u_{l}, u_{r}\right)=\left(\partial_{u_{l}}-\partial_{u_{r}}\right) \log \left[-2 X_{l} \cdot X_{r}\right]=\mathfrak{s}\left[\epsilon_{r}^{\prime \prime}-\epsilon_{l}^{\prime \prime}+\frac{1}{2}\left(\epsilon_{l}^{\prime}-\epsilon_{r}^{\prime}\right) \tanh \frac{\tilde{u}_{l}+\tilde{u}_{r}}{2}\right] .
$$

In a similar way we get can get the other generators. Then applying the inverse of the matrix in (4.8) we get the generators $G^{A}(0,0)$ which are the ones in (4.6), with the expression (4.3).

\subsection{The semiclassical limit and SL(2) symmetries of the physical boundary time}

In this semiclassical limit, we can think of the $G^{A}(0,0)$ as generating a symmetry that acts as ordinary reparametrizations of $\tilde{u}$, generated by the infinitesimal transformations

$$
\mathrm{SL}(2)_{u}: \quad \tilde{u} \rightarrow \tilde{u}+\alpha_{-1}+\alpha_{+} e^{\tilde{u}}+\alpha_{-} e^{-\tilde{u}}, \quad \tilde{u}=\mathfrak{s} u, \quad \mathfrak{s}=\frac{2 \pi}{\beta}
$$

In order to make this manifest we will analyze how the charges $G^{A}(0,0)$ act on states created by the insertion of operators in Euclidean time. In fact, we will discuss a state/operator map for the nearly- $C F T_{1}$ that is dual to this gravity theory.

More precisely, we view the state at $u_{l}=u_{r}=0$ as created by Euclidean time evolution over a time $\Delta u_{e}=\beta / 2$ (or $\delta \tilde{u}_{e}=\pi$ ). This Euclidean evolution generates the empty wormhole. We can then create excitations by acting by operators during the euclidean evolution period. To simplify the notation we will denote by $\varphi$ the rescaled Euclidean time $\varphi \equiv \frac{2 \pi}{\beta} u_{e}$, so that $\varphi \sim \varphi+2 \pi$. 


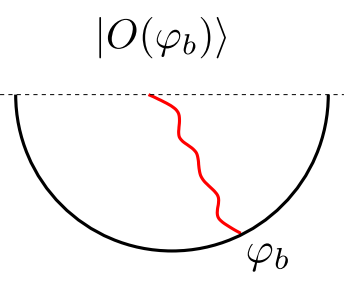

(a)

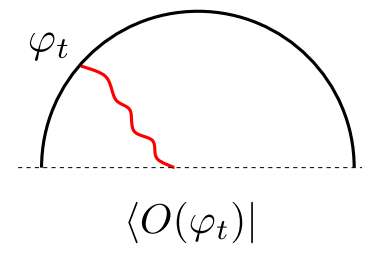

(b)

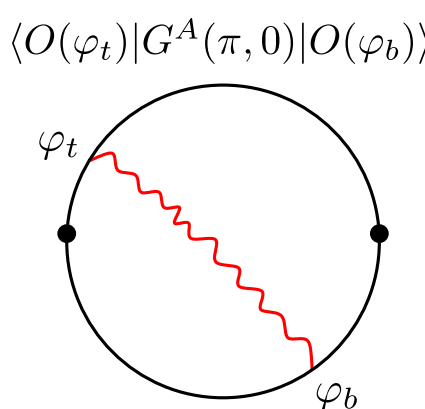

(c)

Figure 3. (a) By performing euclidean evolution over time $\beta / 2$ and inserting an operator at some point during the euclidean evolution we create a state. This defines a map between operators and states. These are states of a wormhole or states living in the Hilbert space of two copies of the dual boundary quantum system. (b) The same for the bra. (c) We can take the inner product and add the action of a charge $G^{A}(\pi, 0)$, represented by the black dots. In the semiclassical regime, these charges act as $\mathrm{SL}(2)$ generators on the states or the operators.

We can now consider a general two point function between an operator inserted on the top half of the circle and one on the bottom half

$$
\left\langle O\left(\varphi_{t}\right) O\left(\varphi_{b}\right)\right\rangle=\left\langle O\left(\varphi_{t}\right) \mid O\left(\varphi_{b}\right)\right\rangle \propto \frac{\mathfrak{s}^{2 \Delta}}{\left[\sin \frac{\varphi_{t}-\varphi_{b}}{2}\right]^{2 \Delta}}
$$

We can view this as the overlap of two states. One is a state that is obtained by doing the path integral over the bottom half and the other is the one obtained by doing the path integral over the top half. This defines an operator/state map. See figure 3.

We will now act with the charges $G^{A}(0,0)$, or in this case $G^{A}(\pi, 0)$ and will demonstrate that they act as expected on the states created by these operator insertions, in other words

$$
\left\langle O\left(\varphi_{t}\right)\left|G^{A}(\pi, 0)\right| O\left(\varphi_{b}\right)\right\rangle=\left[\zeta^{A}\left(\varphi_{b}\right) \partial_{\varphi_{b}}+\Delta\left(\partial_{\varphi_{b}} \zeta^{A}\left(\varphi_{b}\right)\right)\right]\left\langle O\left(\varphi_{t}\right) \mid O\left(\varphi_{b}\right)\right\rangle
$$

where $\zeta^{A}$ is a linear combination of the vectors generating the infinitesimal SL(2) reparametrizations (4.10), see (4.13). This is physically saying that the action of $G^{A}(\pi, 0)$ is acting with an infinitesimal reparametrization on the bottom part, see figure $3(\mathrm{c})$. We can equally view it as acting with (minus) the reparametrization on the top part, since acting with the reparametrization both on the top and bottom leaves the correlator (4.11) invariant.

We will demonstrate (4.12) as follows. First we write down the three generators and their associated vectors.

$$
\begin{array}{lll}
\tilde{B} \simeq \mathfrak{s}\left[\epsilon^{\prime}(0)+\epsilon^{\prime \prime \prime}(0)-\epsilon^{\prime}(\pi)-\epsilon^{\prime \prime \prime}(\pi)\right], & \zeta^{\tilde{B}}=1 \\
\tilde{P} \simeq \mathfrak{s}\left[\epsilon^{\prime \prime}(0)+\epsilon^{\prime \prime}(\pi)\right], & & \zeta^{\tilde{P}}=-\sin \varphi \\
\tilde{E} \simeq \mathfrak{s}\left[\epsilon^{\prime \prime \prime}(0)+\epsilon^{\prime \prime \prime}(\pi)\right], & & \zeta^{\tilde{E}}=\cos \varphi
\end{array}
$$




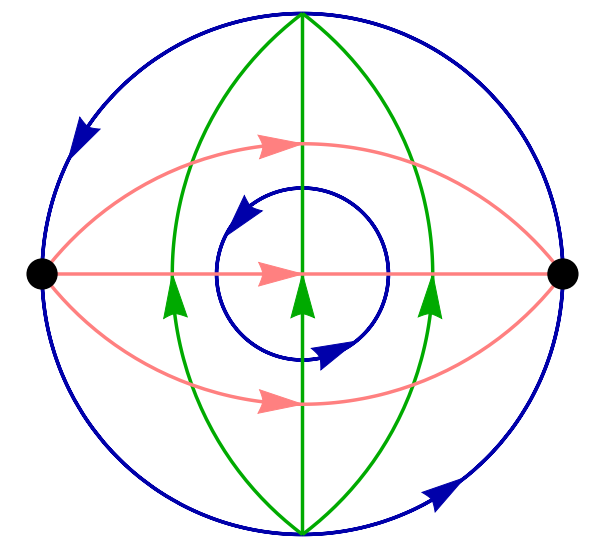

Figure 4. Geometric action of the generators in Euclidean $\mathrm{AdS}_{2}$. Here the charges are inserted at the black points, at $\varphi=0$ and $\varphi=\pi$. Blue lines follow the boost Killing vectors $\tilde{B}$; pink lines, the momentum $\tilde{P}$; and green lines, the global energy vectors $\tilde{E}$.

These are the expressions appropriate for Euclidean time. ${ }^{8}$ We can picture the geometric action of these generators as in figure 4 .

Notice that in this classical limit $\tilde{B}$ is proportional to $H_{r}-H_{l}$, see (4.4) (4.3) and it generates shifts in $\varphi$. Now, in order to evaluate the left hand side of (4.12) we will use the first order expression for $G^{A}$ in (4.13). We then also expand the correlators to first order in $\epsilon$. In other words, we write the correlators as in (2.25) and expand the times as in (4.2) to obtain

$$
\left\langle O\left(\varphi_{t}\right) O\left(\varphi_{b}\right)\right\rangle \rightarrow\left\langle O\left(\varphi_{t}\right) O\left(\varphi_{b}\right)\right\rangle\left\{1+\Delta\left[\epsilon_{t}^{\prime}+\epsilon_{b}^{\prime}-\frac{\left(\epsilon_{t}-\epsilon_{b}\right)}{\tan \frac{\varphi_{t}-\varphi_{b}}{2}}\right]\right\}
$$

where the subindices of $\epsilon$ indicate where they are evaluated, $\epsilon_{t}=\epsilon\left(\varphi_{t}\right)$, etc. Then the computation of (4.12) boils down to a computation in the linearized Schwarzian theory with action

$$
S_{E}=\mathfrak{s} \int d \varphi \frac{1}{2}\left(\epsilon^{\prime \prime 2}-\epsilon^{\prime 2}\right)+\cdots
$$

We see that the classical limit is indeed large $\mathfrak{s}$. The propagator associated to this action is [3]

$$
\langle\epsilon(\varphi) \epsilon(0)\rangle=\frac{1}{\mathfrak{s}}[G(|\varphi|)+a+b \cos \varphi], \quad G(\varphi) \equiv-\frac{(\varphi-\pi)^{2}}{4 \pi}+\frac{(\varphi-\pi)}{2 \pi} \sin \varphi
$$

where $a$ and $b$ are constants that drop out when we compute $\operatorname{SL}(2)$ gauge invariant quantities, such as the ones we are computing. So we can set them to zero. For example, to compute an insertion of $\tilde{P}$ we need to compute the correlator

$$
\frac{\left\langle O\left(\varphi_{t}\right)|\tilde{P}| O\left(\varphi_{b}\right)\right\rangle}{\left\langle O\left(\varphi_{t}\right) \mid O\left(\varphi_{b}\right)\right\rangle}=\Delta \mathfrak{s}\left\langle\left[\epsilon_{t}^{\prime}+\epsilon_{b}^{\prime}-\frac{\left(\epsilon_{t}-\epsilon_{b}\right)}{\tan \frac{\varphi_{t}-\varphi_{b}}{2}}\right]\left[\epsilon^{\prime \prime}(0)+\epsilon^{\prime \prime}(\pi)\right]\right\rangle
$$

\footnotetext{
${ }^{8}$ Relative to (4.3) we have flipped the signs of $\epsilon_{l}$ and of $u_{l}$. This arises due to a different definition of the left time. In addition, when we go from Lorentzian to Euclidean time we need to say that $\epsilon \rightarrow i \epsilon, u \rightarrow i u$. We also removed an extra $i$ in $\tilde{P}$.
} 


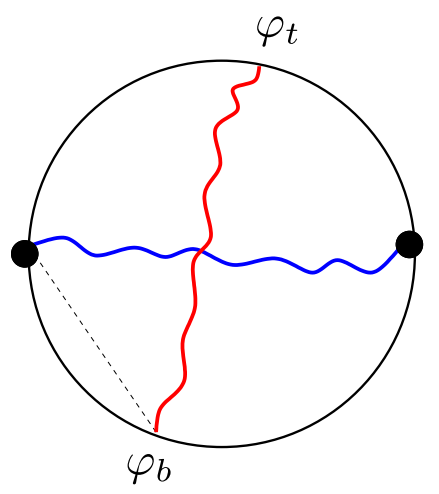

(a)

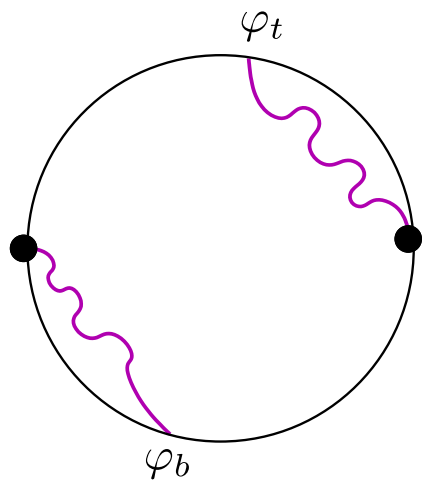

(b)

Figure 5. (a) We consider the action of the charges. We have matter fields propagating from the bottom to the top indicated in red. These cause some backreaction on the boundary positions. These are summarized by the coupling to $\epsilon$ at the insertion points of operators. The definition of the charges involves computing a distance, which implicitly, or more explicitly (in the generators $\hat{G}^{A}$ in (4.23), (4.29), (4.31)), involve the propagation of other matter fields. The interesting terms come from correlators between these $\epsilon$ insertions. We only have two point functions of $\epsilon$, only one of which is indicated in the diagram by a doted line. Other diagrams contain a dotted line between black points and $\varphi_{t, b}$. (b) In some specific models we might get contractions between the fields in the definition of the charges and the insertions. We want to suppress this type of diagrams. They are indeed suppressed relative to those in (a) in the SYK model.

Using the propagator (4.16) we find that this is equal to the expression we need to generate the right hand side of (4.12). In other words, it is

$$
\frac{\left\langle O\left(\varphi_{t}\right)|\tilde{P}| O\left(\varphi_{b}\right)\right\rangle}{\left\langle O\left(\varphi_{t}\right) \mid O\left(\varphi_{b}\right)\right\rangle}=\Delta\left[\frac{1}{\tan \frac{\varphi_{t}-\varphi_{b}}{2}} \zeta^{\tilde{P}}\left(\varphi_{b}\right)+\left(\zeta^{\tilde{P}}\left(\varphi_{b}\right)\right)^{\prime}\right], \quad \zeta^{\tilde{P}}=-\sin \varphi
$$

with $\zeta^{\bar{P}}=-\sin \varphi$, as in (4.13). The diagrams we need to compute can be seen in figure $5($ a). For $\tilde{E}$ and $\tilde{B}$ we also get results consistent with (4.12), (4.13).

In computing the matrix elements of $G^{A}$, we ignored 1-loop corrections to the 2-pt function. This is justified because such corrections actually cancel, since the zero-th order term in $G^{A}$ is actually zero. We also ignored the 1-loop correction to $G^{A}$ itself. If we use the exact charges, this too must vanish because $G^{A}$ exactly annihilates a state without matter. However, if we used approximate expressions (as we will discuss in section 4.3) for $G^{A}$, one should in principle subtract off these contributions order by order in perturbation theory, see equation (4.29).

As a more specific example we can consider the expectation values of all three generators on a state created by inserting the operator in Euclidean time at $\varphi_{b}<0$, see figure (6): We simply evaluate expressions like (4.18) setting $\varphi_{t}=-\varphi_{b}$ and we obtain

$$
\langle\tilde{P}\rangle=0, \quad\langle\tilde{B}\rangle=\frac{\Delta}{-\tan \varphi_{b}}, \quad\langle\tilde{E}\rangle=\frac{\Delta}{-\sin \varphi_{b}}
$$




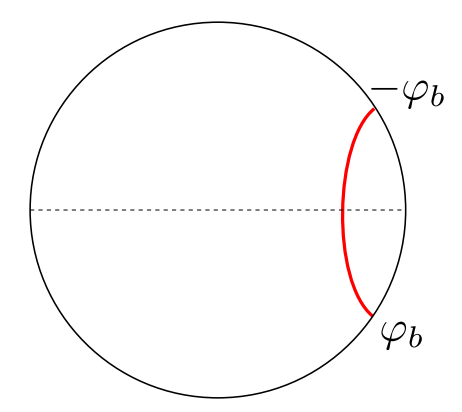

Figure 6. Inserting an operator in Euclidean time creates a particle at rest.

We should think of a state which contains a particle at rest on the initial slice. At small $\left|\varphi_{b}\right|$, the particle is located at a propert distance of the order $-\log \left(-\varphi_{b}\right)$ from the horizon, and the redshift difference between the horizon and its position if of order $1 /\left(-\varphi_{b}\right)$. See figure 6 .

The conclusion of this discussion is that around these classical states, the exact generators $G^{A}(0,0)$ are acting as $\operatorname{SL}(2)$ generators transforming the boundary time.

The primary states and their descendants defined by the state-operator correspondence are eigenstates of $\tilde{E}$ in the semiclassical approximation, but this is not expected to be exact in the Schwarzian theory. Presumably the exact eigenstates of $\tilde{E}$ could be obtained by smearing the primary in some suitable fashion.

In previous sections we have seen that $G^{A}$ maps physical states to physical states. We have seen here that this map changes states as we expect from $\operatorname{SL}(2)_{u}$ symmetries of the boundary time $u$ (4.10). In other words, the generators $G^{A}$ that are always well defined, become the $\mathrm{SL}(2)_{u}$ generators of a $\mathrm{NCFT}_{1}$ in this limit. This correspondence is not expected to hold away from the semiclassical limit. In fact, the boundary dynamics is not invariant under $\mathrm{SL}(2)_{u}$. But this is a good approximate symmetry in this classical limit. Note that the semiclassical limit is really hardwired in our description of the symmetry itself, since the action of the approximate symmetry depends explicitly on $\beta$ (4.10). This represents a state dependence of the symmetry action. And it is reflected in the dependence of the generators on $\beta$ (4.13) (and will be more explicitly seen below). Furthermore, in section 4.2.2, we will see that, as we insert matter at early lorentzian times, this semiclassical picture also breaks down.

Finally, we would like to caution the reader that this physical $\mathrm{SL}(2)_{u}$ should not be confused with the exact gauge symmetry $\mathrm{SL}(2)_{g}$ that has previously been discussed (and which we review in section 2.3). The $\mathrm{SL}(2)_{u}$ generators act on physical states $|O(u)\rangle$ and give new physical states, e.g., $\tilde{B}\left|O\left(\phi_{b}\right)\right\rangle \approx\left|\partial O\left(\phi_{b}\right)\right\rangle$.

\subsubsection{Inserting matter at early lorentzian times}

In the previous section we have discussed that inserting operators in euclidean time gives us states at $u_{l}=u_{r}=0$ that contain bulk excitations, and we explained how to read off the SL(2) charges of these states in the semiclassical limit.

Of course, these formulas also work in Lorentzian signature. More specifically, imagine that we start with the thermofield double state at early times, say $u_{l}=0, u_{r} \ll 0$, which 


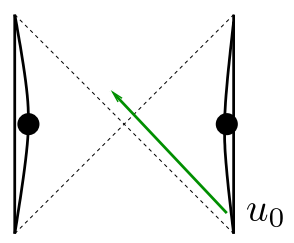

(a)

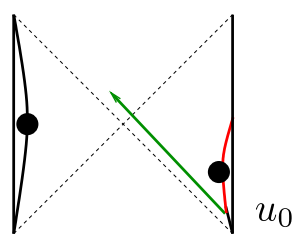

(b)

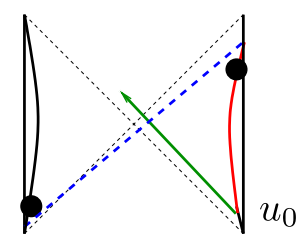

(c)

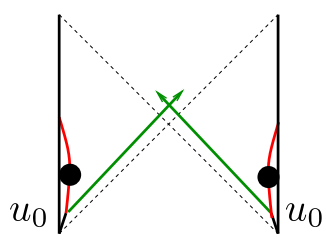

(d)

Figure 7. (a) We insert matter at some early Lorentzian time $u_{0}<0$ smaller than the scrambling time so that the backreaction on the boundary trajectory is small. (b) Same insertion but beyond the scrambling time. There is a large change in the boundary trajectory. (c) The same as (b) but after an overall boost (a gauge transformation) that explains better why there is a maximum momentum. Here we have kept the point $u_{0}$ fixed in the figure so that increasing it corresponds to moving the left and right dots (the times where we evaluate the charges) down and up. The point is that the boost angle between the green line (the matter we inserted) and the blue dotted line is finite. The blue dotted line represents that highest boost angle for a geodesic joining the two black dots at late times. (d) We insert matter on both sides. In this case, one can show that the energy continues to increase as we take $u_{0}$ beyond the scrambling time.

we can obtain by evolving the TFD state backwards in time on one of the sides. We then insert an operator at time $u_{r 0}<0$, and evolve up to $u_{r}=0$. See figure 7(a). We will need to slightly smear it in order to create a relatively low energy state that can be described within the conformal regime. This will also create a matter state inside the wormhole. We can find the SL(2) transformation properties of this state by acting with the charges, and we will obtain the expected action, as indicated in (4.12). It is interesting that now some of the "conformal Killing vectors" have an exponential depedence on time,

$$
\zeta^{\tilde{B}}=1, \quad \zeta^{\tilde{P}}=\sinh \frac{2 \pi u}{\beta}, \quad \zeta^{\tilde{E}}=\cosh \frac{2 \pi u}{\beta}
$$

This implies that if we insert the same operator $O$, earlier and earlier in time, we will get exponentially growing values for its energy and its momentum, from (4.12). At least this is true as long as these semiclassical expressions hold. It turns out that for early times, times larger than the scrambling time, $u_{\mathrm{scr}}=\frac{\beta}{2 \pi} \log \mathfrak{s}$, it becomes important to take into account the backreaction beyond the leading order in $\epsilon$.

\subsubsection{Inserting matter beyond the scrambling time and corrections to the semiclassical limit}

We have seen in the previous subsection that if we insert some mode of energy $\omega$ at some early time $u_{0}$, then its charges evaluated at $u_{l}=u_{r}=0$ grow exponentially as $\beta \omega e^{-\tilde{u}_{0}}$ $\left(\tilde{u}_{0}=\mathfrak{s} u_{0}\right.$ is negative). Then, even if $\mathfrak{s} \gg 1$, there can be a time when the simple small $\epsilon$ approximation breaks down. The expansion parameter is really $\beta \omega e^{-\tilde{u}_{0}} / \mathfrak{s}$, and the small $\epsilon$ approximation breaks down when this is of order one. This is the so called scrambling time [27]. The picture is that, by this time, the excitation has an order one commutator with any other simple excitation. Now, our basic expressions for the generators are exact and can be evaluated beyond the scrambling time. In this section we sketch the results for the exact generators (3.1) (3.2) when we go beyond the scrambling time. We will work 
in the large $\mathfrak{s}$ approximation, and for simplicity we will further assume that $\omega / \mathfrak{s} \ll 1$ and $\left|u_{0}\right| \ll 1$ but we will work exactly in

$$
\alpha \equiv \frac{\beta \omega}{\mathfrak{s}} e^{-\tilde{u}_{0}}, \quad \tilde{u}_{0}=\mathfrak{s} u_{0}=\frac{2 \pi u_{0}}{\beta}
$$

In this regime, we can find the correction to the classical trajectory and compute the generators, see figure 7(b). We find that the generators are equal to, see appendix D.1,

$$
\tilde{P} \sim \tilde{E} \sim 2 \mathfrak{s} \frac{\hat{\alpha}}{1+\hat{\alpha}}, \quad \tilde{B} \sim 0, \quad \hat{\alpha} \propto \frac{\omega \beta}{\mathfrak{s}} e^{-\tilde{u}_{0}}
$$

where $\hat{\alpha}$ is equal to $\alpha$ in (4.21), up to a numerical constant. It is also worth noting that $X_{l} \cdot X_{r} \propto(1+\hat{\alpha})^{2}$. This implies that the physical distance, which is the logarithm of this quantity increases linearly with $u_{0}$, as $\ell \sim 2\left|\tilde{u}_{0}\right| \sim 4 \pi\left|u_{0} / \beta\right|$. The standard semiclassical expression discussed in section 4.2 amounts to expanding (4.22) to first order in $\hat{\alpha}$. Interestingly we find that the generators saturate at an amount of order $\mathfrak{s}$ which is independent of the energy of the particle we have sent in. This might seem surprising, since it naively looks like we are inserting a higher and higher energy state as we take $u_{0} \rightarrow-\infty$. However, this insertion is moving the dynamic right boundary and is changing the notion of momentum. Notice that the bulk Casimir is zero in this limit, see figure $7(b, c)$. The saturation of (4.22) will be related to the decay of out of time order correlators in section 4.4.

We could consider a different experiment where we send matter from both sides, see figure 7 (c). In this case, $\tilde{P} \sim \tilde{B} \sim 0$, but $\tilde{E}$ continues to increase exponentially.

This computation illustrates how the exact generators (3.1) and (3.2) can be defined and used, beyond the scrambling time.

\subsection{Other semiclassical expressions for the generators}

We have seen that we can get approximate expressions for the SL(2) generators. These approximate expressions relied purely on the small $\epsilon$ expansion of the boundary trajectories around a given thermofield double state. Here we want to relate these expressions to correlators in the boundary theory. Of course, we have already given an expression of the exact generators in terms of the distances that are probed by boundary correlators, (3.6) (3.9). Here we want to provide simple expressions that give the same answer in the semiclassical limit.

We have already mentioned one of them. Namely, the boost generator can be approximately given in terms of the difference of Hamiltonians

$$
\tilde{B} \simeq \hat{B}=\frac{\beta}{2 \pi}\left(H_{r}-H_{l}\right)
$$

This is also the modular Hamiltonian that arises when we split the system into left and right sides. Note that $\hat{B}$ is an exact symmetry of the thermofield double state. 
The paper [17] discussed a coupled system whose Hamiltonian could be viewed as the global time translation, $\tilde{E}$, in $A d S_{2}$. This Hamiltonian was defined as

$$
\begin{aligned}
H_{\text {coupled }} & =H_{r}+H_{l}-\tilde{\eta} \sum_{j} O_{l}^{j} O_{r}^{j} \\
& \sim H_{r}+H_{l}-\eta\left(\frac{t_{l}^{\prime} t_{r}^{\prime}}{\cosh ^{2} \frac{t_{l}+t_{r}}{2}}\right)^{\Delta}, \quad \eta=\tilde{\eta} N 2^{-2 \Delta}
\end{aligned}
$$

where we have indicated the approximate expression in the Schwarzian theory in the approximation that the effect of the boundary coupling on the bulk matter is very small. We normalized the operators so that they go like $\left\langle O_{r}\left(u_{1}\right) O_{r}\left(r_{2}\right)\right\rangle \sim\left|u_{12}\right|^{-2 \Delta}$ at short distances. Then the main effect of the coupling is on the Schwarzian variables [17]. We expand around a solution of the form

$$
t_{l}=t_{r}=\mathfrak{s} u
$$

The solution that minimizes the energy (and obeys all necessary equations of the two Schwarzian theories) is such that

$$
\mathfrak{s}^{2-2 \Delta}=\Delta \eta
$$

Since the semiclassical limit involves $\mathfrak{s} \gg 1$, we need that $\eta \gg 1$. This can be achieved by having a large number of operators in (4.24). In other words, we take small $\tilde{\eta}$, but large $N$, so that $\eta$ in (4.24) is large. In the construction of [17] this equation, (4.27), was viewed as determining $\mathfrak{s}$, or $\beta$, in terms of $\eta$. The ground state of the system is close to the thermofield double at inverse temperature

$$
\beta=\frac{2 \pi}{\mathfrak{s}}
$$

This is not the physical temperature of the coupled system, it is rather the effective temperature of the density matrix of each side on its own. Finally, the normalized global time translation symmetry is then

$$
\begin{aligned}
\tilde{E} \simeq \hat{E} & \equiv \frac{1}{\mathfrak{s}}\left[H_{\text {coupled }}-\left\langle H_{\text {coupled }}\right\rangle_{0}\right] \\
\hat{E} & \simeq-\mathfrak{s}\left(\epsilon_{l}^{\prime \prime \prime}+\epsilon_{r}^{\prime \prime \prime}\right)
\end{aligned}
$$

where the last expression agrees with (4.3), as expected. Here $\langle\cdots\rangle_{0}$ indicates the expectation value in the ground state of the coupled system.

For the purposes of this paper, we can simply view the TFD state at a given inverse temperature, $\beta$, as given. And we then write (4.29), solving for $\eta$ in terms of $\mathfrak{s}=\frac{2 \pi}{\beta}$ via (4.27), and construct $\hat{E}$ as in (4.29). The advantage of this procedure is that it gives an approximate expression for $\tilde{E}$ that is relatively simple, we only need to couple the two sides. 
Finally, we can get a simple expression for $\tilde{P}$ by taking the commutator of (4.23) and (4.29), to obtain

$$
\begin{aligned}
\tilde{P} \simeq \hat{P} & \equiv-i[\hat{B}, \hat{E}]=\frac{-i}{\mathfrak{s}}\left[H_{r}-H_{l},-\tilde{\eta} \sum_{j} O_{l}^{j} O_{r}^{j}\right]=\frac{\tilde{\eta}}{\mathfrak{s}^{2}} \sum_{j}\left(O_{l}^{j} \dot{O}_{r}^{j}-\dot{O}_{l}^{j} O_{r}^{j}\right) \\
& \simeq \frac{1}{\mathfrak{s}^{2}}\left(\partial_{u_{r}}-\partial_{u_{l}}\right) \eta\left(\frac{\dot{t}_{l} \dot{t}_{r}}{\cosh ^{2} \frac{t_{l}+t_{r}}{2}}\right)^{\Delta} \\
\hat{P} & \simeq \mathfrak{s}\left(\epsilon_{r}^{\prime \prime}-\epsilon_{l}^{\prime \prime}\right)
\end{aligned}
$$

where we have used (4.27).

Notice that the generators $\hat{B}, \hat{E}, \hat{P}$ are completely well defined if the system has a quantum mechanical dual. For example, they are well defined in the SYK model. However they do not obey an exact $\mathrm{SL}(2)$ algebra. In addition, their definition depends on $\beta$ (via $\mathfrak{s})$. This means that they behave as $\operatorname{SL}(2)$ generators only for states close to the thermofield double state with that inverse temperature. The fact that they obey the right algebra for such states comes from their connection to the matter charges in (4.3). Notice that the thermofield double state, or empty wormhole, really comes in a two parameter family, parametrized by the temperature and a relative time shift between the two sides, see $[28,29]$. Again, these generators act as desired only for a particular synchronization of the two times. This is implicit in the above formulas when we write left-right correlators "at the same time".

\subsection{Order from chaos}

We can wonder what happens if we take the generators we defined, which are defined in terms of correlators at $u_{l}=u_{r}=0$ and we "evolve" them with the boost Hamiltonian. We then get, in Lorentzian time,

$$
\begin{aligned}
& e^{i \tilde{u} \hat{B}} \hat{E} e^{-i \tilde{u} \hat{B}}=\frac{\beta}{2 \pi}\left[H_{r}+H_{l}-\tilde{\eta} \sum_{j} O_{l}^{j}(-u) O_{r}^{j}(u)-\langle\cdots\rangle_{\mathrm{TFD}}\right] \simeq \cosh \tilde{u} \hat{E}-\sinh \tilde{u} \hat{P} \\
& e^{i \tilde{u} \hat{B}} \hat{P} e^{-i \tilde{u} \hat{B}}=\partial_{\tilde{u}}\left(\frac{\beta \tilde{\eta}}{2 \pi} \sum_{j} O_{l}^{j}(-u) O_{r}^{j}(u)\right) \simeq-\sinh \tilde{u} \hat{E}+\cosh \tilde{u} \hat{P}
\end{aligned}
$$

where $\langle\cdots\rangle_{T F D}$ indicates the expectation value of the previous three terms in the TFD state. The first equality is what we get from the explicit definition of the hatted generators. The second equality is expected to hold for states that are close to the thermofield double, and it holds to the extent that we can approximate the hatted generators by the matter ones in the semiclassical limit, see (4.3) and to the extent that the hatted operators obey an approximate $\mathrm{SL}(2)$ algebra.

We can think of (4.33) as an approximate expression for the approximate symmetries at zero time in terms of operators at other times.

Notice that in (4.33) we have exponentially growing terms in the right hand side as $\tilde{u} \rightarrow \infty$. Such terms can only come from the term involving $O_{l}^{j}(-u) O_{r}^{j}(u)$, which indeed 


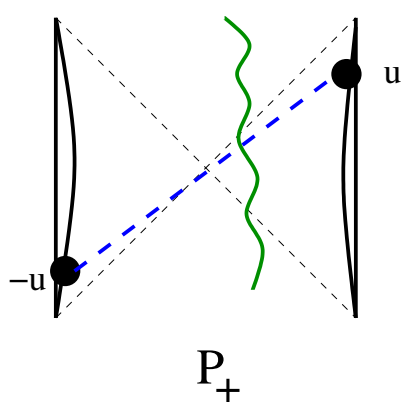

(a)

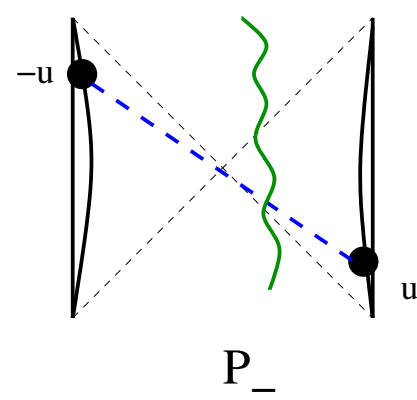

(b)

Figure 8. (a) In order to measure $P_{+}$we can consider a correlator in this configuration with large $u$, but smaller than the scrambling time. (b) To measure $P_{-}$we consider instead a correlator at these times.

can lead to exponential growth. The reason is the following. The expectation values of these operators on a state created by acting with operators on the thermofield double is an out of time order correlator. This is an analytic continuation to Lorentzian time of a configuration of operators as in figure 5(a). In (4.33) we are computing the difference between this out of time order correlator and the disconnected correlator contained in the thermofield double expectation value $\left\langle O_{l}^{j}(-u) O_{r}^{j}(u)\right\rangle_{\mathrm{TFD}}$. The latter is time independent due to the boost symmetry of the empty wormhole or thermofield double. On the other hand the out of time order correlator decays as $u$ increases [30, 31]. This initial decay is given by an exponentially growing deviation from the disconnected diagram [30, 31]. Since we have a difference between the two correlators in (4.33), we only pick up the correction that is exponentially growing in time.

We can concentrate on these growing terms and write a simple expression for $P_{ \pm}$at time equal to zero in terms of correlators at other times

$$
\begin{aligned}
& -P_{+}=\frac{\hat{E}-\hat{P}}{2}=-\lim _{u \rightarrow+\text { large }} e^{-\frac{2 \pi u}{\beta}} \frac{\beta \tilde{\eta}}{2 \pi} \sum_{j}\left[O_{l}^{j}(-u) O_{r}^{j}(u)-\left\langle O_{l}^{j}(-u) O_{r}^{j}(u)\right\rangle_{\mathrm{TFD}}\right] \\
& -P_{-}=\frac{\hat{E}+\hat{P}}{2}=-\lim _{u \rightarrow-\operatorname{large}} e^{\frac{2 \pi u}{\beta}} \frac{\beta \tilde{\eta}}{2 \pi} \sum_{j}\left[O_{l}^{j}(-u) O_{r}^{j}(u)-\left\langle O_{l}^{j}(-u) O_{r}^{j}(u)\right\rangle_{\mathrm{TFD}}\right]
\end{aligned}
$$

where $\tilde{\eta}$ is fixed by (4.25) and (4.27). The explicit exponential prefactors are decreasing in the corresponding limits and extract the growing pieces of the correlator corrections. In these equations when we say "large" we mean a large time but smaller than the scrambling time. In other words, a time obeying

$$
1 \ll \tilde{u} \ll \log \mathfrak{s}
$$

Therefore, these formulas make sense only in the semiclassical limit, where $\mathfrak{s} \gg 1$.

The growing nature of the left-right correlators in the presence of matter is related to chaos [30,31]. It was found that this growth is related to gravitational shockwaves which inducing null shifts of the bulk matter. Here we are inverting the logic and using 
these growing pieces to define the action of the generators. In this sense we are getting a symmetry (order) from chaos.

Alternatively, it was shown in [16] (see also [32]) that the two sided correlators induce null displacements of the matter propagating inside, when there is a large relative boost between the two. This is related to the phenomenon of quantum teleportation. Here we are using this phenomenon to talk about the symmetries.

In fact, the present discussion suggests that we will be able to use this growth for any non-zero temperature black hole, not just near extremal ones. The only difference will be in the value of the commutator between $\left[P_{+}, P_{-}\right]$. In our case this gives $B$. But for a generic black hole we expect that this should be zero, because the symmetry near any horizon is just Poincare. In fact, even in our case, if we consider excitations that are very close to the horizon, they will have large values of $P_{+}$and $P_{-}$so that is natural to rescale the generators, making them smaller. This in turn will also rescale the commutator. On the other hand, near any black hole horizon the boost generator has a natural universal normalization which is that of the "modular" Hamiltonian (conjugate to standard Rindler time).

Finally, we should remark that by looking at (4.33), which was derived from algebraic and symmetry considerations, we can deduce that the expectation value of $\sum_{j} O_{l}^{j}(-u) O_{r}^{j}(u)$, in a perturbed thermofield double state, should contain a term growing exponentially with maximal Lyapunov exponent in order to match the right hand side of (4.33). So we can view this as an algebraic derivation of the maximal chaos behavior. Of course, this is essentially the same as the original gravitational derivation using shock waves $[30,31]$ after we use the particular features of nearly $\mathrm{AdS}_{2}$ gravity.

\subsection{Generators for the one-sided case}

All of the charges we have been discussing use two-sided operators. To what extent can we define physical matter charges if we only have access to one side? Clearly it is impossible to determine the matter charges for a general state with only one-sided observables. However, it is plausible that we could detect the matter charges on restricted states, where matter is inserted only from one side.

Let us be more concrete. The generators in section 3.1 were of the form $G^{A}=-e_{a}^{A}\left(Q_{l}^{a}+Q_{r}^{a}\right)$. Below we will consider choices of $e^{A}$ that only depend on the right side. Nevertheless, the presence of $Q_{l}^{a}$ seems troublesome. Now imagine that we start with a state with no matter, e.g., as $u \rightarrow \infty$, the matter charge vanishes $Q_{m}^{a}=0$. On such states $Q_{l}^{a}(-\infty)=-Q_{r}^{a}(-\infty)$. If we furthermore assume that the left boundary evolves with the standard Hamiltonian $H_{l}$ with no matter insertions, then $Q_{l}^{a}$ is conserved for all times, so we may write

$$
Q_{l}^{a}=-Q_{r}^{a}(-\infty)
$$

We may also write this as

$$
\Delta G_{o s}^{A}=-e_{a}^{A} \Delta Q_{r}^{a}, \quad \Delta Q_{r}^{a}=Q_{\mathrm{r}, \text { after }}^{a}-Q_{\mathrm{r}, \text { before }}^{a}
$$

$\Delta Q_{r}^{a}$ measures the change in the right gauge charges before and after the matter insertion. The subscript "os" means one sided. Note that we are really defining the change in the generators, not the generators themselves. 


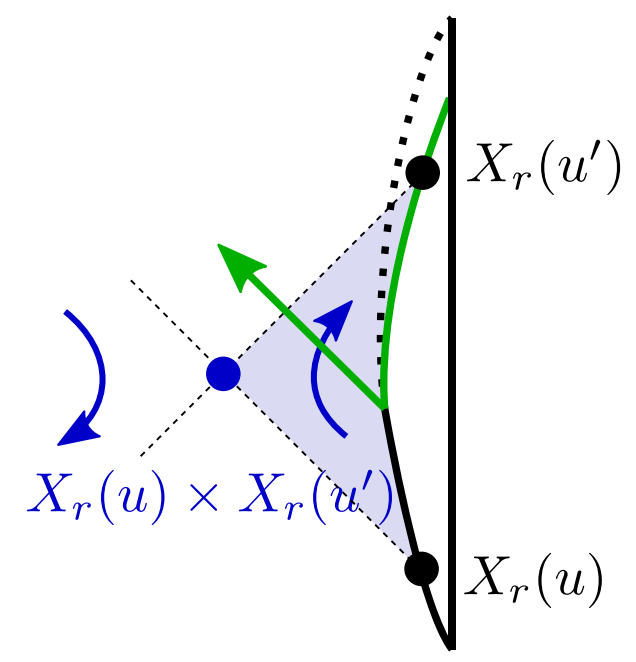

Figure 9. The one-sided generator. An unknown amount of matter is inserted at some time between $u$ and $u^{\prime}$. Our task is to detect it using right-sided observables. The blue point in the center is proportional to $X_{r}(u) \times X_{r}\left(u^{\prime}\right)$. The generator corresponding to this point fixes the causal wedge (shaded in light blue).

We now consider various choices of $e^{A}$. A relatively natural one is to use $X_{r}^{a}$ at two different times $X_{r}(u), X_{r}\left(u^{\prime}\right)$. For the rest of this subsection, all quantities will be on the right side, so we will drop subscripts.

$$
e_{a}^{A}=\left(e_{a}^{-1}, e_{a}^{+}, e_{a}^{-}\right)=\left(\frac{\left(X_{r} \times X_{r}^{\prime}\right)_{a}}{X_{r} \cdot X_{r}^{\prime}}, \sqrt{2} \frac{X_{r_{a}}(u)}{\sqrt{X_{r} \cdot X_{r}^{\prime}}}, \sqrt{2} \frac{X_{r a}\left(u^{\prime}\right)}{\sqrt{X_{r} \cdot X_{r}^{\prime}}}\right)
$$

The generators associated to such vectors have a nice geometric interpretation in terms of causal wedges. Namely, if $u<u^{\prime}$ then we shoot a future directed light ray from $u$ and past directed light ray from $u^{\prime}$. Then the "causal wedge" is defined to be what is enclosed by these light rays, see figure 9 . The first vector in (4.38) gives a generator that performs a boost around the intersection of the light rays, see figure 9. This boost generator maps points in the causal wedge to points in the causal wedge. In the QFT approximation to the bulk physics, ignoring gravity, one can view it as the modular Hamiltonian of the causal wedge. ${ }^{9}$

This construction is also closely related to the recent work of [33]. There, they associate to two boundary times $u$ and $u^{\prime}$ the point $X_{r}(u) \times X_{r}\left(u^{\prime}\right)$. They consider a bulk operator at such a point. In other words, they gravitationally dress a bulk matter fields with the gravitational operators $X_{r}(u) \times X_{r}\left(u^{\prime}\right)$ in order to define a diffeomorphism invariant operator. Here we are dressing the matter charge with the same gravitational operators to obtain $G_{o s}^{-1}$.

One problem with the vectors in (4.38) is that they fail to commute in the quantum theory. To quantify the extent of this problem, let us compute the commutator of the last two vectors in the semiclassical limit. We can do this by writing the general classical

\footnotetext{
${ }^{9}$ In the full gravity theory the causal wedge should be only an approximate notion that arises when we restrict to simple operators in the boundary theory.
} 
solution

$$
X^{a}=-\frac{Q^{a}}{2 H}+\left(X^{a}(0)+\frac{Q}{2 H}\right) \cosh (\sqrt{2 H} u)+\frac{\dot{X}^{a}(0)}{\sqrt{2 H}} \sinh (\sqrt{2 H} u)
$$

We can then compute the Poisson bracket $\left\{X^{a}(u), X^{b}(0)\right\}_{P B}$. There will be many terms; the important point is that

$$
\left\{X^{a}(u), X^{b}(0)\right\}_{P B}=M^{a b} e^{\sqrt{2 H} u}+\cdots,
$$

where $M^{a b}$ is a function of $X(0), \dot{X}(0), H$, but independent of $u$. The important point is that there is an exponentially growing contribution to the commutator. On a thermal state, $\lambda=\sqrt{2 H}=2 \pi / \beta$; this is exactly the maximal Lyapunov exponent. It is somewhat ironic that the maximal chaos, which we said was useful for constructing the two sided generators, is also what prevents us from defining good 1-sided charges.

This motivates us to consider $u \rightarrow u^{\prime}$ so that the commutator is smaller. This is equivalent to choosing

$$
e_{a}^{A}=\left(\dot{X}_{a}, \frac{\ddot{X}_{a}}{\sqrt{2 H}}, \frac{(\dot{X} \times \ddot{X})_{a}}{\sqrt{2 H}}\right) .
$$

Since $\dot{X} \cdot \dot{X}=-1$, the first two vectors are automatically orthogonal $\ddot{X} \cdot \dot{X}=0$. Note, however, that there will be a non-trivial commutator between the different components of $e^{A}$. For example, the commutator of the first two components will get a contribution from

$$
\left[\ddot{X}^{a}, \dot{X}^{b}\right]=i \epsilon^{a b c} \dot{X}^{c}+i\left\{H, X^{a} X^{b}\right\}-i\left\{\ddot{X}^{a}, X^{b}\right\} .
$$

Here $\{A, B\}=A B+B A$. If we contract this expression with the matter charges $\Delta Q_{m}^{a} \Delta Q_{m}^{b}$ the last two terms can become large. In particular, it grows exponentially as a function of the boundary time when the matter was inserted. So even if we use vectors at the same time, chaos will lead to the breakdown of the one-sided algebra if we wait too long after perturbing the right side.

Finally, let us turn to the momentum discussed in [19]. They consider the momentum of a particle thrown in from one side. "momentum" here means the variable conjugate to distance. But distance from what? It is most natural to take the distance from the bifurcating surface on the left side, since the bifurcating surface on the right changes when matter is inserted. The left bifurcating surface sits at a point $Y^{a} \propto Q_{l}^{a}$, which corresponds to a definition of momentum

$$
\tilde{P}_{\mathrm{os}}=Q_{m} \cdot \frac{Q_{l} \times X_{r}}{Q_{l} \cdot X_{r}}=\frac{\dot{X}_{r} \cdot Q_{l}}{X_{r} \cdot Q_{l}}=\frac{d}{d u_{r}} \log \left(-X_{r} \cdot Q_{l}\right) .
$$

From the last line, it is clear that $\tilde{P}_{\mathrm{oS}}$ is proportional to the velocity of the right boundary particle relative to the entangling surface. Note, as above, we may replace $Q_{l}=-Q_{r}(-\infty)$ to arrive at a purely one-sided quantity.

If we consider semiclassical states with a particle thrown in at some time $u_{0}<0$ from the right, the one-sided momentum approximates the two-sided momentum $\tilde{P}_{\text {os }} \approx \tilde{P}$ as long as $\left|u_{r}\right|$ is less than the scrambling time. This is because the geodesic connecting $X_{r}$ and $X_{l}$ approximately intercepts the bifurcating surface $Q_{l}$. 


\section{Exploring the bulk}

\subsection{Evolving with the charges}

In section 4.3 we pointed out that some generators, such a $\tilde{E}$, can be approximated, in the semiclassical limit, by a simple coupled Hamiltonian (4.24) (4.29). It is natural to ask whether it is possible to systematically correct this coupled Hamiltonian so that it gives the exact generator $\tilde{E}$.

One simple way to think about this is to declare that the full Hamiltonian of the coupled system is simply

$$
\tilde{H}_{\text {coupled }}=\tilde{E}
$$

This is not the same as (4.29), hence the tilde. This seems a legitimate Hamiltonian from the point of view of the gravity theory. ${ }^{10}$

This Hamiltonian has a number of differences with (4.24) or $\hat{E}_{c}$ in (4.29). A simple difference is that we do not need to subtract the ground state energy as in (4.29). In fact, by construcution, $\tilde{E}$ annihilates the thermofield double state. A more important difference is that $\hat{E}$ in (4.29) depends on $\beta$ (through the temperature dependence on (4.27) (4.28). This means that $\hat{E}$ is close to the generator $\tilde{E}$ only for states that are close enough to the TFD states with inverse temperature $\beta$. In contrast, the Hamiltonian (5.1) is $\beta$ independent. TFD states with any temperature and any relative synchronization between left and right times are ground states of (5.1). In (5.1) only states with nontrivial bulk matter have non-zero energy (under the Hamiltonian $\tilde{H}_{\text {coupled}}$ ).

In the presentation of the charges in (3.2), we see that $\tilde{E}$ moves the matter along the global time translation symmetry generator but leaves the boundaries at the same positions. Up to an $\mathrm{SL}(2)_{g}$ gauge symmetry this is the same as moving the boundaries and keeping the bulk matter fixed. This is close to what we mean by the time evolution of the bulk observer. The picture is very similar to the one for the evolution with (4.24), [17], where the two physical boundaries move vertically in global $A d S_{2}$. The difference is that these physical boundaries can have any location here, while under (4.24) they had a preferred location.

In order to explore the relation between $H_{\text {coupled }}$ and $\tilde{H}_{\text {coupled }}$ a bit further, it is useful to write the expression for $\tilde{E}$ in terms of the global time $T_{l}$ and $T_{r}$. In particular, if we act by physical symmetries and choose a special gauge we can classically restrict ${ }^{11}$ to symmetric configurations $T_{l}(u)=T_{r}(u)$. We find from $(3.9)$

$$
\tilde{E}=2\left(-T^{\prime}+\frac{T^{\prime \prime 2}}{T^{\prime 3}}-\frac{T^{\prime \prime \prime}}{T^{\prime 2}}\right)=-2 e^{-\varphi}\left(\varphi^{\prime \prime}+e^{2 \varphi}\right), \quad \tilde{P}=\tilde{B}=0
$$

\footnotetext{
${ }^{10}$ Non-perturbative corrections that can render the distance between the two boundaries ill defined, and therefore (5.1) ill defined. We ignore such corrections here.

${ }^{11} \mathrm{By}$ acting on a general state with the physical symmetries, we can set $\tilde{P}=\tilde{B}=0$ at $u_{l}=u_{r}=0$. We then use the $\mathrm{SL}(2)_{g}$ gauge symmetry to set $t_{l}(0)=t_{r}(0)=0$ and $t_{l}^{\prime}(0)=t_{r}^{\prime}(0)$. So the matter and physical charges align $e_{a}^{A}=\delta_{a}^{A}$, at $u_{l}=u_{r}=0$. Now the two equations $\tilde{P}(0,0)=0, \tilde{B}(0,0)=0$ set $t_{l}^{\prime \prime}(0)=t_{r}^{\prime \prime}(0)$ and $t_{l}^{\prime \prime \prime}(0)=t_{r}^{\prime \prime \prime}(0)$. So at $u_{l}=u_{r}=0$ the coordinates and momenta are equal on both sides. Then using the classical equations of motion, the coordinates and momenta are the same for all times. Hence $t_{l}(u)=t_{r}(u)$. Note that this argument could be rerun with the coupled Hamiltonian with almost no modification.
} 
with $\varphi \equiv \log T^{\prime}$. This is also the matter energy in our gauge, and this the same as the gauge constraint $E_{m}=-Q_{l}^{1}\left(T_{l}\right)-Q_{r}^{1}\left(T_{r}\right)=\tilde{E}$. To derive this formulas we can write $X_{r}^{A}=\left(\cos T_{r}, \sin T_{r}, 1\right) / T_{r}^{\prime}$ and a similar expression for $T_{l}$.

We can interpret the full expression for $\tilde{E}$ in (3.9) as follows. The prefactor

$$
\left(-2 X_{l} \cdot X_{r}\right)^{1 / 2}=2 e^{-\varphi}
$$

simply gives a redshift factor of order $\beta$ when acting on states near the thermofield double. The first three terms in parentheses of (3.9) is precisely the Hamiltonian in [17] with $\Delta=1$ and $\eta=1 / 2$

$$
H_{\text {coupled }}=-2 \varphi^{\prime \prime}+\left(\varphi^{\prime}\right)^{2}-e^{2 \varphi}-\eta e^{2 \Delta \varphi}, \quad \eta=\frac{1}{2}, \quad \Delta=1
$$

The last terms in (3.9) give a similar expression which combines to

$$
\tilde{E}=\left(-2 X_{l} \cdot X_{r}\right)^{\frac{1}{2}}\left(-\varphi^{\prime \prime}-e^{2 \varphi}\right)=2 e^{-\varphi}\left(\frac{1}{2} e^{\varphi} E_{m}\right)=E_{m}
$$

where we used (5.2) viewed as a gauge constraint. We see that the dynamical boundary variables have disappeared. So with this Hamiltonian, the boundary has no dynamics. This expression, (5.5), looks misleadingly simple because it was written in a special gauge. In order to act with $\tilde{E}$, we do need to know the boundary positions. We need to know the relative synchronization of the two times, for example, and to extract this information we need to measure some left-right correlators. This is a common feature in gauge theories, where an expression in a fixed gauge might look local (here $\tilde{E}$ appears to involve only one factor in the Hilbert space), but the full gauge invariant operator is not local.

Before proceeding, let us mention a subtlety in the above discussion. In the above, we wrote expressions which involved derivatives with respect to $u$. These expressions implicitly assumed that $u$-translation was generated by $H_{l}+H_{r}$. However, when we imagine evolving with $\tilde{E}$ or with the coupled Hamiltonian $H_{\text {coupled, }}$ our expressions will be modified. A better approach is to write this in terms of coordinates and momenta. This is developed in appendix B. There, we give explicit formulas for the charges and $X_{l, r}^{a}$ in terms of the coordinates $T, T^{\prime}$ and their conjugate momenta $p_{1}, p_{2}$ (see equation (B.7) and (B.1)). Using these formulas, we can express $\tilde{E}$ in terms of coordinates and momenta via the relation (3.2) or via (3.4).

If we denote by $p_{1}^{r}, p_{2}^{r}$ the momenta conjugate to the global times $T_{r}(u)$ and $T_{r}^{\prime}(u)$, the statement is that when coordinates and momenta for different sides are always equal (up to minus signs), then we get the simple expression

$$
T^{\prime} \tilde{E}=-T^{\prime}\left(p_{1}^{l}+p_{1}^{r}\right)
$$

Then evolving by $T^{\prime} \tilde{E}$ gives the solution $T=-T^{\prime} u$.

In [17], the low energy spectrum of the coupled wormhole was approximately a tensor product of the bulk matter $e^{\varphi} E_{m}$ and the boundary Schwarzian degrees of freedom whose breathing mode is an anharmonic oscillator. While the bulk matter Hamiltonian $e^{\varphi} E_{m}$ 
organizes into SL(2) multiplets, the boundary degrees of freedom does not, since for one thing the oscillator's frequency differs from the $\mathrm{AdS}_{2}$ frequency. Viewed as a Hamiltonian $\tilde{E}$ solves the above problem by subtracting off the kinetic terms and then flattening out the potential energy of the oscillator. Finally, there is an overall factor which removes the redshift factor so that the Hamiltonian is exactly the bulk energy. The result is an energy spectrum of $\tilde{E}$ that is independent of the Schwarzian modes. One might also imagine an opposite strategy of achieving an approximate $\mathrm{SL}(2)$ spectrum where instead of removing the energy of the boundary degrees of freedom, one makes the frequency of oscillation so large that the boundary modes are essentially frozen, and we have an effective description that only involves the SL(2) matter. A preliminary exploration of this idea is given in appendix F.

While $\tilde{H}_{\text {coupled }}$ makes sense in JT-gravity plus matter, one can question whether we can really construct it from a more microscopic theory, such as a full boundary quantum mechanical theory. This is of course a question about all $G^{A}$ generators. In the next section, we discuss a particular large $N$ scaling limit of SYK, where these generators make sense. On the other hand, in a boundary quantum mechanical theory with a finite Hilbert space, we should not be able to construct the generators $G^{A}$ (since they generate an infinite number of states). The difficulty lies in measuring distance, as we will discuss in section 7.1.

\subsection{Exploring behind the horizon or moving the horizon}

One of our motivations was to understand better how matter moves in the bulk and how that is represented in the boundary theory. The generators we constructed allow us to move matter in the bulk relative to the boundaries, so they allow us to explore the bulk. One would like to be able to explore the region behind the horizon. Indeed these charges allow us to move matter within the Wheeler-de-Witt patch, see figure 2 .

Actually, it is also important to understand the sense in which we can move matter. When we act with the generator $\tilde{E}$, for example, we are either moving matter or moving the boundaries (these are two equivalent descriptions). Let us take the point of view that we leave the matter in the bulk as it is but we move the boundaries forwards in time along the vertical direction in the Penrose diagram (i.e. by performing a global time translation $T \rightarrow T+$ constant (2.6a)). But, if after doing this, we let the boundaries evolve with decoupled Hamiltonians, then we would find now the horizon at a new position, see figure 10 (c). So, we can say that the $\tilde{E}$ generator, allows us to explore the region that would have been behind the horizon if we had done nothing. By the very act of evolving with $\tilde{E}$, we have brought it out of the horizon (see [16]). The horizon is a teleological object, and in the quantum theory, it is related to the limitation on the types of experiments we can do. For example, it depends on whether we allow a coupling between the two boundaries. We see an important point: a black hole is not just a "state", but a state with some evolution law. Only after specifying the evolution process can we can say that the black hole is "black" (it has a horizon). More specifically, if we start with the usual two boundary wormhole and we do not allow any information exchange between the two boundaries, then we have two black holes. But if we allow information exchange and also allow operators that couple the two boundaries, then we could have an eternal traversable wormhole, as in [17]. In both of these cases the "state" at $u_{l}=u_{r}=0$ is the same, or very similar. 


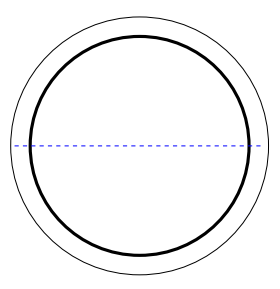

(a)

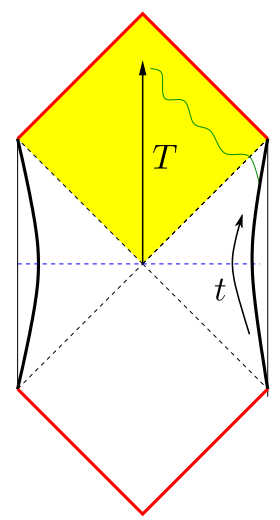

(b)

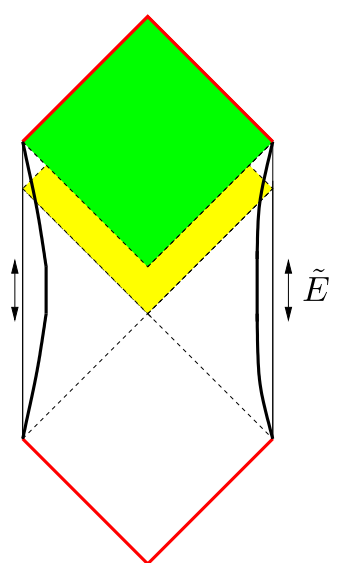

(c)

Figure 10. (a) The standard Euclidean $\mathrm{AdS}_{2}$ picture and its continuation to Lorentzian signature (b). This looks similar to the vacuum decays to AdS studied by Coleman and de Luccia [34]. They have shown that the addition of irrelvant operators at the domain wall, which is the boundary for us, leads to a divergence in the bulk at the red line. A bulk observer moving along the central black arrow gets to see a lot of the boundary in a very small proper time. This is the usual blue-shift near the inner horizon. In (c) we evolve for some time using the generator $\tilde{E}$. This allows us to explore some of the region that was behind the horizon, but the new horizon moves up. The yellow region was behind the horizon and now it is outside.

\subsection{The inner horizon or Cauchy horizon}

From the point of view of the matter in the bulk, the evolution is given by $E_{m}$ and it would seem at first sight that we could continue such evolution "forever". However, our present discussion does not allow us to move past the inner horizon or Cauchy horizon. The reason is the following. We assumed that the matter fields have standard boundary conditions at the $\mathrm{AdS}_{2}$ boundary. These are implied by the boundary conditions at the physical boundaries (curved black lines in figure 2). However, beyond the region where the physical boundaries extend, we have no guarantee that the matter boundary conditions are the same as when we had a physical boundary. For this reason we cannot extend the bulk evolution beyond the dotted red lines in figure (2). Note that this is also the boundary of the Wheeler de Witt patch when we move both the left and right times to the far future. Of course, it is an important problem to figure out what happens beyond that region!

It has been argued by Penrose that the inner horizon would be generically singular. (Though it has been demonstrated that classically the singularity is not too bad [35] and could be traversed, in some cases.) Here we can connect this expectation to the related discussion of vacuum decay into AdS that was studied by Coleman and de Luccia [34], see figure 10(a,b). In the thin wall approximation, the action of the bubble has a surface term and a boundary term, which reproduces JT gravity (2.12), [10, 11]. In our case, only the "true vacuum" part is present, not the false vacuum. Coleman and de Luccia have argued that in such situations there will be a singularity at the inner horizon. The reason is the following: imagine that we have a scalar field $\phi$ in the bulk and that there is some source 
for it on the boundary. Then, even if the field $\phi$ is massive, and thus corresponds to an irrelevant perturbation [36], it is expected to have a non-zero expectation value at the usual horizon. This is translation invariant in the FLRW patch (the yellow patch in figure 10(b)). Then the FLRW evolution will generically make it singular along the red line. (For a free bulk field one can avoid the singularity if the corresponding dimension $\Delta$ is an integer). In the case of the SYK model, we have other operators turned on when we are at finite $\beta \mathcal{J}$. For a near extremal $4 \mathrm{~d}$ charged black hole, the fact that the boundary conditions for the fields allow a leakage into the flat space region implies that we have some double trace operators turned on. This could imply that operators such as $\phi \phi$ would get divergent expectation values. This is a quantum effect. It is also suppressed in the scaling limit we took, but it seems important if $\beta \mathcal{J}$ is large and finite or the black hole throat has finite length.

Just to put in some formulas into this discussion we can consider a scalar field $\phi$ and imagine that there is some source on the boundary. We can then use the bulk-to-boundary propagator to compute

$$
\langle\phi\rangle \propto \int_{\mathcal{C}} d u \frac{1}{[-Y \cdot X(u)]^{\Delta}} \propto \int_{\mathcal{C}} d u \frac{1}{[\cos T+\sin T \sinh t(u)]^{\Delta}}
$$

where we took a point at the center of bulk with $Y^{a}=(\cos T, \sin T, 0)$ and a point at the boundary with $X \propto(1, \sinh t, \cosh t)$, see figure $10(\mathrm{~b})$. The inner horizon corresponds to $T=\pi$, and we see that there the $\sin T$ factor becomes zero and there could be a divergence from the integral over large real times. To analyze this properly we need to specify the contour of integration $\mathcal{C}$ which goes over the Keldysh contour appropriate for this problem. The regions that contribute are those where $Y$ and $X(u)$ are timelike separated, where the $i \epsilon$ prescription for the forwards and backwards parts Keldysh contour are different and do not cancel if $\Delta$ is not an integer. Formula (5.7) applies also for deformations by products of bulk field operators ("double trace"), where $\Delta$ in (5.7) is the total dimension of the operator.

Notice that this singularity can be moved by evolving the system for some time using the generator $\tilde{E}$, see figure 10 (c).

Note that the yellow region in figure 10(b) looks like a two dimensional FriedmanLemaitre-Robertson-Walker two dimensional cosmology. With the perturbations we discussed, it seems to develop a bulk singularity. A proper boundary understand of this region from the boundary theory would give us a toy model for a 2d FRLW cosmology. This is just the two dimensional version of a general connection between nearly conformal theories on $d S_{D-1}$ and negative cosmological constant FLRW cosmologies with $D-1$ hyperbolic slices [36].

\subsection{Moving operators into the bulk}

When we study gravitational systems with a boundary, one sometimes wants to express operators in the interior in terms of operators closer to the boundary. For example, if we had a bulk field $\phi(\tau, x)$ defined in the bulk of $\mathrm{AdS}_{2}$, we want to express the operator deep inside in terms of an operator closer to the boundary. One way to do it is via the HKLL construction [37] which involves solving the bulk wave equation and expressing the field 
at a point in the bulk as an integral of the field near the boundary over a range of times. Here we will provide an alternative construction.

We have constructed an operator $\tilde{P}$ which performs translations in the bulk, so we could use it to translate an operator deep in the interior to an operator closer to the boundary. Roughly we want

$$
\phi(\tau, x)=e^{i\left(x-x_{\mathrm{bdy}}\right) \tilde{P}} \phi\left(\tau, x_{\mathrm{bdy}}\right) e^{-i\left(x-x_{\mathrm{bdy}}\right) \tilde{P}}
$$

However, this expression is not good enough and we need to clarify some subtleties before writing a better expression.

First, recall that in the construction of $\tilde{P}$ we assumed that the actual UV boundaries where infinitely far away. Therefore the point $x_{\text {bdy }}$ should still be far away from the boundary, but it could sit at a relatively large value of the coordinates $x$ in (2.6a), a value that is large but fixed when we take the UV regulator to zero. In other words, we want $x_{\text {bdy }}$ to be larger than any value of $x$ of other operators in the bulk that we want to consider, but finite in the limit that we send the boundaries far away. A similar assumption goes into the standard HKLL [37] construction if one wants to use simple AdS wavefunctions.

A second issue is that the boundaries are dynamical objects and the coordinate points $x$ are not physical by themselves. When we construct the operator relatively close to the boundary we only determine its position relative to the boundary, we will call this $\ell_{\text {bdy }}$, or $x_{\mathrm{bdy}}=\ell_{\mathrm{bdy}}+x_{r}$, where $x_{r}$ is the position of the right boundary. Such an operator can be constructed in various ways, see e.g. [33]. For an operator with a large value of $\ell_{\text {bdy }}$ we expect that quantum fluctuations of the Schwarzian variables are small and the construction will be fairly accurate.

With all these caveats, we can now construct a better expression for a bulk operator at some distance $\ell$ from the UV boundary as

$$
\begin{aligned}
\Phi(\tau, \ell) & =e^{i\left(\ell-\ell_{\text {bdy }}\right) \tilde{P}} \Phi\left(\tau, \ell_{\text {bdy }}\right) e^{-i\left(\ell-\ell_{\text {bdy }}\right) \tilde{P}} \\
\Phi\left(\tau, \ell_{b d y}\right) & =\int d x_{l} d x_{r} \phi\left(\tau, \ell_{\text {bdy }}+x_{r}\right)\left|x_{l}, x_{r}\right\rangle\left\langle x_{l}, x_{r}\right|
\end{aligned}
$$

The first expression translates the operator from a point closer to the boundary to points deeper into the bulk. The second line expresses the operator close to the boundary in a gauge invariant fashion. This operator involves an operator $\phi$ acting on the matter Hilbert space. It also involves projection operators onto definite coordinate values for the boundary particles. Thus, it acts on the full Hilbert space of the theory (2.15). The momentum SL $(2)_{g}$ gauge generator acts by shifting all $x$ coordinates in (5.10) by a constant, which can be absorbed by a shift of integration variables. We have used a capital $\Phi$ to express the final dressed operators.

Finally, a more precise description of the bulk operator would also involve the time boundary variables and is

$$
\Phi\left(u_{l}, u_{r}, \ell\right)=\int d^{2} X_{l} d^{2} X_{l} \phi\left[Y^{a}\left(X_{l}, X_{r} ; \ell\right)\right]\left|X_{l}, X_{r}\right\rangle\left\langle X_{l}, X_{r}\right|
$$


where $Y^{a}$ is a point determined as follows. First we find the geodesic going between $X_{l}^{a}$ to $X_{r}^{a}$. Then we determine its midpoint. Then we move by a distance $\ell$ to the right along that geodesic to determine $Y^{a}$.

One can check that $\left[\Phi(\tau, \ell), \Phi\left(\tau, \ell^{\prime}\right)\right]=0$ for $\ell \neq \ell^{\prime}$. This should be compared to the HKLL construction where the construction has to be modified order by order to ensure this commutativity $[38,39]$. The construction discussed here includes the full gravitational dressing to all orders in the $G_{N}$ expansion. ${ }^{12}$ Furthermore, all matter self interactions have been taken into account. The prescription is somewhat similar to the prescription of "shooting a geodesic orthogonal to the boundary" [41, 42]. One issue is that, in AdS, all spacelike geodesics are orthogonal to the boundary. ${ }^{13}$ Here we choose a precise geodesic by selecting two boundary points, one on the left and one on the right.

Note that the whole discussion in this subsection is about the bulk theory, not the holographic boundary theory. Of course, it is convenient for holography to have the bulk operators written in terms of operators near the boundary.

\section{Connection to SYK and other systems}

\subsection{Generators in SYK}

Here we discuss these generators in the context of SYK. If we consider the system at temperature $\beta$ the effective coupling is $\beta J$. It is convenient to consider the limit

$$
\beta J \rightarrow \infty, \quad N \rightarrow \infty, \quad \frac{N}{\beta J}=\text { fixed }
$$

where the Schwarzian action becomes exact [14]. It is important to remark that, in this limit, we can consider quantum mechanical effects in the Schwarzian action. Also, since $S_{0} \propto N \rightarrow \infty$, other topologies do not contribute.

The constructions we discussed above for the charges can be discussed in this model. Whenever we got an expression involving $X_{l} . X_{r}$ we could replace it by a fermion operators via

$$
\frac{1}{\left(-2 X_{l} \cdot X_{r}\right)^{\Delta}} \propto \frac{i}{N} \sum_{j} \psi_{l}^{j} \psi_{r}^{j}
$$

Our expressions for the charges involved functions of $X_{l} . X_{r}$. We can then consider functions of these correlators, such as the logarithm or other powers. In the limit (6.1) these functions are well defined for the low energy states under consideration. Namely, one can be worried that the operator in the right hand side of (6.2) has zero or negative eigenvalues. However, in the large $N$ limit (6.1), we do not access such eigenvalues from low energy states. For such low energy states, and in the limit (6.1), the operator in the right hand side (6.2) is a positive operator. Therefore we can raise it to arbitrary powers (positive and negative) and we can also take its logarithm in order to construct the exact generators (3.6) (3.9).

\footnotetext{
${ }^{12}$ Due to topology changing corrections, of order $e^{-S_{0}}$, this prescription is not well defined nonperturbatively, see [40].

${ }^{13}$ One could still select a unique geodesic by choosing more than one point along the boundary, we discuss this in section (4.5). In our case, this can only work in the semiclassical limit.
} 
Now, one can say that in the infinite $N$ limit, (6.1), we have an infinite number of fermions anyway so it is not at all surprising that we can find some exact SL(2) algebra. What is interesting is that the quantum effects of the boundary mode are still finite in this limit, (6.1). In particular, the scrambling time for excitations of thermal energies is still finite, in this limit. And also the dynamics of the boundary mode is not conformal invariant. The non-trivial statement we are making is that, despite these facts, we still have exact $\mathrm{SL}(2)$ generators.

Most of our discussion used the language of nearly- $\mathrm{AdS}_{2}$ gravity. However, the SYK model displays the same structure, as reviewed in section (2.3). So everything we did in this paper also holds for the SYK model. Note that we made an important assumption when we discussed the JT gravity theory, right after (2.12). We said that the boundary was very far away. This scaling limit of the JT theory, where $\phi_{b} \rightarrow \infty$, is essentially the same as (6.1) in SYK. Unfortunately, the $G, \Sigma$ action does not give us a simple Hilbert space description, other than (2.15). In particular, one would like to understand how this emerges from the fermions. Or how the Hilbert spaces in (2.15) are embedded into the full Hilbert space of the fermions. The analysis of aspects of these symmetries directly in terms of the femions was undertaken in $[20,43]$. The results in this paper provide a "target" for such discussions.

Of course, an important question is how this structure is broken at finite $N$. We will not discuss that in this paper. However, we will now make the following simple observation. The action of the generator $\hat{E}_{c}$ (or $\hat{P}_{c}$ ) on a state created by a fermion was discussed near (4.12). Once we use the expression (4.29) (4.23) for the $\hat{E}_{c}$ generator, then the computation boils down to a fermion four point function computation of the general form

$$
\mathfrak{s} \frac{\left\langle\psi^{i}(\pi) \psi^{j}\left(u_{t}\right) \psi^{i}(0) \psi^{j}\left(u_{b}\right)\right\rangle}{\left\langle\psi^{i}(\pi) \psi^{i}(0)\right\rangle\left\langle\psi^{j}\left(u_{t}\right) \psi^{j}\left(u_{b}\right)\right\rangle}, \quad \mathfrak{s} \propto \frac{N}{\beta J}
$$

and there is no sum over $i$ or $j$. What we want is that this ratio of correlators is a certain particular order one function of the $\tilde{u}_{i}$ variables. Now, the general structure of the four point function is

$$
\frac{\left\langle\psi^{i}(\pi) \psi^{j}\left(u_{t}\right) \psi^{i}(0) \psi^{j}\left(u_{b}\right)\right\rangle}{\left\langle\psi^{i}(\pi) \psi^{i}(0)\right\rangle\left\langle\psi^{j}\left(u_{t}\right) \psi^{j}\left(u_{b}\right)\right\rangle}=1+\frac{\beta J}{N} \mathcal{F}_{\text {enhanced }}+\frac{1}{N} \mathcal{F}_{\text {finite }}
$$

where $\mathcal{F}_{\text {enhanced }}$ comes from the Schwarzian mode. The 1 is subtracted with the expectation value in (4.29). Due to the factor of $\mathfrak{s}$ in (6.3) we pick up only the part involving $\mathcal{F}_{\text {enhanced }}$ and the $\mathcal{F}_{\text {finite }}$ part drops out. In fact this last term contains the conformal invariant contribution that features an infinite sequence of composite operators, etc. Such terms depend on something which we can call "bulk interactions" [44]. Such terms drop out in the limit (6.1). If had not taken that limit, then we see that there are some specific $1 / \mathfrak{s}$ corrections that would change the action of $\hat{E}$ relative to that expected for an $\mathrm{SL}(2)$ generator. We will not discuss here whether this can be fixed up, or to what extent. But it is of course an interesting problem! 


\subsection{Relation to "size"}

It is worth noting that in SYK language the three generators in (4.23), (4.29), (4.31) can be expressed as

$$
\begin{aligned}
\hat{B} & =\frac{\beta}{2 \pi}\left(H_{r}-H_{l}\right) \\
\hat{E} & =\frac{\beta}{2 \pi}\left[H_{r}+H_{l}+i \mu \sum_{j} \psi_{l}^{j} \psi_{r}^{j}-\left\langle H_{r}+H_{l}+i \mu \sum_{j} \psi_{l}^{j} \psi_{r}^{j}\right\rangle_{\mathrm{TFD}}\right] \\
\hat{P} & =-i[\hat{B}, \hat{E}]=-i \frac{\beta^{2}}{4 \pi^{2}}\left\{i \mu \psi_{l}^{i}\left[H_{r}, \psi_{r}^{i}\right]-i \mu\left[H_{l}, \psi_{l}^{i}\right] \psi_{r}^{i}\right\} \\
& =-i \mu \frac{\beta^{2}}{4 \pi^{2}}\left(\psi_{l}^{i} \dot{\psi}_{r}^{i}-\dot{\psi}_{l}^{i} \psi_{r}^{i}\right) \\
\text { with } \quad \frac{\mu}{\mathcal{J}} & =\frac{4 \alpha_{S}}{\Delta c_{\Delta}}\left(\frac{\pi}{\beta \mathcal{J}}\right)^{2-2 \Delta}=\frac{\alpha_{s}}{\Delta}\left(\frac{2 \pi}{\beta \mathcal{J}}\right)^{2} \frac{1}{G\left(\frac{\beta}{2}\right)}
\end{aligned}
$$

where $G\left(\frac{\beta}{2}\right)=c_{\Delta}\left(\frac{\pi}{\beta \mathcal{J}}\right)^{2 \Delta}$.

On the other hand, in previous investigations, the concept of the "size" of an operator was of interest [18-21]. Roughly speaking, in SYK, the size of an operator counts the number of fermions that will be affected by applying this operator. One way to characterize this is to consider its commutator with all the fermion operators. ${ }^{14}$ For an operator normalized so that $2^{-\frac{N}{2}} \operatorname{tr} O^{\dagger} O=1$, the operator size is given by [21]:

$$
S_{\infty}(\mathcal{O})=\frac{1}{2} \sum_{i=1}^{N} 2^{-\frac{N}{2}} \operatorname{tr}\left(\left[\mathcal{O}, \psi^{i}\right]^{\dagger}\left[\mathcal{O}, \psi^{i}\right]\right) .
$$

For an operator $\psi\left(u_{r}\right)=\sum_{k} \sum_{i_{1}<\ldots<i_{k}} c_{i_{1} \ldots i_{k}}\left(2^{\frac{k}{2}} \psi_{i_{1}} \ldots \psi_{i_{k}}\right)$, this expression ${ }^{15}$ gives $S_{\infty}=$ $\sum_{k} \sum_{i_{1}<\ldots<i_{k}} k\left|\sqrt{2} c_{i_{1} \ldots i_{k}}\right|^{2}$. This can be written as the expectation value of some "size" operator defined as

$$
\begin{aligned}
\hat{S} & =\sum_{j} i \psi_{l}^{j} \psi_{r}^{j}+\frac{N}{2} \\
S_{\infty}(\mathcal{O}) & =\left\langle I\left|\mathcal{O}_{r}^{\dagger} \hat{S} \mathcal{O}_{r}\right| I\right\rangle,
\end{aligned}
$$

where $|I\rangle$ is the infinite temperature thermofield double state. ${ }^{16}$ Note that part of the global energy operator $\hat{E}$ in (6.6) appears here.

The above notion of size only depends on the operator. A generalization of this notion of size which depends on both the operator and the temperature $1 / \beta$ of the system is defined in [20], see also [19]:

$$
S_{\beta}(\mathcal{O})=\delta_{\beta}^{-1}\left(S_{\infty}\left(\mathcal{O} \rho^{\frac{1}{2}}\right)-S_{\infty}\left(\rho^{\frac{1}{2}}\right)\right) .
$$

\footnotetext{
${ }^{14}$ In the following expressions we assume the operator $\mathcal{O}$ is bosonic. When the operator we are interested in is fermionic, we can multiply it by a fermionic operator from some external system that anticommutes with all $\psi^{i}$ s.

${ }^{15}$ There is a factor of $\sqrt{2}$ due to the normalization $\left(\psi_{i}\right)^{2}=\frac{1}{2}$.

${ }^{16}|I\rangle$ satisfies $\left(\psi_{L}^{j}+i \psi_{R}^{j}\right)|I\rangle=0$. As a consequence $\langle I|\hat{S}| I\rangle=0$.
} 
Here $\rho$ is the thermal density matrix and $\delta_{\beta}$ is some normalization factor ${ }^{17}$ which fixes $S_{\beta}\left(\psi^{1}\right)=1$. This overall renormalization also implies $S_{\beta}\left(\psi^{1}(u)\right)$ saturates at the value $\frac{N}{2}$. In the limit that $\beta \rightarrow \infty$, we recover (6.9). We may also write this in terms of the size operator $\hat{S}$ :

$$
S_{\beta}(\mathcal{O})=\delta_{\beta}^{-1}\left(\frac{\left\langle T F D\left|\mathcal{O}_{r}^{\dagger} \hat{S} \mathcal{O}_{r}\right| T F D\right\rangle}{\left\langle T F D\left|\mathcal{O}_{r}^{\dagger} \mathcal{O}_{r}\right| T F D\right\rangle}-\langle T F D|\hat{S}| T F D\rangle\right)
$$

where the expectation value is taken on thermofield double at temperature $1 / \beta$.

Here we see that size is related to global energy. Let $\mathcal{U}_{r}$ be a unitary operator acting from the right side. Then, once the coefficients are adjusted and the factors of $H_{l}$ and $H_{r}$ are added as in (6.6), (6.5), (6.8), the size of $\mathcal{U}_{r}$ is

$$
S_{\beta}\left(\mathcal{U}_{r}\right)=\frac{\Delta}{2 \alpha_{s}} \frac{\beta \mathcal{J}}{2 \pi}\left\langle T F D\left|\mathcal{U}_{r}^{\dagger}(\hat{E}-\hat{B}) \mathcal{U}_{r}\right| T F D\right\rangle
$$

The fact that $\mathcal{U}_{r}$ is unitary is useful because it ensures that the term involving $H_{l}$ drops out from (6.14).

There are some interesting consequences of this expression. First, the $\hat{B}$ term is not important if one is only interested in the time dependence of size as we move a given operator insertion to earlier and earlier times (which was the focus of $[19,20]$ ), since in that case the energies $H_{r}$ and $H_{l}$ are conserved. Let $\mathcal{U}_{r}\left(u_{r}\right)=e^{i H_{r} u_{r}} \mathcal{U}_{r} e^{-i H_{r} u_{r}}$. It was found that its size grows as $\left|u_{r}\right|$ gets large. This growth is the growth of the corrections to the usual out of time ordered correlators if we expand out (6.13).

Using (6.14), we have

$$
\frac{\left.\partial S_{\beta}\left(\mathcal{U}\left(u_{r}\right)\right)\right)}{\partial u_{r}}=-i \frac{\Delta}{2 \alpha_{s}} \frac{\beta \mathcal{J}}{2 \pi}\left\langle\mathcal{U}_{r}\left(u_{r}\right)^{\dagger}\left[\frac{2 \pi}{\beta} \hat{B}, \hat{E}\right] \mathcal{U}_{r}\left(u_{r}\right)\right\rangle=\frac{\Delta}{2 \alpha_{s}} \mathcal{J}\left\langle\mathcal{U}_{r}\left(u_{r}\right)^{\dagger} \hat{P} \mathcal{U}_{r}\left(u_{r}\right)\right\rangle
$$

We see that the momentum is related to the time derivative of size. This is also explored in [45].

The size of such an operator at large $q$ was computed in SYK in [20]. To make such a perturbation at $u_{r}=0$, we insert an operator of dimension $\Delta$ to the Euclidean circle at $\varphi_{b}=\frac{2 \pi}{\beta}\left(-\frac{1}{2 \mathcal{J}}+i u_{r}\right), \varphi_{t}=\frac{2 \pi}{\beta}\left(\frac{1}{2 \mathcal{J}}+i u_{r}\right)$. We give a small real part to $\varphi$ to smear the operator by the amount $\sim \frac{1}{\mathcal{J}} \cdot{ }^{18}$ Using (4.18) (and similar formulas for the other two generators obtained through (4.12)) we can get the charges of such an excitation.

$$
\begin{aligned}
(\hat{B}, \hat{P}, \hat{E}) & =\left(\Delta \frac{1}{\tan \frac{\varphi_{t}-\varphi_{b}}{2}},-i \Delta \frac{\sin \frac{\varphi_{t}+\varphi_{b}}{2}}{\sin \frac{\varphi_{t}-\varphi_{b}}{2}}, \Delta \frac{\cos \frac{\varphi_{t}+\varphi_{b}}{2}}{\sin \frac{\varphi_{t}-\varphi_{b}}{2}}\right) \\
& \sim \Delta \frac{2 \beta \mathcal{J}}{2 \pi}\left(1, \sinh \left(\frac{2 \pi}{\beta} u_{r}\right), \cosh \left(\frac{2 \pi}{\beta} u_{r}\right)\right)
\end{aligned}
$$

\footnotetext{
${ }^{17} \delta_{\beta}=2 G\left(\frac{\beta}{2}\right)=2 c_{\Delta}\left(\frac{\pi}{\beta \mathcal{J}}\right){ }^{2 \Delta}$, see $[20]$.

${ }^{18}$ The amount of smearing needed to go from UV to the conformal regime is $\sim \frac{1}{\mathcal{J}}$. We determined the $\frac{1}{2}$ factor in the real part of $\varphi_{t}$ and $\varphi_{b}$, by matching the energy to the energy of the exact solution at large $q[9]$. The energy can be computed by taking time derivative of the two-point function.
} 
where we assumed $\left|\varphi_{t}-\varphi_{b}\right| \ll 1$. Using (6.14) we find its size

$$
S_{\beta}\left(\psi\left(u_{r}\right)\right)=\frac{\Delta}{2 \alpha_{s}} \frac{\beta \mathcal{J}}{2 \pi}(\hat{E}-\hat{B})=2 \frac{\Delta^{2}}{\alpha_{s}}\left(\frac{\beta \mathcal{J}}{2 \pi}\right)^{2} \sinh ^{2}\left(\frac{\pi}{\beta} u_{r}\right)
$$

This agrees with the explicit fermion counting calculation in [20] at large $q$.

In the above example, the excitation has boost energy $2 \Delta \mathcal{J}$. It starts from near the boundary and falls toward the horizon. A lower energy excitation can be made by creating a particle at rest at a finite proper distance to the horizon $\rho_{m}$. We denote an operator which creates such an excitation by $\tilde{\psi}\left(\rho_{m}\right)$. We can compute the size of such an operator using (6.14). To create such an excitation, one can consider insertions at some finite angle $\varphi_{t}=-\varphi_{b}$ on the Euclidean circle, see figure 6 . Its distance to the horizon is $\rho_{m}=-\log \left(\tan \left(\frac{\varphi_{t}}{2}\right)\right)$. We write its charges (4.19) in terms of $\rho_{m}$ :

$$
(\hat{B}, \hat{P}, \hat{E})=\left(\Delta \sinh \rho_{m}, 0, \Delta \cosh \rho_{m}\right)
$$

The size of this single-fermion perturbation ${ }^{19}$ is

$$
S_{\beta}\left(\rho_{m}\right)=\frac{\Delta^{2}}{2 \alpha_{s}} \frac{\beta \mathcal{J}}{2 \pi} e^{-\rho_{m}} .
$$

When the excitation is near the boundary, $\rho_{m} \sim \log (\beta \mathcal{J})$ and the size is of order 1 . As we move the excitation deeper and deeper into the throat its size increases exponentially and reaches $\sim \beta \mathcal{J}$ before it enters the near-Rindler region.

In fact, one can directly see this exponential growth from the commutation relation $[P, E-B]=i(E-B) .{ }^{20}$ We consider the operators $\tilde{\psi}(\rho)=e^{-i \hat{P}\left(\rho-\rho_{b}\right)} \psi e^{i \hat{P}\left(\rho-\rho_{b}\right)}$.

$$
\begin{aligned}
\frac{d}{d \rho} S_{\beta}(\tilde{\psi}(\rho)) & =\frac{\Delta^{2}}{2 \alpha_{s}} \frac{\beta \mathcal{J}}{2 \pi} 2\langle T F D|\tilde{\psi}(\rho)[i \hat{P}, \hat{E}-\hat{B}] \tilde{\psi}(\rho)| T F D\rangle \\
& =-S_{\beta}(\tilde{\psi}(\rho))
\end{aligned}
$$

Part of the message of this paper is that the symmetry structure in (2.14) (2.15) is giving us the correct "target" generators that should be reproduced by the microscopic analysis. Moreover, to the extent that we have derived the Schwarzian theory from SYK, we have also derived the existence of these generators from the SYK model.

In [45] Susskind discusses the following relation between the momentum of an infalling particle and the complexity of a precursor preparing this perturbation

$$
P \sim \frac{d}{d u_{r}} \mathcal{C} .
$$

The complexity-volume conjecture relates the length of the wormhole with the state complexity [46]. Using this we see that (6.20) follows from (3.7).

\footnotetext{
${ }^{19}$ In this formula, as well as in (6.17), one factor of $\Delta$ originates from the dimension of the operators used to couple the two sides in our expressions for the global energy, whereas the other factor of $\Delta$ is the dimension of the operator inserted. So the size of a more general operator $O$ of dimension $\Delta_{O}$ would be $S_{\beta}(O) \propto \Delta \Delta_{O}$.

${ }^{20}$ Here we only consider small excitations on the throat so to a good approximation we can assume the SL(2) algebra holds. See section 4.3.
} 


\subsection{Analogy to the Rindler (and AdS-Rindler) decomposition of a higher di- mensional field theory}

In section 4.3 we discussed the construction of approximate symmetry generators. One generator, $\hat{B}$ in (4.23), is the sum of an operator defined purely on the left and one purely on the right. This generator acts within the entanglement wedges of each of the two sides. On the other hand, the operator (4.29) is the sum of pieces that act on each side separately plus a term containing a product of left and right operators. This might seem exotic for a symmetry generator.

Here, we will point out that this type of structure is actually what we get in an ordinary quantum field theory when we use Rindler coordinates. The analogy is most clear if think of a quantum field theory on a spatial sphere. We then divide the sphere in two halves. We then automatically have a generator like $\hat{B}$. This is just the full modular Hamiltonian of the hemisphere (or a solid ball). The full state of the quantum field theory is exactly the thermofield double for the modular energy. In the bulk, each entanglement wedge looks like the outside of a hyperbolic black hole [47].

Now we can consider a generator such as the global time translation Killing vector of the full system. This generator is exactly related to an integral of the form

$$
E=\int_{\text {Sphere }} T_{00}=E_{l}+E_{r}+\sum_{i} \phi_{l}^{i} \phi_{r}^{i}, \quad E_{l}=\int_{l-\text { Hemisphere }} T_{00}
$$

where $E_{r}$ is the integral over the right hemisphere and $\phi_{l} \phi_{r}$ is related to the stress tensor on exactly the boundary between the two hemispheres. In order to make it more manifest, we could regularize the theory using a lattice so that there is no lattice point precisely at the boundary. However, there is a term in the full global Hamiltonian which acts on the sites that are to the left and the right of the boundary and these terms have the structure of an operator on the left and one on the right side. The sum over $i$ is over all fields of the boundary theory. ${ }^{21}$

So we see that the structure of $\hat{E}$ in (4.29) is very reminiscent of what we have in an ordinary QFT when we split it into two parts and we consider the generator $E$. Instead of $H_{r}+H_{l}$ (which here would be $B_{l}+B_{r}$ ), we have two other operators that are defined purely on the left and the right in (6.21).

The fact that there are terms that act on both the left and the right is crucial to generate the appropriate isometry which can be used to transfer information between the left and right system. In fact, this is the teleportation operator introduced in [16]. Note also that in (6.21) we naturally have many operators coupling the left and the right because we have many fields in a CFT that is dual to a weakly coupled gravity system. In fact, we can view a particle moving in the gravitational bulk from one side to the other as a particular example of the traversable wormhole discussion in [16], but applied to the hyperbolic black holes.

However, one important difference between the higher dimensional case and the two dimensional gravity theory is that in higher dimensions we can define an operator $E$, as

\footnotetext{
${ }^{21}$ If we had a gauge theory we can do the splitting by introducing extra boundary charges (in an entangled state) and we have the same structure [48].
} 


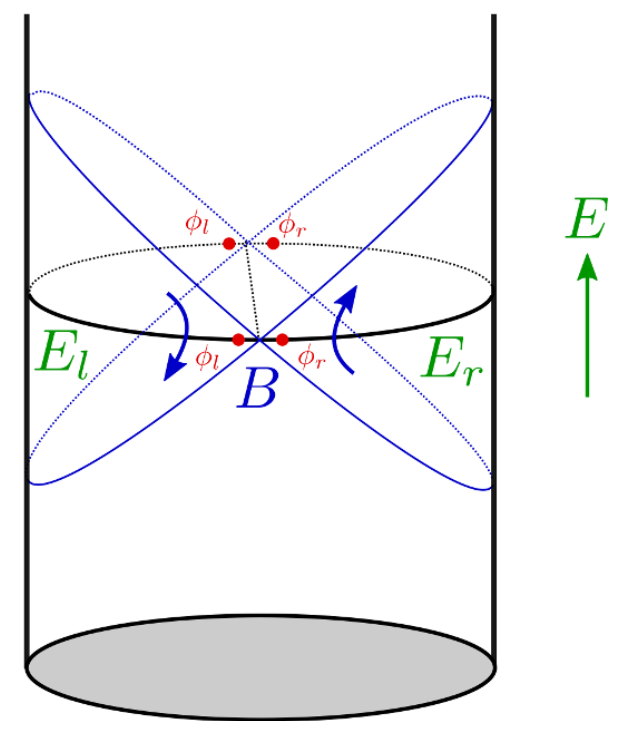

Figure 11. We show a two dimensional boundary theory on a cylinder. We divide the cylinder in two parts, a left and right part. The full modular Hamiltonian corresponds the boost like generator $B$, which also acts like a boost in the bulk near horizon region. The global time translation generator $E$ is the sum of three parts. $E_{l}$ and $E_{r}$ are completely contained in each region. The third term involves a product of operators in the two regions, depicted here in red.

in (6.21), that is part of an exact $\mathrm{SO}(2, D-1)$ conformal algebra. In our case, we only had an approximate expression.

Notice that in the CFT problem, even if all we cared about was the theory on $R \times H_{d-1}$, or the theory on the hemisphere, then when we consider the thermofield double of that system we generate an entangled state which, in the bulk, could be extended beyond the union of the two entanglement wedges. This region can be easily explored by evolving the system with the energy generator $E$ (6.21).

\section{Discussion}

\subsection{Measuring distance}

In the context of a theory of gravity plus matter the distance between the two boundaries look like a reasonable observable (at least if we ignore the effects of topology change, see [29]).

On the other hand, from the point of view of the holographic boundary theory (the quantum mechanical theory with a finite number of degrees of freedom), this distance is not an obvious operator. We will not attempt to give it a precise meaning in the boundary theory here. It was proposed in $[46,49,50]$ that this distance is related to "complexity".

It will suffice to note that we can define it by considering correlation functions of operators. Namely, one can consider correlation functions of operators across the two boundaries, such as

$$
\left\langle O_{l} O_{r}\right\rangle=\frac{1}{\left(-X_{l} \cdot X_{r}\right)^{\Delta}}
$$


This is a correlation function on the vacuum and by measuring it, we can indirectly infer the distance [51]. However, if we define the distance in terms of this operator, then we could change it by changing the state of this field. For example, we can act with an operator that creates a highly correlated state between left and right values of the field. We can avoid this by imagining that we have many fields, then we can take the average of the correlators of many fields. In that case, changing just one field is not enough to change the distance. But it could be that, if we changed all $N$ of the fields, then we could generate a new geometry. For this to work, the number $N$ has to be comparable to $S_{0}$, the extremal entropy. ${ }^{22}$ In the SYK we indeed have $N$ fields and an $S_{0} \propto N$, so that this method makes sense. This simple method of measuring distances works in the limit (6.1). In appendix G, we explore how to measure distance in the opposite regime: a bulk theory with only one free scalar field.

As a qualitative side comment we could say that by increasing the correlations for just one field, we are introducing a small microscopic wormhole, which could become macroscopic when we correlate all $N$ fields.

A second problem with the definition of distance in terms of correlators is that if we have bulk matter, we can have interactions with matter. If we have such interactions, the correlators could change in the presence of bulk matter and our definition of distance will change. In the particular case of SYK model, these self interactions go like $1 / N$, and are suppressed in the limit (6.1). Therefore, in this strict limit this definition makes sense.

In more general $\mathrm{NAdS}_{2}$ theories, it is more challenging problem to define this distance, and we will not attempt to do it here. By more general theories we mean, for example, ones with a small number of matter fields where these fields are self interacting.

A potentially promising framework for understanding the distance between the two boundaries is the following. ${ }^{23}$ In many body physics, there are some properties that are not defined for single particles but emerge when we have many particles. An example is the phase of a superconductor (by a "superconductor" here we just mean a system of interacting fermions with a $\mathrm{U}(1)$ global symmetry that is spontaneously broken). This phase is good classical variable in the large $N$ limit, but it is not well defined for a small number of fermions. In our case, we have a rather similar variable which is the relative time shift between the two sides of the thermofield double. This relative time shift behaves as a classical variable and it is indeed one of the classical variables of an empty wormhole $[28,29]$. It can be measured by considering correlation functions, in the same way that the phase of a superconductor can be measured looking at fermion two point functions. One subtle point for our discussion is that in our case this is both spontaneously and explicitly broken. In other words, if we evolve by $H_{l}+H_{r}$ we increase the value of this time shift. This is analogous to adding a term to the superconductor Hamiltonian that is proportional to the $\mathrm{U}(1)$ charge; the phase would then move linearly with time. We think that we can call this a "time superfluid" in the sense that the overall time translation symmetry is broken by the wormhole or thermofield double state. In fact, we can think about any state that has a classical time dependence as a "time superfluid" in the sense that the time translation

\footnotetext{
${ }^{22}$ Ideas for measuring distance were mentioned in [51].

${ }^{23}$ See a related discussion in [52].
} 
symmetry is broken. The wormhole seems special because this time translation is also related to other symmetries of the problem, the approximate SL(2) symmetries of the quantum theory. So another very close to flat direction is the temperature. Namely, states with different temperatures are also related. They are connected by acting with an overall dilation the system. This affects the wormhole by making it longer. In fact, in the wormhole both the time and space directions are related, so the state should be more fittingly called a "space-time superfluid". We hope to expand on these remarks in a future publication.

\subsection{Conclusions and open questions}

In this paper we have studied the symmetries near the horizon of a black hole. The main reason to study them is that these allow us to move into the region behind the horizon, in the sense discussed in section 5.2. So a thorough understanding of these symmetries is crucial for understanding how the interior region emerges in the full quantum theory. We have considered near extremal black holes because in this case we have a connection to the SYK model. We considered a scaling limit where the temperature becomes very small and the extremal entropy very large, but the near extremal entropy remains fixed. This physically means that we are keeping the scrambling time fixed in units of the temperature and quantum gravity effects are important, but calculable. But topology changing effects are negligible. We have defined three SL(2) generators (3.1) (3.2). They act on both the matter degrees of freedom and the boundary graviton degrees of freedom. If we have a state with fixed values of the boundary positions, the generators do not move the boundaries but move the matter relative to the boundaries as in figure 2. These generators act on physical states of the theory grouping them into SL(2) representations. In particular, they imply that the number of states for the wormhole is infinite. This is not a contradiction because we are working in a limit where the extremal entropy is infinite. The non-trivial aspect is that we are keeping the scrambling time finite. So we are making the non-trivial statement that the generators are well defined even after the scrambling time. These generators do not commute with the Hamiltonian, so we cannot call them "symmetries", though from the point of view of a bulk observer made out of matter they act pretty much like symmetries. The total Hamiltonian changes the generators because it changes the boundary positions. Fortunately the evolution of the boundary positions is a solvable quantum problem so that, in principle, we can undo it. This allows us to define time independent (conserved) charges as in (3.10).

We have also expressed the generators in terms of the distance between the left and right boundaries and their time derivatives. In this way we obtain expressions that depend purely on the boundary and its dynamics. (Of course, this is related to matter via the constraints.) These formulas also allow us to express the generators in terms of correlation functions of operators, after making some extra assumptions. These assumptions are necessary to relate correlation functions to distances between the boundaries, even in the presence of matter. We have assumed that we have a number of fields scaling like $N$ and that their interactions with the matter we are probing goes as $1 / N$. These conditions are met in the SYK model. 
In the semiclassical limit, where $\Delta S=\mathfrak{s} \gg 1$, we can further simplify the expressions for the generators and write them in terms of products of operators on the two boundaries. These are similar to the operators that appear in the traversable wormhole discussion [16]. This is not a coincidence, it is because these operators generate bulk matter displacements across the horizon. These displacements also play a role in the growth of chaos or in out of time order correlators. We have also shown that these operators approximately act like $\mathrm{SL}(2)$ transformations on the boundary time $u$, so that they can be viewed as conformal ${ }^{24}$ transformations of the boundary theory. This also allows us to define an operator-state mapping. It relates operators inserted during Euclidean evolution by $\beta / 2$ to states on the wormhole. These approximate symmetry generators depend explicitly on the temperature of the background wormhole we are expanding around.

We should emphasize that our discussion is valid both for a nearly- $\mathrm{AdS}_{2}$ gravity theory with a large number of fields or the SYK model. In both cases, we can repeat all the steps of this construction. And the discussion is valid in the large $N$ limit with finite $N /(\beta J)$. This means that scrambling effects are included, but not effects due to the finite distance to the boundary (finite $\beta J$ ), or topology changing finite $N$ effects. As an example, we have computed the growth of the charges before and beyond the scrambling time in section 4.2.2 and found that beyond the scrambling time the momentum or energy of an excitation does not continue to grow. This saturation is related to the fact that out of time order correlators decay to zero. In fact, when we evaluate the charges on a state that is created by inserting operators during euclidean evolution, we are computing the same type of correlation functions that appear in out of time order correlators. These out of time order correlators have some pieces that display an exponential growth related to chaos. It is also possible to define the approximate generators in such a way that they depend only on these growing pieces. Of course, this is related to the well known fact that such pieces are related to shock wave scattering, which in this two dimensional context, generate simple bulk displacements [30,31]. Here we are inverting the logic and saying that we can use these growing correlators to define symmetry generators. It is tempting to say that in any system with maximal chaos we can define translation generators that, together with the boost generator obey an approximately Poincare algebra near the horizon. For near extremal black holes they obey an $\mathrm{SL}(2)$ algebra, which is a bit more constraining. The difference is just whether $[E, P]$ is zero or $[E, P] \propto B$. Even in the $\mathrm{SL}(2)$ case, if we consider excitations very close to the horizon, at distances smaller than the radius of $\mathrm{AdS}_{2}$, we find that $E$ and $P$ become relatively large, so that after a rescaling we obtain a Poincare looking algebra. It would be nice to understand the conditions under which this structure emerges in a general maximally chaotic large $N$ system.

The approximate generators are related to the "size" operator that has been studied recently [18-21]. This connection to a symmetry algebra explains many of its features and helps to clarify its relation to bulk dynamics in $\mathrm{AdS}_{2}$. A particularly simple relation is that the time derivative of size is the same as the bulk momentum. This is explored further in [45]. In [20] a direct SYK analysis at large $q$ and large $N$ showed how size evolved up

\footnotetext{
${ }^{24}$ We are talking about three generators, and not the infinite dimensional group of reparametrizations.
} 
to the scrambling time. Presumably, similar methods could explain why it saturates after the scrambling time. In other words, one would like to have a better microscopic picture for these generators in the SYK model, one which does not go through the usual route of the $G, \Sigma$ action but rather constructs them more manifestly in terms of the UV operators of the model, as in [20].

There are some straightforward looking generalizations of this discussion, for example one could consider a supersymmetric system and a super-Schwarzian.

It would be interesting to understand how this story is modified when the length of the throat is not infinite (or $\beta J$ is finite) and eventually covering the case of a generic finite temperature black hole. We know that the chaos correlators are also given in terms of simple displacements in this case too. So we expect that they should also be useful for constructing the local $2 \mathrm{~d}$ Poincare symmetry near any horizon (the two dimensions are time and the radial direction). We have also recalled the general expectation that the inner horizon would have some kind of singularity. It would be interesting to understand it better, and it is likely that these finite throat length corrections are relevant.

\section{Acknowledgments}

We thank the anonymous referee, D. Harlow, L. Iliesiu, D. Jafferis, A. Lewkowycz, X.L. Qi, S. Shenker, D. Stanford, A. Streicher, L. Susskind, Z. Yang, and W. Zhao for discussions.

H.L. is supported by an NDSEG fellowship. J.M. is supported in part by U.S. Department of Energy grant de-sc0009988 and by the Simons Foundation grant 385600. Y.Z. is supported by the Simons foundation through the It from Qubit collaboration. We benefited from the workshop "Chaos and Order: From strongly correlated systems to black holes" at KITP, supported in part by the National Science Foundation under Grant No. NSF PHY-1748958 to the KITP.

\section{A $\quad \mathrm{SO}(3)$ analogy}

Consider a non-relativistic particle in Euclidean space. We have the position operators and the angular momenta:

$$
\left[J_{i}, J_{j}\right]=i \varepsilon_{i j k} J_{k}, \quad\left[J_{i}, X_{j}\right]=i \varepsilon_{i j k} X_{k}, \quad\left[X_{i}, X_{j}\right]=0, \quad i, j, k=1,2,3 .
$$

This is the Euclidean algebra $E(3)$. (Note that what is normally the momentum generator, $P^{i}$, is here the position operator). This algebra has two Casimirs,

$$
X^{2}=r^{2}, \quad J . X=\lambda .
$$

Our toy model consists of two non-relativistic particles, which we dub "left" and "right," each constrained to live on the surface of the sphere $r^{2}=1$. We assume for simplicity that our non-relativistic particles do not carry intrinsic spin, so $\lambda=0$. In addition, there is some "matter" which can carry angular momentum. This matter could for instance be some spinning particle, which lives at the center of the sphere. (Later we will see that the 
matter has to have integer spin.) Finally, we demand that the overall system has vanishing angular momentum $J_{l}+J_{r}+J_{m}=0$; e.g., the overall $\mathrm{SO}(3)$ symmetry is gauged. Thus the Hilbert space of our system may be denoted

$$
\mathcal{H}=\left(\mathcal{H}_{l} \otimes \mathcal{H}_{r} \otimes \mathcal{H}_{m}\right) / \mathrm{SO}(3) .
$$

This also means that all physical operators $O$ must commute with the total angular momentum, $\left[J_{l}^{a}+J_{r}^{a}+J_{m}^{a}, O\right]=0$.

\section{A.1 Exact $\mathrm{SO}(3)$ algebra from two copies}

Armed with this Hilbert space, we may consider the physical operators

$$
X_{l} . J_{r}, \quad X_{r} . J_{l}, \quad\left(X_{l} \times X_{r}\right) .\left(J_{l}+J_{r}\right) .
$$

Note that we can $\lambda=0$ and the gauge constraint to rewrite these as

$$
G^{1}=-X_{l} . J_{m}, \quad G^{2} \sim-X_{r} . J_{m}, \quad G^{3} \sim-\left(X_{l} \times X_{r}\right) . J_{m} .
$$

This suggests that the 3 operators form an $\mathrm{SO}(3)$ algebra. (More precisely, we should replace $X_{r}$ with the Gram-Schmidt linear combination of $X_{l}$ and $X_{r}$ that is orthonormal to $X_{l}$.) We can check this by computing their commutators directly in the 2-body Hilbert space without the gauge constraint. To do so, it is worth introducing a little bit of notation. Let $V$ and $W$ transform be vector operators under $\mathrm{SO}(3)$ such that

$$
\begin{array}{r}
{\left[V_{i}, V_{j}\right]=\left[W_{i}, W_{j}\right]=\left[V_{i}, W_{j}\right]=0} \\
V . V=W . W=1, \\
V . W=0 .
\end{array}
$$

Then defining $e^{1}=V, e^{2}=W, e^{3}=V \times W$, we may write $G^{A}=-e_{i}^{A} J_{i}$ where $J=J_{l}+J_{r}$. Here the capital indices are physical, and the lower indices are the gauge index.

$$
\begin{aligned}
{\left[G^{A}, G^{B}\right] } & =\left[e_{i}^{A} J_{i}, e_{j}^{B} J_{j}\right] \\
& =i \epsilon_{i j k}\left(e_{i}^{A} e_{k}^{B} J_{i}+e_{j}^{B} e_{i}^{A} J_{k}+e_{j}^{B} e_{k}^{A} J_{i}\right) \\
& =-i\left(e^{A} \times e^{B}\right) . J \\
& =i \epsilon^{A B C} G_{C},
\end{aligned}
$$

where in the last line, we used $e^{A} \times e^{B}=\epsilon^{A B C} e_{C}$. Note that in order to construct this algebra, it was crucial that we had other vector operators that were not just the angular momentum operators. The left and right Hilbert spaces were representations of the Euclidean algebra, and not just the $\mathrm{SO}(3)$ algebra, so we are necessarily considering an infinite dimensional Hilbert space.

It is also interesting to construct the Casimir operator

$$
C=G^{A} G^{A}=e_{i}^{A} J_{i} e_{k}^{A} J_{k}=\delta_{i k} J_{i} J_{k}=J^{2} .
$$

So we see that the Casimir of the gauge charges is equal to the Casimir of these physical charges. 
Note that evolving with one of the $G^{A}$ leads the $X_{l}$ and $X_{r}$ vectors to precess about some axis. Alternatively, if we replace $J_{l}+J_{r}$ with $-J_{m}$, we can view the positions of the particles as fixed under time evolution, but the matter rotates. The invariant statement is that the generators move the left and right particles with respect to the matter.

\section{A.2 Action on states/operators}

We would now like to use the physical algebra to organize states and operators in this theory. Let us imagine that the matter sector contains a vector operator $W^{a}$ which is not $J^{a}$. For example, if the matter is actually another particle, $W^{a}$ could be its position vector $Y^{a}$. We may form the gauge invariant operator $W^{A}=W^{a} e_{a}^{A}$. Then $\left[W^{A}, G^{B}\right]=i \epsilon^{A B C} W_{C}$. Furthermore, we can use $W^{A}$ to construct tensor operators. So we can generate operators which transform in any integer spin representation of the physical $\mathrm{SO}(3)$.

Now consider a state $|0\rangle$ that is in a singlet under the gauge $\mathrm{SO}(3)$ and the physical $\mathrm{SO}(3)$

$$
|0\rangle=\sum_{m, j} \psi(j)(-1)^{m}|j, m\rangle_{l} \otimes|j,-m\rangle_{r} \otimes|0,0\rangle_{\text {matter }} .
$$

Here $\psi(j)$ can be any normalizable function. For example, the thermofield double corresponds to $\psi \sim e^{-\beta j^{2} / 4}$. We may generate states which transform under integer spin by repeatedly applying $W^{A}$, e.g., $W^{A} W^{B} W^{C}|0\rangle$. We can then organize these into irreps $|j, m\rangle$ of the physical algebra.

One interesting question is: what matter states are allowed in this gauge theory? Since there are no half-integer states in the two-particle Hilbert space, we conclude that the matter is restricted to be integer spin. For example, the matter cannot be a spin $1 / 2$ qubit.

\section{B Canonical quantization of Schwarzian theory}

The purpose of this section is to show that the most naive quantization of the Schwarzian theory explicitly realizes the commutation relations needed in our construction of the charges. We will quantize using global time coordinates $T_{r}(u)$, although one could also use Rindler coordinates related by $\tan \left(T_{r} / 2\right)=\tanh \left(t_{r} / 2\right)$.

Friendly warning. When using global time, we adopt different embedding space conventions from the rest of the paper. Defining $\mathcal{X}^{0}=X^{1}$ and $\mathcal{X}^{ \pm}=X^{-1} \pm i X^{0}$, we may write

$$
\begin{aligned}
X_{r}=\left(\mathcal{X}_{r}^{0}, \mathcal{X}_{r}^{+}, \mathcal{X}_{r}^{-}\right) & =\left(\frac{1}{T_{r}^{\prime}},-\frac{e^{i T_{r}}}{T_{r}^{\prime}},-\frac{e^{-i T_{r}}}{T_{r}^{\prime}}\right), \\
X_{l} & =\left(-\frac{1}{T_{l}^{\prime}},-\frac{e^{i T_{l}}}{T_{l}^{\prime}},-\frac{e^{-i T_{l}}}{T_{l}^{\prime}}\right) .
\end{aligned}
$$

The metric in these coordinates is $X . Y=\mathcal{X}^{0} \mathcal{Y}^{0}-\frac{1}{2}\left(\mathcal{X}^{+} \mathcal{Y}^{-}+\mathcal{X}^{-} \mathcal{Y}^{+}\right)$. The advantage of this convention is that the components of $X$ are simple when written in terms of global time, and furthermore $(2.9)$ becomes $\left[\mathcal{Q}_{m}, \mathcal{Q}_{n}\right]=(m-n) \mathcal{Q}_{m+n}$, where $m, n$ take values from $\{0,+,-\}$. This convention is perhaps more familiar from $d \geq 2 \mathrm{CFT}$, where $L_{0}$ generates dilation, or global time translation along the cylinder. 
Let's proceed with the canonical quantization. We start with the Lagrangian on one side, setting $T=T_{r}\left(u_{r}\right)$ :

$$
L=-\{\tan T / 2, u\}=\frac{1}{2} \frac{T^{\prime 2}}{T^{2}}-\frac{1}{2} T^{2}-\left(\frac{T^{\prime \prime}}{T^{\prime}}\right)^{\prime} .
$$

Dropping the overall derivative, we search for a 4-dimensional phase space with 2 canonical coordinates $T, T^{\prime}$. The canonical momentum are $p_{i}=\sum_{k=i}^{2}\left(-\partial_{u}\right)^{k-i} \partial L / \partial T^{(k)}$ :

$$
p_{1}=-T^{\prime}+\frac{T^{\prime \prime 2}}{T^{\prime 3}}-\frac{T^{\prime \prime \prime}}{T^{\prime 2}}, \quad p_{2}=\frac{T^{\prime \prime}}{T^{\prime 2}}
$$

Note that $p_{1}$ is just the global $T$ gauge charge $p_{1}=\mathcal{Q}^{0}$. Then the Hamiltonian is

$$
H=\sum p \dot{q}-L=-\frac{1}{2} T^{2}+\frac{3 T^{\prime \prime 2}}{2 T^{\prime 2}}-\frac{T^{\prime \prime \prime}}{T^{\prime}}=-\{\tan T / 2, u\}
$$

We then impose canonical commutation relations

$$
\left[T, T^{\prime}\right]=0, \quad\left[T, p_{1}\right]=i, \quad\left[T^{\prime}, p_{2}\right]=i .
$$

We would like to check that the gauge charges satisfy an $\mathrm{SL}(2)$ algebra. The other two charges are

$$
\mathcal{Q}^{ \pm}=e^{ \pm i T}\left( \pm i \frac{T^{\prime \prime}}{T^{\prime}}+\frac{T^{\prime \prime 2}}{T^{\prime 3}}-\frac{T^{\prime \prime \prime}}{T^{\prime 2}}\right)
$$

Now to compute these commutators, we first rewrite these charges in terms of coordinates and momenta by eliminating $T^{\prime \prime \prime}, T^{\prime \prime}$ in favor of $p_{1}, p_{2}$.

$$
\mathcal{Q}^{ \pm}=e^{ \pm i T}\left(T^{\prime}\left(1 \pm i p_{2}\right)+p_{1}\right), \quad \text { and } \quad \mathcal{Q}^{0}=p_{1} .
$$

In writing these expressions, we have chosen an operator ordering where all the momenta are to the right of the coordinates. Note that these charges generate the expected infinitesimal transformations on the times and satisfy the $\mathrm{SL}(2)_{g}$ algebra:

$$
\begin{aligned}
i\left[\mathcal{Q}^{0}, T\right] & =1, & i\left[\mathcal{Q}^{0}, T^{\prime}\right] & =0, \\
i\left[\mathcal{Q}^{ \pm}, T\right] & =e^{ \pm i T}, & i\left[\mathcal{Q}^{ \pm}, T^{\prime}\right] & = \pm i e^{ \pm i T} T^{\prime} \\
{\left[\mathcal{Q}^{0}, \mathcal{Q}^{ \pm}\right] } & = \pm \mathcal{Q}^{ \pm}, & {\left[\mathcal{Q}^{+}, \mathcal{Q}^{-}\right] } & =-2 \mathcal{Q}^{0}
\end{aligned}
$$

With these gauge charges, $H$ is simply the Casimir

$$
H=-\frac{1}{2} Q \cdot Q
$$

One can check that, e.g., the equation of motion correctly links the two coordinates $i[H, T]=T^{\prime}$.

Now consider the embedding space vectors in (B.1). We see that the $X$ 's are only a function of the coordinates and not the momenta. So we immediately conclude that all components of $X$ commute amongst themselves. We can also check that $\left[Q^{a}, X^{b}\right]=$ 
$i \epsilon^{a b c} \eta_{c d} X^{d}$ transforms like a vector. This verifies that the Schwarzian realizes the Poincare algebra postulated in (2.9).

One advantage of using the Hamiltonian formalism is that expressions for operators written in terms of $T, T^{\prime}$ and $p_{1}, p_{2}$ are fairly agnostic about what time-evolution rule is being used. For example, we may evolve by $H_{l}+H_{r}$ or the coupled Hamiltonian $H_{l}+H_{r}+$ $\eta\left(X_{l} \cdot X_{r}\right)^{-\Delta}$. This is not the case when we write expressions in the Lagrangian formalism because the expressions for $Q^{a}$ change (for example, they depend on $\eta$ in (4.25)).

As another application of the Hamiltonian formalism, we may check that the approximate construction of the charges discussed in section 4.3 does not lead to a closed algebra. In other words, the failure of the algebra happens not just at the microscopic level (in terms of the fermions) but even in the Schwarzian limit. This is not too surprising because as discussed in [17] and in section 5.1, the spectrum of the eternal traverable wormhole (even in the Schwarzian limit) contains a tensor factor which is not SL(2) invariant.

\section{Evaluating commutators perturbatively}

One might wonder whether we can check the commutator computation using standard perturbation theory. In this section, we show how this may be done. We focus on checking the commutation relations between the gauge charges; the commutation relations between the physical charges $G^{A}$ can be checked quite analogously.

We start by reviewing the linearized Schwarzian action [3]. We use the same variables as in section 4.1. We write the Schwarzian as

$$
I=\frac{\mathfrak{s}}{2} \int d \tilde{u}\left(\epsilon^{\prime \prime 2}+\epsilon^{\prime 2}\right)=\frac{\mathfrak{s}}{2} \int d \tilde{u}\left(-r^{\prime 2}-r^{2}+q^{\prime 2}\right) .
$$

The first line is the same expression as in (4.15), except now in Lorentzian signature. We have rewritten this higher derivative action by introducing the fields $q$ and the ghost field $r$ such that $q=-\epsilon^{\prime \prime}+\epsilon, r=\epsilon^{\prime \prime}$. From this action we can read off the commutation relations

$$
\left[q, q^{\prime}\right]=\frac{i}{\mathfrak{s}}, \quad\left[r, r^{\prime}\right]=-\frac{i}{\mathfrak{s}}
$$

Now as in (4.3), we expand $Q$ in powers of $\epsilon$, and then substitute for $q, r$. To quadratic order, we find

$$
\begin{aligned}
Q^{-1} & \simeq \mathfrak{s}\left(-1-q^{\prime}-\left(r^{2}+r^{\prime} q^{\prime}+r^{2}\right)\right) \\
Q^{+} & \simeq \mathfrak{s}\left(r-r^{\prime}+q\left(r-r^{\prime}\right)+r\left(q^{\prime}-2 r^{\prime}\right)+2 r^{\prime}\left(q^{\prime}+r^{\prime}\right)+2 r^{2}\right) \\
Q^{-} & \simeq \mathfrak{s}\left(-r-r^{\prime}+q\left(r+r^{\prime}\right)+r\left(q^{\prime}+2 r^{\prime}\right)+2 r^{\prime}\left(q^{\prime}+r^{\prime}\right)+2 r^{2}\right) .
\end{aligned}
$$

Then we can verify that $\left[Q^{-1}, Q^{ \pm}\right]= \pm i Q^{ \pm}$and $\left[Q^{+}, Q^{-}\right]=2 i Q^{-1}$ in line with (2.9). To reproduce the linear terms in $Q^{ \pm}$on the r.h.s., it is important to expand to quadratic order the charges in the commutator.

Note that in order to check the algebra at higher orders, one should not only expand $Q$ to higher powers but also modify the commutation relations due to higher order terms in the action. This quickly gets cumbersome; hence the purpose of the previous section. 


\section{Spinor description of the boundary variables}

We have described the boundary motion in terms of a vector $X^{a}$, with $X^{2}=0$. Alternatively, we can use a two component spinor $\lambda_{\alpha}$ and construct $X^{a}$ as

$$
X^{a}=\left(\sigma^{a}\right)^{\alpha \beta} \lambda_{\alpha} \lambda_{\beta},
$$

where $\sigma^{a}$ are Pauli matrices with one index raised, $\left(\sigma^{a}\right)^{\alpha \beta}=\epsilon^{\alpha \gamma}\left(\sigma^{a}\right)_{\gamma}^{\beta}$, and $\sigma^{-1}=$ $\left(\sigma^{-1}\right)_{\gamma}^{\beta}=\hat{\sigma}_{3}, \sigma^{0}=-\hat{\sigma}_{1}$ and $\sigma^{1}=i \hat{\sigma}_{2}$ where $\hat{\sigma}_{i}$ are the three usual Pauli matrices. The inner product between two spinors is antisymmetric $\langle\lambda, \chi\rangle=\epsilon^{\alpha \beta} \lambda_{\alpha} \chi_{\beta}$. This implies that we automatically obey $X^{2}=0$, since we cannot build any non zero $\operatorname{SL}(2)$ invariant from a single $\lambda$. The evolution equation is then

$$
\dot{\lambda}=\not \lambda \lambda, \quad \ddot{\lambda}=(\not R)^{2} \lambda \propto E \lambda
$$

where $\phi=Q_{a} \sigma^{a}$. We could normalize the spinor by a condition of the form $\langle\dot{\lambda}, \lambda\rangle=$ constant so that $\dot{X}^{2}=-1$, this also ensures $Q . X=1$. This gives a simple expression for the evolution. Also, in terms of left and right spinors then the three generators have expressions proportional to

$$
\tilde{P} \propto \frac{\left\langle\lambda_{l}, \phi_{m} \lambda_{r}\right\rangle}{\left\langle\lambda_{l}, \lambda_{r}\right\rangle}, \quad \tilde{E}+\tilde{B} \propto \frac{\left\langle\lambda_{l}, \phi_{m} \lambda_{l}\right\rangle}{\left\langle\lambda_{l}, \lambda_{r}\right\rangle}, \quad \tilde{E}-\tilde{B} \propto \frac{\left\langle\lambda_{r}, \phi_{m} \lambda_{r}\right\rangle}{\left\langle\lambda_{l}, \lambda_{r}\right\rangle}
$$

\section{D.1 Computation of the charges beyond the scrambling time}

As an application of this formalism, we will consider the setup in section (4.2.2). We consider a background solution where $\phi_{r}=\sigma^{3} / 2$ (we have set $\beta=2 \pi$ ) and

$$
\lambda_{r}(0)=(i, i) / \sqrt{2}, \quad \lambda_{l}(0)=(1,-1) / \sqrt{2}
$$

Then, it is easy to find the time evolution, $\lambda_{r}(t)=e^{u \phi_{r}} \lambda_{r}(0)=i\left(e^{u / 2}, e^{-u / 2}\right) / \sqrt{2}$, for example. We want to insert a perturbation. If we inserted the perturbation at zero time, we would expect it to change $Q_{r}$. The charge will be both rotated and rescaled. The rescaling is negligible in the limit $\omega \rightarrow 0$, see (4.21). So the main effect is a small infinitesimal rotation

$$
\phi_{r} \rightarrow \phi_{r}^{\prime}=L Q_{r} L^{-1}, \quad L \sim 1+\vec{\gamma} \vec{\sigma}
$$

where $\gamma$ is a vector of size of order $\omega$. If we insert this same excitation at some early time, then we should conjugate it by the time evolution,

$$
L\left(u_{0}\right)=e^{u_{0} \phi_{r}} L e^{-u_{0} \phi_{r}} \sim 1+2 \hat{\alpha} \sigma_{-}, \quad \sigma_{-}=\left(\begin{array}{ll}
0 & 0 \\
1 & 0
\end{array}\right)
$$

where $\hat{\alpha} \propto \omega e^{-u_{0}}$ and have kept only the terms that are finite in the limit $\omega \rightarrow 0$ and $u_{0} \rightarrow-\infty$ with $\omega e^{-u_{0}}$ fixed. This implies that

$$
\phi_{m}=-\delta \phi_{r}=Q_{r}-L\left(u_{0}\right) Q_{r} L\left(u_{0}\right)^{-1}=-2 \hat{\alpha}\left[\sigma_{-}, \phi_{r}\right]
$$


Now the new value of $\lambda_{r}$ at the origin is just given by

$$
\begin{aligned}
\lambda_{r}^{\prime}(0) & =e^{-u_{0} \phi_{r}^{\prime}} e^{u_{0} \phi_{r}} \lambda_{r}(0)=L\left(u_{0}\right) e^{-u_{0} \phi_{r}} L\left(u_{0}\right)^{-1} e^{u_{0} \phi_{r}} \lambda_{r}(0)= \\
& =L\left(u_{0}\right) \lambda_{r}=\left(1+2 \hat{\alpha} \sigma_{-}\right) \lambda_{r}(0)
\end{aligned}
$$

$\lambda_{l}(0)$ continues to be as in (D.4). Inserting (D.7), (D.4), (D.8) into (D.3) we get (4.22). We also find that

$$
\left\langle\lambda_{l}, \lambda_{r}^{\prime}\right\rangle \propto(1+\hat{\alpha}) \Longrightarrow\left(X_{l} \cdot X_{r}^{\prime}\right) \propto(1+\hat{\alpha})^{2}
$$

\section{E OTOC correlators and the expectation values of charges}

Section 6.2 of [3] considered an OTOC correlator of four operators of dimension $\Delta$. It is also possible to do the same computation for the case that two operators have dimension $\Delta_{1}$ and the other two have dimension $\Delta_{2}$. Then the same method gives the answer

$$
\frac{\left\langle V_{1} W_{3} V_{2} W_{4}\right\rangle}{\left\langle V_{1} V_{2}\right\rangle\left\langle W_{3} W_{4}\right\rangle}=\frac{U\left(2 \Delta_{1}, 1+2 \Delta_{1}-2 \Delta_{2}, \frac{1}{z}\right)}{z^{2 \Delta_{1}}}=\frac{1}{z^{2 \Delta_{1}} \Gamma\left(2 \Delta_{1}\right)} \int_{0}^{\infty} d t e^{-t / z} t^{2 \Delta_{1}-1}(1+t)^{-2 \Delta_{2}}
$$

Here, $z=\mathfrak{s} e^{\tilde{u}} / 8$. Despite appearances this expression is symmetric under $\Delta_{1} \leftrightarrow \Delta_{2}$. We now want to relate this to the computation in section (4.2.2). To evaluate the charges it is necessary to evaluate the distance.

We can extract the distance from (E.1) by taking the limit $\Delta_{2} \rightarrow 0$, since

$$
\frac{1}{\left(X_{3} \cdot X_{4}\right)^{\Delta_{2}}} \sim 1-\Delta_{2} \log \left(X_{3} \cdot X_{4}\right)=1-\Delta_{2} \ell
$$

where $\ell$ is the distance. By going to higher orders in $\Delta_{2}$ we could get higher moments for the distance. Expanding (E.1) to first order in $\Delta_{2}$,

$$
\langle\ell\rangle=2 \frac{1}{z^{2 \Delta_{1}} \Gamma\left(2 \Delta_{1}\right)} \int_{0}^{\infty} d t e^{-t / z} t^{2 \Delta_{1}-1} \log (1+t) .
$$

This has the following expansion for small and large $z$

$$
\langle\ell\rangle \sim 4 \Delta_{1} z, \quad z \ll 1, \quad\langle\ell\rangle \sim 2 \log z, \quad z \gg 1
$$

(E.3) is the general answer. But we are interested in the classical limit, which corresponds to the case where $C \gg 1$. We are also interested in computing this for a state that has definite energy $\omega$.

From classical computations, we expected the distance to have the form

$$
\langle\ell\rangle=2 \log \left[1+2 \Delta_{1} z\right]
$$

But this answer is supposed to hold for states with definite energy $\tilde{E}$. One way to get the energy to be definite is to imagine that $\Delta_{1}$ is large, then we could imagine creating states with definite energy, of order $\Delta_{1}$ times the appropriate redshift factors, which would lie inside $z$. In this case we can do the integral (E.3) by saddle point. If we ignore the 
logarithm, the saddle point and the integral around it gives the gamma function. Again, ignoring the $\log$, the saddle point is at

$$
\frac{1}{z}=\frac{2 \Delta_{1}}{t_{s}} \rightarrow t_{s}=2 \Delta_{1} z
$$

Then we see that we get the expected answer (E.5) by inserting this value of $t_{s}$ into the $\log$ term, $\log (1+t)$, in the integral (E.3).

It is also clear that, to the extent that we can use this saddle point evaluation of this second term, in (E.1), we will get that the distance behaves classically, with the value (E.5). To check whether the saddle point is valid, we can look at the second derivative of the exponent at the saddle which gives

$$
\partial_{t}^{2}\left(-t / z+2 \Delta_{1} \log t\right)=-\frac{2 \Delta_{1}}{t_{s}^{2}}
$$

So we see that the spread around the saddle is $\delta t \sim t_{s} / \sqrt{\Delta_{1}}$ which is always smaller than $t_{s}$, which justifies the saddle point approximation. The conclusion is that the large $\Delta_{1}$ limit of the OTOC computation in [3] reproduces the expression for the distance used in previous sections.

\section{F Stiffer traversable wormholes}

In this section, we consider other alternative to the global energy generator $\tilde{E}$.

In the Maldacena-Qi wormhole, the spectrum at low energies includes both SL(2) excitations and excitations of the boundary. Both the energies are $\sim T^{\prime}$. We would like to modify the eternal wormhole so that the boundary excitations become very heavy. Then we can see the $\mathrm{SL}(2)$ spectrum just by going to low energies.

In the Maldacena-Qi eternal wormhole, one can determine the frequency of oscillation of the boundary graviton by writing down an effective action for the variable $\phi=\log T^{\prime}$ in the Schwarzian approximation. Assuming no bulk matter, the gauge constraints simplify the action so that $\phi$ becomes the coordinate of a non-relativistic particle in a potential $V(\phi)$ :

$$
V(\phi)=e^{2 \phi}-\sum_{\Delta} \eta_{\Delta} e^{2 \Delta \phi}
$$

The first term is from the Schwarzian, the second term is from the Maldacena-Qi interaction. We are considering a slight generalization of their interaction that could arise, if there are matter fields with different dimensions in the bulk, or by imposing more complicated boundary conditions on the matter fields. For the SYK model, we could generate this interaction by not just adding a term $\sim i \eta\left(\sum_{i} \psi_{l}^{i} \psi_{r}^{i}\right)$, but also adding an interaction $\sim \frac{\rho}{N}\left(\sum_{i} \psi_{l}^{i} \psi_{r}^{i}\right)^{2}$. We want to know if the frequency can be made large by a judicious choice of $\eta_{\Delta}$. Note that the validity of this effective potential requires $\eta_{\Delta}<\ll 1$ and $\phi_{\min } \ll 0$. Let us first consider a minimal extension:

$$
V=e^{2 \phi}-\eta e^{2 \Delta \phi}+\rho e^{2 \tilde{\Delta} \phi} .
$$


To simplify the algebra a little, we start with $\tilde{\Delta}=2 \Delta$. Now let us ignore the first term (from the Schwarzian) and see if we can find a solution where this is a valid approximation. We have

$$
\phi_{m}=\frac{1}{2 \Delta} \log (\eta / 2 \rho), \quad T^{\prime}=\left(\frac{\eta}{2 \rho}\right)^{\frac{1}{2 \Delta}}, \quad\left(\frac{\omega}{T^{\prime}}\right)^{2}=2^{\frac{1}{\Delta}} \Delta^{2} \rho\left(\frac{\rho}{\eta}\right)^{\frac{1}{\Delta}-2} .
$$

For some fixed value $0<\Delta<1 / 2$, taking $\eta / \rho \ll 1$ while keeping $\rho$ small and fixed, we can make $\phi_{m} \ll 0$ and make the frequency very large. In order for it to be a good approximation to drop the first term, we need

$$
2^{-1 / \Delta}\left(\frac{\eta}{\rho}\right)^{1 / \Delta} \ll \frac{\eta^{2}}{2 \rho}
$$

Thus in the limit where $\eta / \rho \ll 1$ and $\rho$ is small and held fixed, this condition will be satisfied for $0<\Delta<1 / 2$. In the SYK model, $\tilde{\Delta}=2 / q$ and $\Delta=1 / q$.

Another question we can ask is: how many bound $N_{b}$ states exist for this spectrum? We can compute this in the WKB approximation, with $p=2 N \dot{\phi}=2 N \sqrt{V}$.

$$
\begin{aligned}
N_{b} & =\frac{1}{2 \pi} \oint p d q=\frac{4 N}{2 \pi} \int_{-\infty}^{\phi_{m}} d \phi \sqrt{\eta e^{2 \Delta \phi}-\rho e^{4 \Delta \phi}} \\
& =\frac{2 N}{\pi}\left(\frac{\eta^{2}}{2 \rho}\right)^{1 / 2} \int_{-\infty}^{0} d \phi \sqrt{e^{2 \Delta \phi}-\frac{1}{2} e^{4 \Delta \phi}} \\
& =\left(\frac{N \rho^{1 / 2}}{\pi}\right)\left(\frac{\eta}{\rho}\right) \frac{2+\pi}{4 \Delta}
\end{aligned}
$$

So we see that the number of states is large in the classical limit, although it is suppressed now by $\eta / \rho^{1 / 2}$. So we need this quantity to be small but not too small if we want the classical analysis to be correct.

Now if we add some matter in the bulk with energy $E_{\text {bulk }}$, then should set the gauge charges of the Schwarzian mode $Q_{0}=E_{\text {bulk }}$. This changes the effective potential by an amount

$$
V \rightarrow V+E_{\mathrm{bulk}} e^{\phi} / N
$$

Notice that for $\Delta<1 / 4$, there is a significant difference between our model and the Malda+Qi wormhole. In particular, suppose we add $N$ particles of energy $\Delta$. In the regime of interest, neither $T^{\prime}$ nor the frequency will change appreciably! This is completely different from the Maldacena-Qi wormhole, where the $e^{\phi}$ term will typically dominate over the $e^{2 \phi}$ term in the potential and change both $T^{\prime}$ and $\omega$.

It is interesting to compute the ground state energy in the presence of matter $q_{0}$ : is the non-linear term in $q_{0}$ suppressed? The first order correction is $T^{\prime} E_{\mathrm{bulk}} / N$. The second order correction is obtained by correcting $\delta \phi=-T_{*}^{\prime} E /\left(N V^{\prime \prime}\right)$. Defining the frequency $\omega^{2}=V_{*}^{\prime \prime} / T^{2}$ :

$$
N \delta V=E T_{*}^{\prime}-\frac{1}{2 N} \frac{E^{2}}{\omega^{2}}+O\left(\frac{1}{N^{2}}\right)
$$



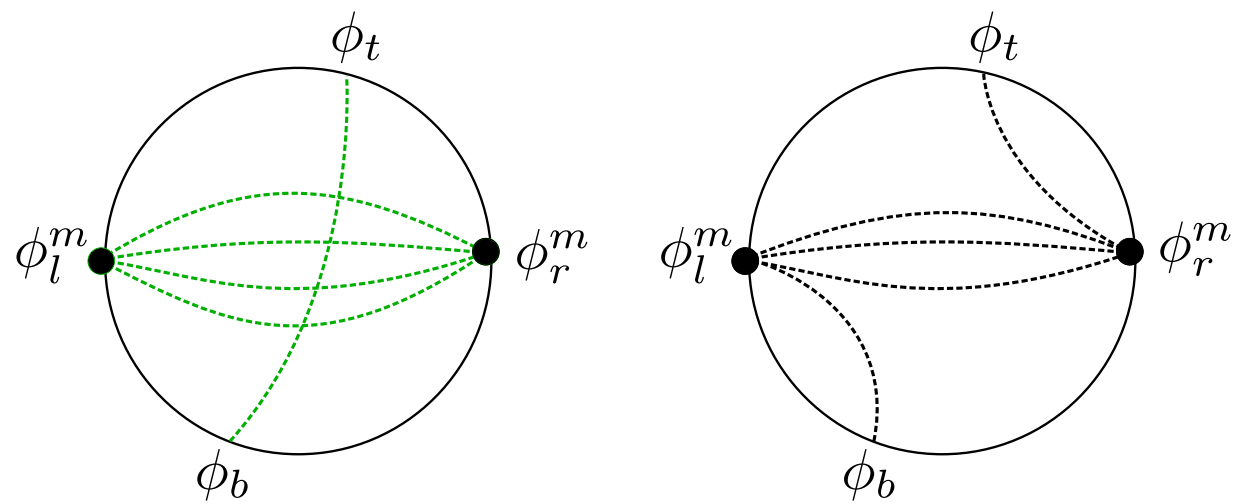

Figure 12. We consider the diagrams contributing to the correlator $\left\langle\phi\left(u_{t}\right)\left|\phi_{l}^{m} \phi_{r}^{m}\right| \phi\left(u_{b}\right)\right\rangle$ in (G.1). In green is the desired contribution which mimics the action of the charges in figure $5(\mathrm{a})$. The "bad" diagrams (displayed in black) are morally similar to the ones in figure 5(b).

This comes from both the contribution of the original potential and the matter piece. We see that when $\omega$ is large, the correction is suppressed.

It would be interesting to analyze this model further, e.g., explore its thermodynamics, study it at higher temperatures in large- $q$ SYK, etc. We leave this to future work.

\section{G Measuring distance using a single free field}

We suspect that the distance could be measured even if we have a small number of fields in the bulk. As a simple example, consider a single free bosonic field in the bulk with dimension $\Delta$. Then we may measure the distance using considering the operator $d \Delta \sim$ $-\log \phi_{l} \phi_{r}$. This operator can be defined by the replica trick, e.g., we consider higher-pt correlation functions and then continue to $m=0$.

Now we would like to see how well this operator tracks the distance when there are $\phi$ particles around. We can consider the states defined previously in section 4.2,

$$
\left\langle\phi\left(u_{t}\right)\left|\phi_{l}^{m} \phi_{r}^{m}\right| \phi\left(u_{b}\right)\right\rangle=m ! G_{l r}^{m} G_{t b}+2 m^{2}(m-1) ! G_{l r}^{m-1}\left(G_{l t} G_{r b}+G_{r t} G_{l b}\right) .
$$

Here the first term comes from the "good" diagrams in figure 12; the "bad" diagrams ones are contractions where one of the $\phi_{l}$ contract with the matter created from $\phi_{b}$ or $\phi_{t}$. The factor of 2 comes from the choice of connecting one of the $\phi_{l}$ to $\phi_{t}$ or to $\phi_{b}$. The operators $\phi^{m}$ should be regulated, for instance by point-splitting. Analytically continuing,

$$
\begin{aligned}
d \Delta & \sim-\lim _{m \rightarrow 0} \frac{1}{G_{t b}}\left\langle\phi\left(u_{t}\right)\left|\left(\frac{\left(\phi_{l} \phi_{r}\right)^{m}-1}{m}\right)\right| \phi\left(u_{b}\right)\right\rangle \\
& =-\log \left(G_{l r}\right)+\gamma-2 \frac{G_{l t} G_{r b}+G_{l b} G_{r t}}{G_{l r} G_{t b}} .
\end{aligned}
$$

In the limit of large distances (e.g., when we push the bulk fields towards the boundary) or when $\Delta$ is large, the leading contribution is the first term, which is precisely the distance. 
As a generalization of this calculation, ${ }^{25}$ we may consider inserting not just a single particle but several particles, e.g., $\phi_{b} \rightarrow \phi_{b}^{k}, \phi_{t} \rightarrow \phi_{t}^{k}$. The analog of (G.2) will involve a sum over the number of bonds broken between the left and right. This will generate terms that depend on $k$. The $k$-dependence of the "bad" terms means that there is likely a bound on how many particles we can insert before the operator defined above no longer tracks the distance. This is not too surprising, for example, if we considered some coherent state with a large number of quanta, we expect that the expectation value of $\log \phi_{l} \phi_{r}$ is determined by the classical field values, which does not have to track the distance.

The preliminary conclusion is that at least for small number of quanta, the operator $-\log \left(\phi_{l} \phi_{r}\right)$ seems to be a good proxy for the distance between the two sides. We leave to future work a more thorough understanding of the limitations of this distance operator, the effects of interactions, etc.

Open Access. This article is distributed under the terms of the Creative Commons Attribution License (CC-BY 4.0), which permits any use, distribution and reproduction in any medium, provided the original author(s) and source are credited.

\section{References}

[1] A. Almheiri and J. Polchinski, Models of AdS 2 backreaction and holography, JHEP 11 (2015) 014 [arXiv: 1402.6334] [INSPIRE].

[2] K. Jensen, Chaos in AdS 2 holography, Phys. Rev. Lett. 117 (2016) 111601 [arXiv: 1605.06098] [INSPIRE].

[3] J. Maldacena, D. Stanford and Z. Yang, Conformal symmetry and its breaking in two dimensional nearly anti-de-Sitter space, PTEP 2016 (2016) 12C104 [arXiv:1606.01857] [INSPIRE].

[4] J. Engelsöy, T.G. Mertens and H. Verlinde, An investigation of $A d S_{2}$ backreaction and holography, JHEP 07 (2016) 139 [arXiv: 1606. 03438] [INSPIRE].

[5] S. Sachdev and J.-W. Ye, Gapless spin fluid ground state in a random, quantum Heisenberg magnet, Phys. Rev. Lett. 70 (1993) 3339 [cond-mat/9212030] [INSPIRE].

[6] A. Kitaev, A simple model of quantum holography (part 1, talk at KITP, http://online.kitp.ucsb.edu/online/entangled15/kitaev/, University of California, Santa Barbara, CA, U.S.A., 7 April 2015.

[7] A. Kitaev, A simple model of quantum holography (part 2, talk at KITP, http://online.kitp.ucsb.edu/online/entangled15/kitaev2/, University of California, Santa Barbara, CA, U.S.A., 27 May 2015.

[8] A. Kitaev and S.J. Suh, The soft mode in the Sachdev-Ye-Kitaev model and its gravity dual, JHEP 05 (2018) 183 [arXiv: 1711. 08467] [INSPIRE].

[9] J. Maldacena and D. Stanford, Remarks on the Sachdev-Ye-Kitaev model, Phys. Rev. D 94 (2016) 106002 [arXiv: 1604.07818] [INSPIRE].

[10] A. Kitaev and S.J. Suh, Statistical mechanics of a two-dimensional black hole, JHEP 05 (2019) 198 [arXiv: 1808.07032] [INSPIRE].

\footnotetext{
${ }^{25}$ We thank Wayne Zhao for discussions about the combinatorics.
} 
[11] Z. Yang, The quantum gravity dynamics of near extremal black holes, JHEP 05 (2019) 205 [arXiv: 1809.08647] [INSPIRE].

[12] D. Bagrets, A. Altland and A. Kamenev, Sachdev-Ye-Kitaev model as Liouville quantum mechanics, Nucl. Phys. B 911 (2016) 191 [arXiv:1607.00694] [InSPIRE].

[13] D. Bagrets, A. Altland and A. Kamenev, Power-law out of time order correlation functions in the SYK model, Nucl. Phys. B 921 (2017) 727 [arXiv:1702.08902] [INSPIRE].

[14] D. Stanford and E. Witten, Fermionic localization of the Schwarzian theory, JHEP 10 (2017) 008 [arXiv: 1703.04612] [INSPIRE].

[15] T.G. Mertens, G.J. Turiaci and H.L. Verlinde, Solving the Schwarzian via the conformal bootstrap, JHEP 08 (2017) 136 [arXiv:1705.08408] [INSPIRE].

[16] P. Gao, D.L. Jafferis and A. Wall, Traversable wormholes via a double trace deformation, JHEP 12 (2017) 151 [arXiv: 1608.05687] [INSPIRE].

[17] J. Maldacena and X.-L. Qi, Eternal traversable wormhole, arXiv:1804.00491 [INSPIRE].

[18] L. Susskind, Why do things fall?, arXiv:1802.01198 [INSPIRE].

[19] A.R. Brown, H. Gharibyan, A. Streicher, L. Susskind, L. Thorlacius and Y. Zhao, Falling toward charged black holes, Phys. Rev. D 98 (2018) 126016 [arXiv:1804.04156] [InSPIRE].

[20] X.-L. Qi and A. Streicher, Quantum epidemiology: operator growth, thermal effects and SYK, arXiv: 1810.11958 [INSPIRE].

[21] D.A. Roberts, D. Stanford and A. Streicher, Operator growth in the SYK model, JHEP 06 (2018) 122 [arXiv : 1802.02633] [INSPIRE].

[22] R. Jackiw, Lower dimensional gravity, Nucl. Phys. B 252 (1985) 343 [INSPIRE].

[23] C. Teitelboim, Gravitation and Hamiltonian structure in two space-time dimensions, Phys. Lett. B 126 (1983) 41 [INSPIRE].

[24] M. Henneaux, Quantum gravity in two-dimensions: exact solution of the Jackiw model, Phys. Rev. Lett. 54 (1985) 959 [INSPIRE].

[25] D. Louis-Martinez, J. Gegenberg and G. Kunstatter, Exact Dirac quantization of all $2 D$ dilaton gravity theories, Phys. Lett. B 321 (1994) 193 [gr-qc/9309018] [INSPIRE].

[26] P. Saad, S.H. Shenker and D. Stanford, JT gravity as a matrix integral, arXiv:1903.11115 [INSPIRE].

[27] Y. Sekino and L. Susskind, Fast scramblers, JHEP 10 (2008) 065 [arXiv:0808.2096] [INSPIRE].

[28] K.V. Kuchar, Geometrodynamics of Schwarzschild black holes, Phys. Rev. D 50 (1994) 3961 [gr-qc/9403003] [INSPIRE].

[29] D. Harlow and D. Jafferis, The factorization problem in Jackiw-Teitelboim gravity, arXiv: 1804.01081 [INSPIRE].

[30] S.H. Shenker and D. Stanford, Black holes and the butterfly effect, JHEP 03 (2014) 067 [arXiv:1306.0622] [INSPIRE].

[31] A. Kitaev, Hidden correlations in the Hawking radiation and thermal noise, talk given at the Fundamental Physics Prize Symposium, https://www.youtube.com/watch?v=OQ9qN8j7EZI, U.S.A., 10 November 2014. 
[32] J. Maldacena, D. Stanford and Z. Yang, Diving into traversable wormholes, Fortsch. Phys. 65 (2017) 1700034 [arXiv: 1704.05333] [INSPIRE].

[33] A. Blommaert, T.G. Mertens and H. Verschelde, Clocks and rods in Jackiw-Teitelboim quantum gravity, arXiv:1902.11194 [INSPIRE].

[34] S.R. Coleman and F. De Luccia, Gravitational effects on and of vacuum decay, Phys. Rev. D 21 (1980) 3305 [INSPIRE].

[35] M. Dafermos and J. Luk, The interior of dynamical vacuum black holes I: the $C^{0}$-stability of the Kerr Cauchy horizon, arXiv:1710.01722 [INSPIRE].

[36] J. Maldacena, Vacuum decay into anti de Sitter space, arXiv:1012.0274 [INSPIRE].

[37] A. Hamilton, D.N. Kabat, G. Lifschytz and D.A. Lowe, Local bulk operators in AdS/CFT: a boundary view of horizons and locality, Phys. Rev. D 73 (2006) 086003 [hep-th/0506118] [INSPIRE].

[38] I. Heemskerk, D. Marolf, J. Polchinski and J. Sully, Bulk and transhorizon measurements in AdS/CFT, JHEP 10 (2012) 165 [arXiv:1201.3664] [INSPIRE].

[39] D. Kabat, G. Lifschytz and D.A. Lowe, Constructing local bulk observables in interacting AdS/CFT, Phys. Rev. D 83 (2011) 106009 [arXiv:1102.2910] [INSPIRE].

[40] D.L. Jafferis, Bulk reconstruction and the Hartle-Hawking wavefunction, arXiv:1703.01519 [INSPIRE].

[41] I. Heemskerk, Construction of bulk fields with gauge redundancy, JHEP 09 (2012) 106 [arXiv:1201.3666] [INSPIRE].

[42] D. Kabat and G. Lifschytz, Decoding the hologram: scalar fields interacting with gravity, Phys. Rev. D 89 (2014) 066010 [arXiv:1311.3020] [INSPIRE].

[43] A. Streicher, private communication.

[44] D.J. Gross and V. Rosenhaus, The bulk dual of SYK: cubic couplings, JHEP 05 (2017) 092 [arXiv: 1702.08016] [INSPIRE].

[45] L. Susskind, Complexity and Newton's laws, arXiv:1904.12819 [INSPIRE].

[46] D. Stanford and L. Susskind, Complexity and shock wave geometries, Phys. Rev. D 90 (2014) 126007 [arXiv: 1406 . 2678] [INSPIRE].

[47] H. Casini, M. Huerta and R.C. Myers, Towards a derivation of holographic entanglement entropy, JHEP 05 (2011) 036 [arXiv:1102.0440] [INSPIRE].

[48] W. Donnelly, Entanglement entropy and non-Abelian gauge symmetry, Class. Quant. Grav. 31 (2014) 214003 [arXiv: 1406.7304] [INSPIRE].

[49] L. Susskind, Computational complexity and black hole horizons, Fortsch. Phys. 64 (2016) 44 [Addendum ibid. 64 (2016) 44] [arXiv:1402.5674] [arXiv:1403.5695] [INSPIRE].

[50] A.R. Brown, H. Gharibyan, H.W. Lin, L. Susskind, L. Thorlacius and Y. Zhao, Complexity of Jackiw-Teitelboim gravity, Phys. Rev. D 99 (2019) 046016 [arXiv:1810.08741] [INSPIRE].

[51] L. Susskind, Dear qubitzers, GR=QM, arXiv:1708.03040 [INSPIRE].

[52] D. Harlow, Wormholes, emergent gauge fields and the weak gravity conjecture, JHEP 01 (2016) 122 [arXiv:1510.07911] [INSPIRE]. 